\title{
CAHN-HILLIARD-NAVIER-STOKES SYSTEMS WITH MOVING CONTACT LINES
}

\author{
C.G. GAL, M. GRASSELLI, AND A. MIRANVILLE
}

\begin{abstract}
We consider a well-known diffuse interface model for the study of the evolution of an incompressible binary fluid flow in a two or three-dimensional bounded domain. This model consists of a system of two evolution equations, namely, the incompressible Navier-Stokes equations for the average fluid velocity $u$ coupled with a convective Cahn-Hilliard equation for an order parameter $\phi$. The novelty is that the system is endowed with boundary conditions which account for a moving contact line slip velocity. The existence of a suitable global energy solution is proven and the convergence of any such solution to a single equilibrium is also established.
\end{abstract}

\section{INTRODUCTION}

The motion of an isothermal mixture of two immiscible and incompressible fluids subject to phase separation can be described by a well-known diffuse interface model: the Navier-Stokes equations for the average fluid velocity $u$ are nonlinearly coupled with the convective CahnHilliard equation for the order parameter $\phi$. The latter represents the difference of the relative concentrations of the two fluids. This model is called model $\mathrm{H}$, but it is also known as CahnHilliard-Navier-Stokes (CHNS) system (see, e.g., [5, 36, 40, 37, 38, 47, 61], cf. also [13, 18, 42, 50, 65])

A simplified version of the CHNS system is the following

$$
\begin{aligned}
& \partial_{t} u+u \cdot \nabla u-\operatorname{div}(2 \nu D(u))+\nabla p=\varepsilon \mu \nabla \phi+h, \\
& \operatorname{div}(u)=0, \\
& \partial_{t} \phi+u \cdot \nabla \phi-\varrho_{0} \Delta \mu=0, \mu=-\epsilon \Delta \phi+\varepsilon^{-1} f(\phi),
\end{aligned}
$$

in $\Omega \times(0, \infty)$. Here, $\Omega$ is a bounded domain in $\mathbb{R}^{\mathrm{N}}, \mathrm{N}=2,3$, with a sufficiently smooth boundary $\Gamma$ (say, of class $\mathcal{C}^{2}$ at least) and $h=h(t)$ is an external body force. We consider the model with matched densities and suppose the density equal to one. The quantities $\nu, \varrho_{0}$ denote the kinematic viscosity of the fluid and the mobility of the mixture, both supposed to be constants, while $\varepsilon>0$ is related to the thickness of the interface separating the two fluids. As usual, $D(u)$ denotes the deformation tensor, i.e., $[D(u)]_{i, j}=\frac{1}{2}\left(\partial_{i} u_{j}+\partial_{j} u_{i}\right), 1 \leq i, j \leq N$. Also, we recall that the term $\mu \nabla \phi$ is known as Korteweg force and $\mu$ is the so-called chemical potential. The latter is the variational derivative of the Helmholtz free energy functional

$$
\mathcal{F}(\phi)=\int_{\Omega}\left(\frac{\varepsilon}{2}|\nabla \phi|^{2}+\varepsilon^{-1} F(\phi)\right) d x,
$$

where $F(r)=\int_{0}^{r} f(\zeta) d \zeta$ is the potential density. A physically relevant example of $F$ is the so-called logarithmic potential, namely,

$$
F(s)=c_{0}[(1+s) \ln (1+s)+(1-s) \ln (1-s)]-c_{1} s^{2}, \quad c_{1}>c_{0}>0,
$$

for $s \in[-1,1]$. This potential is very often replaced by a double well polynomial approximation, such as $F(s)=\gamma_{1} s^{4}-\gamma_{2} s^{2}, s \in \mathbb{R}$, where $\gamma_{1}$ and $\gamma_{2}$ are given positive constants.

There are many mathematical results on the system (1.1)-(1.4). They have been proven under various assumptions on the domain $\Omega$, on the coefficients as well as on $F$. Confining ourselves to the ones related to model $\mathrm{H}$, we mention, e.g., $[1,2,3,8,9,10,11,12,15,14,16,28,29,30,31$, $39,67,76,78]$ (see also $[6,23,24,41,43,45,46,49,62,68]$ for the numerical approximation and simulations). Nevertheless, the boundary conditions taken into consideration so far are rather

2010 Mathematics Subject Classification. 35K55, 35Q35, 76D05.

Key words and phrases. Navier-Stokes equations, incompressible fluids, Cahn-Hilliard equations, dynamic boundary conditions, Navier boundary conditions, existence, convergence to equilibria. 
standard. More precisely, in almost all the contributions, $u: \Omega \rightarrow \mathbb{R}^{\mathrm{N}}$ is subject to a non-slip or periodic boundary conditions, while $\phi: \Omega \rightarrow \mathbb{R}$ and $\mu: \Omega \rightarrow \mathbb{R}$ are subject to homogeneous Neumann boundary conditions or periodic boundary conditions in the shear case. The nonslip boundary condition for $u$ is quite common in the literature on Navier-Stokes equations. Nevertheless, a more realistic partial slip can be described through the so-called Navier boundary condition (see [55]), namely, $2 \nu(D(u) \cdot n)_{\tau}+\beta u_{\tau}=0$, where $u_{\tau}$ is the (tangential) slip velocity at the boundary $\Gamma$ measured relatively to the wall, with $\beta>0$ being a slip coefficient. We recall that this condition was also derived by Maxwell [52] in the kinetic theory of gases.

Consider now an immiscible two-phase incompressible flow where one fluid displaces the other along the boundary $\Gamma$. It was observed (see, e.g., [21]) that the moving contact line (MCL), defined as the intersection of the fluid-fluid interface with the solid wall, is incompatible with the non-slip boundary condition (cf. [22, 54] and their references). As shown in [22], under the usual hydrodynamic assumptions, namely, incompressible Newtonian fluids, non-slip boundary condition and smooth rigid walls, there is a velocity discontinuity at the moving contact line, and the tangential force exerted by the fluids on the solid surface $\Gamma$ in the vicinity of the contact line becomes infinite. Thus, in immiscible two-phase flows, none of the standard boundary conditions can account for the moving contact line slip velocity profiles obtained from simulations and, therefore, new boundary conditions were required to describe the observed phenomena. In order to account for moving contact lines a generalization of the Navier boundary conditions has been proposed in [59] (see also [58]) using the laws of thermodynamics and variational principles related to the minimum energy dissipation. These laws state that the entropy associated with the composition diffusion and the work done by the flow to the fluid-fluid interface are conserved. We refer the reader to the appendix for more details. As a consequence, one deduces the following generalized Navier boundary conditions (GNBC)

$$
\begin{aligned}
& u \cdot n=0, \partial_{n} \mu=0, \\
& 2 \nu(D(u) \cdot n)_{\tau}+\beta u_{\tau}=\mathcal{L}(\phi) \nabla_{\tau} \phi, \\
& \partial_{t} \phi+u_{\tau} \cdot \nabla_{\tau} \phi=-l_{0} \mathcal{L}(\phi),
\end{aligned}
$$

on $\Gamma \times(0, \infty)$, where

$$
\mathcal{L}(\phi):=-\gamma \Delta_{\tau} \phi+\varepsilon \partial_{n} \phi+\zeta \phi+g(\phi) .
$$

Here $\nabla_{\tau}$ denotes the tangential gradient operator defined along the tangential direction $\tau=$ $\left(\tau_{1}, \ldots, \tau_{N-1}\right)$ at $\Gamma$ and $\Delta_{\tau}$ denotes the Laplace-Beltrami operator on $\Gamma$. Moreover, $l_{0}, \beta, \zeta, \gamma>0$ are four phenomenological parameters with $\beta$ being a slip coefficient. In general, if $n$ denotes the exterior unit normal vector to $\Gamma$, then, for any vector $v: \Gamma \rightarrow \mathbb{R}^{\mathrm{N}}, v_{n}=v \cdot n$ is the normal component of the vector field, while $v_{\tau}=v-\left(v_{n}\right) n$ corresponds to the tangential component of $v$. The function $g$ in (1.9) is a nonlinear function of the local composition which accounts for the interfacial energy at the mixture-wall interface.

We emphasize that (1.6) together with (1.2) also ensure mass conservation. Indeed, the following quantity

$$
\langle\phi(t)\rangle:=|\Omega|^{-1} \int_{\Omega} \phi(x, t) d x
$$

is conserved for all time. More precisely, one easily deduces from (1.3) that $\langle\phi(t)\rangle=\langle\phi(0)\rangle$, for all $t \geq 0$. System (1.1)-(1.9) is also subject to the initial conditions

$$
u_{\mid t=0}=u_{0}, \quad \phi_{\mid t=0}=\phi_{0}, \quad \text { in } \Omega .
$$

As far as we know, the existence of a (weak) solution to problem (1.1)-(1.10) has not yet been proven, though there are some papers devoted to its numerical approximation (see, e.g., $[7,33,63,72]$ and cf. also [73, 75] for the formal interface limit). Here the main goal is to establish the existence of a global weak solution (of finite energy) to problem (1.1)-(1.10) when $\gamma>0$. This means that we assume some surface diffusion on $\Gamma$; we can note that the dynamic boundary conditions proposed (for the sole Cahn-Hilliard equation) in order to account for the dynamic interactions with the walls consider indeed such a surface diffusion, see [26, 27, 44]. On the other hand, we recall that surface diffusion is not considered in [59] (i.e. $\gamma=0$ ). In addition, we prove that any weak energy solution converges to a single equilibrium. The key step is a novel approximation scheme which relies rather on spatial mollifiers and some sharp inequalities of Poincaré-Young type to exploit fixed point like arguments. Indeed we are not able 
to implement here Galerkin type approximations since the test functions needed to derive the energy inequality are not compatible with the truncations that we have to introduce (see Remark 3.1 below). We also mention that the presence of the Young stress $\mathcal{L}(\phi) \nabla_{\tau} \phi$ at the boundary wall and the boundary advection term entail a strong coupling of system (1.1)-(1.3) through the boundary conditions (1.7)-(1.8), but only weakly coupled through the interfacial force $\mu \nabla \phi$ in the bulk equation (1.1). The latter is sufficiently weak in the inviscid case as well (see [16]). These features will be also reflected in the statements of our main theorems. In particular, we note that the strong coupling at the boundary does not allow us to obtain the uniqueness of an energy solution even in two dimensions.

The plan goes as follows. The functional framework is detailed in Section 2, where the weak formulation is introduced. Section 3 contains the statements of the main results. In Section 4 we report a number of technical tools and known results which are essential to carry out the proofs. Existence is proven in Section 5 for regular potentials, while the corresponding convergence result to equilibria is proven in Section 6. Section 7 deals with the case of a singular (e.g., logarithmic) potential. An appendix is devoted to the derivation of the model.

\section{THE FUnCTIONAL FRAMEWORK}

Without loss of generality, we set $l_{0}, \varrho_{0}$ and $\varepsilon$ equal to one. We denote by $\|\cdot\|_{p}$ and $\|\cdot\|_{p, \Gamma}$ the norms on $L^{p}(\Omega)$ and $L^{p}(\Gamma)$, respectively. In the case $p=2,(\cdot, \cdot)\left(\right.$ or $\left.(\cdot, \cdot)_{\Gamma}\right)$ stands for the usual scalar product which induces the $L^{2}(\Omega)$-norm (or the $L^{2}(\Gamma)$-norm). The norms on $H^{s}(\Omega)$ and $H^{s}(\Gamma)$ are indicated by $\|\cdot\|_{H^{s}}$ and $\|\cdot\|_{H^{s}(\Gamma)}$, respectively, for any $s \in \mathbb{R}$. Next, recall that the Dirichlet trace map $\operatorname{tr}_{\mathrm{D}}:\left\{\left.\phi\right|_{\bar{\Omega}}: \phi \in C^{\infty}\left(\mathbb{R}^{N}\right)\right\} \rightarrow C^{\infty}(\Gamma)$, defined by $\operatorname{tr}_{\mathrm{D}}(\phi)=\phi_{\mid \Gamma}$, extends to a linear continuous operator $\operatorname{tr}_{\mathrm{D}}: H^{r}(\Omega) \rightarrow H^{r-1 / 2}(\Gamma)$, for all $r>1 / 2$, which is onto for $1 / 2<r<3 / 2$. On account of the boundary condition (1.8), we also need to introduce the functional spaces

$$
V_{\gamma}^{s}=\left\{(\phi, \psi) \in H^{s}(\Omega) \times H^{s-1 / 2}(\Gamma): \psi=\operatorname{tr}_{\mathrm{D}}(\phi) \in H^{s}(\Gamma)\right\}, \text { if } \gamma>0,
$$

where $s \geq 0$, equipped with norms $\|\cdot\|_{V_{\gamma}^{s}}$ defined as follows:

$$
\|(\phi, \psi)\|_{V_{\gamma}^{s}}^{2}=\|\phi\|_{H^{s}}^{2}+\gamma\|\psi\|_{H^{s}(\Gamma)}^{2} .
$$

In particular, when $s=0,1$, the corresponding (equivalent) norms $\|\cdot\|_{V_{\gamma}^{s}}$ are given by

for some $\zeta>0$, and

$$
\|(\phi, \psi)\|_{V_{\gamma}^{1}}^{2}=\int_{\Omega}|\nabla \phi|^{2} d x+\int_{\Gamma}\left(\gamma\left|\nabla_{\tau} \psi\right|^{2}+\zeta|\psi|^{2}\right) d S
$$

$$
\|(\phi, \psi)\|_{V_{\gamma}^{0}}^{2}=\int_{\Omega}|\phi|^{2} d x+\int_{\Gamma} \zeta|\psi|^{2} d S,
$$

respectively. Furthermore, we notice that $V_{\gamma}^{s}$ is compactly embedded in $V_{\gamma}^{s-1}$, for all $s \geq 1$ and $\gamma>0$. We also recall the following continuous embeddings: $H^{1}(\Gamma) \subset L^{\infty}(\Gamma)$ if $N=2$ and $H^{1 / 2}(\Gamma) \subset L^{s}(\Gamma)$, for any fixed $s \in[1, \infty)$, if $N=2$, with $s=4$ if $N=3$, and $H^{1}(\Gamma) \hookrightarrow L^{q}(\Gamma)$, for every $q \in[1, \infty)$, if $N=3$. Moreover, the symbol $\langle\cdot, \cdot\rangle$ stands for the duality pairing between any (real) Banach space $X$ and its dual $X^{*}$.

We now recall the corresponding framework associated with the Cahn-Hilliard system (1.3), (1.8)-(1.9). It is well known that the Laplace-Beltrami operator $A_{\tau}:=-\Delta_{\tau}$ is a nonnegative self-adjoint operator in $L^{2}(\Gamma)$. Thus the Sobolev spaces $H^{s}(\Gamma), s \in \mathbb{R}$, can also be defined as $H^{s}(\Gamma)=D\left(\left(A_{\tau}+I\right)^{s / 2}\right)$. In particular, $\|\psi\|_{2, \Gamma}+\left\|A_{\tau} \psi\right\|_{2, \Gamma}$ is an equivalent norm on $H^{2}(\Gamma)$ and $\|\psi\|_{2, \Gamma}+\left\|\nabla_{\tau} \psi\right\|_{2, \Gamma}$ is an equivalent norm on $H^{1}(\Gamma)$. Next, we introduce the operator

$$
A_{N} \phi=-\Delta \phi, \text { for } \phi \in D\left(A_{N}\right)=\left\{\phi \in H^{2}(\Omega): \partial_{n} \phi=0 \text { on } \Gamma\right\},
$$

and we endow $D\left(A_{N}\right)$ with the norm $\left\|A_{N}(\cdot)\right\|_{2}+|\langle\cdot\rangle|$, where $\langle u\rangle=|\Omega|^{-1}\langle u, 1\rangle$. This norm is equivalent to the $H^{2}(\Omega)$-norm. Also, we define the linear positive unbounded operator on the Hilbert space $L_{0}^{2}(\Omega)$ of the $L^{2}$-functions with null mean

$$
B_{N} \phi=-\Delta \phi, \quad \text { for } \phi \in D\left(B_{N}\right)=D\left(A_{N}\right) \cap L_{0}^{2}(\Omega) .
$$

Observe that $B_{N}^{-1}$ is a compact linear operator on $L_{0}^{2}(\Omega)$. More generally, we can define $B_{N}^{s}$ for any $s \in \mathbb{R} \backslash\{ \pm 3 / 4\}$, noting that $\left\|B_{N}^{s / 2}(\cdot)\right\|_{2}$ is an equivalent norm in $H^{s}(\Omega)$, where $H^{s}(\Omega):=$ 
$\left(H^{-s}(\Omega)\right)^{*}$, whenever $s<0$. Note that $A_{N} \equiv B_{N}$ on $D\left(B_{N}\right)$ and if $\phi$ is such that $\phi-\langle\phi\rangle \in$ $D\left(B_{N}^{s / 2}\right)$, then $\left\|B_{N}^{s / 2}(\phi-\langle\phi\rangle)\right\|_{2}+|\langle\phi\rangle|$ is equivalent to the $H^{s}(\Omega)$-norm whenever $s \in \mathbb{R} \backslash\{ \pm 3 / 4\}$.

We now introduce the functional framework associated with the equation for the fluid velocity. To this end, we consider a (real) Hilbert space $X$ and denote by $\mathbb{X}$ the space $X \times \ldots \times X(N$-times), endowed with the product structure, and by $\mathbb{X}^{*}$ its dual; $\|\cdot\|_{\mathbb{X}^{*}}$ will denote the dual norm of $\|\cdot\|$ on $\mathbb{X}^{*}$. Then we introduce the spaces $\mathbb{H}=\mathbb{H}^{0}$ and $\mathbb{H}^{s}, s \geq 1$, defined by

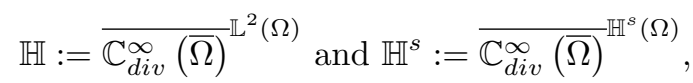

where

$$
\mathbb{C}_{\text {div }}^{\infty}(\bar{\Omega})=\left\{u \in \mathbb{C}^{\infty}(\bar{\Omega}): \nabla \cdot u=0 \text { in } \Omega, u \cdot n=0 \text { on } \Gamma\right\} .
$$

The space $\mathbb{H}$ is endowed with the scalar product and the norm of $\mathbb{L}^{2}(\Omega)$, denoted by $(\cdot, \cdot)$ and $|\cdot|$, respectively. In order to handle the boundary conditions (1.6)-(1.7), we introduce the bilinear form $a(\cdot, \cdot)$ on $\mathbb{H}^{1} \times \mathbb{H}^{1}$ as follows:

$$
a_{0}(u, v)=2 \nu(D(u), D(v))+\beta\left(u_{\tau}, v_{\tau}\right)_{\Gamma}, \beta>0,
$$

for all $u, v \in \mathbb{H}^{1}$. We recall that $a_{0}(\cdot, \cdot)$ is a coercive, continuous, symmetric and bilinear form on the space $\mathbb{H}^{1}$ and that $\sqrt{a_{0}(u, u)}$ is equivalent to the $\mathbb{H}^{1}$-norm (which we denote by $\|\cdot\|$ ) for vectors that belong to $\mathbb{H}^{1}$. The corresponding Stokes operator $A_{0}$ associated with the bilinear form $a_{0}$ is given by $A_{0} u=-P \operatorname{div}(2 \nu D(u))$ such that $\left(A_{0} u, v\right)=a_{0}(u, v)$, for all

$$
u \in D\left(A_{0}\right)=\left\{u \in \mathbb{H}^{2}: 2 \nu(D(u) \cdot n)_{\tau}+\beta u_{\tau}=0 \text { a.e. on } \Gamma\right\} .
$$

Here $P: \mathbb{L}^{2}(\Omega) \rightarrow \mathbb{H}$ denotes the Helmholtz (orthogonal) projector onto $\mathbb{H}$. It follows that we also have the following compact embeddings $D\left(A_{0}\right) \subset \mathbb{H}^{1}=D\left(A_{0}^{1 / 2}\right) \subset \mathbb{H}$ and the operator $A_{0}$ is positive and selfadjoint on $\mathbb{H}$, and $A_{0}^{-1}$ is compact (see, for instance, [2, Appendix] and [31, Section 2]).

In order to define the weak formulation of (1.1)-(1.10), we also need to introduce the bilinear operators $B_{0}, B_{1}, B_{\Gamma}$ (and their related trilinear forms $b_{0}, b_{1}$ and $b_{\Gamma}$ ). More precisely, we set

$$
b_{0}(u, v, w):=\left(B_{0}(u, v), w\right)=\int_{\Omega}[(u \cdot \nabla) v] \cdot w d x,
$$

for $u, v, w \in C^{\infty}\left(\bar{\Omega}, \mathbb{R}^{\mathrm{N}}\right)$, and

$$
b_{1}(u, \phi, \psi):=\left(B_{1}(u, \phi), \psi\right)=\int_{\Omega}[(u \cdot \nabla) \phi] \psi d x
$$

for $u \in C^{\infty}\left(\bar{\Omega}, \mathbb{R}^{\mathrm{N}}\right), \phi, \psi \in C^{\infty}(\bar{\Omega})$. Finally, we define

$$
b_{\Gamma}(u, \phi, \psi):=\left(B_{\Gamma}(u, \phi), \psi\right)_{\Gamma}=\int_{\Gamma}\left(u_{\tau} \cdot \nabla_{\tau} \phi\right) \psi d S,
$$

for $u \in C^{\infty}\left(\bar{\Omega}, \mathbb{R}^{\mathrm{N}}\right) \cap \mathbb{C}_{\text {div }}^{\infty}(\bar{\Omega})$ and $\phi, \psi \in C^{\infty}(\Gamma)$. The operators $B_{0}, B_{1}$ and $B_{\Gamma}$ enjoy continuity properties which generally depend on the space dimension. We summarize some of their important properties in Section 4.

We are now in a position to formulate our problem (1.1)-(1.10) in a weak form. In order to do so, it is more convenient to introduce the unknown function $\psi:=\operatorname{tr}_{\mathrm{D}}(\phi)$ and to interpret (1.8) as an additional second-order parabolic equation on $\Gamma \times(0, \infty)$. To this purpose, we define the Hilbert space $\mathbb{Y}_{\gamma}:=\mathbb{H} \times V_{\gamma}^{1}$, endowed with the scalar product whose associated norm is given by

$$
\|(u, \phi, \psi)\|_{\mathbb{Y}_{\gamma}}^{2}:=|u|^{2}+\|\nabla \phi\|_{2}^{2}+\zeta\|\psi\|_{2, \Gamma}^{2}+\gamma\left\|\nabla_{\tau} \psi\right\|_{2, \Gamma}^{2} \cdot
$$

The notion of a (global) weak solution to our problem is given by

Definition 2.1. Let $\left(u_{0}, \phi_{0}, \psi_{0}\right) \in \mathbb{Y}_{\gamma}$ and $h \in L_{\text {loc }}^{2}\left(0, \infty ;\left(\mathbb{H}^{1}\right)^{*}\right)$. A triplet $(u, \phi, \psi)$ such that $\psi:=\operatorname{tr}_{\mathrm{D}}(\phi)$ almost everywhere in $(0, \infty)$ and

$$
\left\{\begin{array}{l}
(u, \phi, \psi) \in L_{l o c}^{\infty}\left(0, \infty ; \mathbb{Y}_{\gamma}\right), \\
u \in L_{l o c}^{2}\left(0, \infty ; \mathbb{H}^{1}\right) \\
\mu \in L_{l o c}^{2}\left(0, \infty ; H^{1}(\Omega)\right), \\
\mathcal{L}(\psi) \in L_{l o c}^{2}\left(0, \infty ; L^{2}(\Gamma)\right),
\end{array}\right.
$$

with

$$
(\phi, \psi) \in L_{l o c}^{2}\left(0, \infty ; V_{\gamma}^{2}\right)
$$




$$
\partial_{t} \phi \in L_{l o c}^{2}\left(0, \infty ;\left(H^{1}\right)^{*}\right), \partial_{t} \psi \in L_{l o c}^{q}\left(0, \infty ; L^{2}(\Gamma)\right), \partial_{t} u \in L_{l o c}^{p}\left(0, \infty ;\left(\mathbb{H}^{1}\right)^{*}\right),
$$

where

$$
q=4 / 3 \text { if } N=3, \quad q=2-\iota \text { if } N=2,
$$

and $p=4 /(3+2 \delta)$ if $N=3$ and $p=q$ if $N=2$, for some $\delta, \iota>0$, is called weak solution to (1.1)-(1.10) on $(0, T)$, for every $T>0$, if

$$
\begin{aligned}
& \left\langle\partial_{t} u(t), v\right\rangle+a_{0}(u(t), v)+b_{0}(u(t), u(t), v) \\
& =b_{1}(v, \phi(t), \mu(t))+\left(\mathcal{L}(\psi(t)) \nabla_{\tau} \psi(t), v_{\tau}\right)_{\Gamma}+(h(t), v),
\end{aligned}
$$

for all $v \in \mathbb{H}^{1}$ and for almost any $t \in(0, T)$, and

$$
\begin{gathered}
\left\langle\partial_{t} \phi(t), \varphi\right\rangle+(\nabla \mu(t), \nabla \varphi)+b_{1}(u(t), \phi(t), \varphi)=0, \\
\left(\partial_{t} \psi(t), \eta\right)_{\Gamma}+b_{\Gamma}(u(t), \psi(t), \eta)+(\mathcal{L}(\psi(t)), \eta)_{\Gamma}=0,
\end{gathered}
$$

for all $\varphi \in H^{1}(\Omega), \eta \in L^{2}(\Gamma)$ and almost any $t \in(0, T)$, with

$$
\left\{\begin{array}{l}
\mu=-\Delta \phi+f(\phi), \text { a.e. in } \Omega \times(0, T), \\
\mathcal{L}(\psi)=\gamma A_{\tau} \psi+\partial_{n} \phi+\zeta \psi+g(\psi), \text { a.e. on } \Gamma \times(0, T),
\end{array}\right.
$$

and the initial conditions

$$
u_{\mid t=0}=u_{0}, \quad \phi_{\mid t=0}=\phi_{0}, \quad \psi_{\mid t=0}=\psi_{0} .
$$

Remark 2.1. On account of $(2.3)-(2.5)$, we have $u \in C\left([0, T] ;\left(\mathbb{H}^{1}\right)^{*}\right)$ and $(\phi, \psi) \in C\left([0, T] ; V_{\gamma}^{0}\right)$, so that initial conditions (2.10) make sense.

\section{Main Results}

We state our assumptions on $f, g \in C^{1}(\mathbb{R})$ first. Let $m, l \in[1, \infty)$ be fixed, but otherwise arbitrary if $N=2$ and $m=2$ if $N=3$, with $l \in[1, \infty)$ arbitrary if $N=3$.

(a) There exist $c_{G} \geq 0, C_{g}>0$ such that

$$
\left|g^{\prime}(s)\right| \leq C_{g}\left(1+|s|^{l}\right), \quad G^{\prime \prime}(s) \geq-c_{G}, \quad G(s) \geq-c_{G}
$$

for any $s \in \mathbb{R}$.

(b) There exist $c_{F} \geq 0, C_{f}>0$ such that

$$
\left|f^{\prime}(s)\right| \leq C_{f}\left(1+|s|^{m}\right), \quad F^{\prime \prime}(s) \geq-c_{F}, \quad F(s) \geq-c_{F}
$$

for any $s \in \mathbb{R}$. Here, $F(r)=\int_{0}^{r} f(\zeta) d \zeta$ and $G(r)=\int_{0}^{r} g(\zeta) d \zeta$.

Let us make some preliminary (formal) considerations on the total energy associated with our initial and boundary value problem. This consists of kinetic and potential energies and it is given by

$$
\mathcal{E}(u(t), \phi(t), \psi(t)):=\frac{1}{2}\|(u(t), \phi(t), \psi(t))\|_{\mathbb{Y}_{\gamma}}^{2}+\int_{\Omega} F(\phi(t)) d x+\int_{\Gamma} G(\psi(t)) d S .
$$

In order to show that $\mathcal{E}$ is decreasing, we need to perform some basic computations which require a sufficiently smooth solution $(u, \phi, \psi)$. Recalling that $b_{0}(u, u, u)=0$, for $u \in \mathbb{H}^{1}$, we can take $v=u(t)$ in $(2.6)$ to deduce that

$$
\begin{aligned}
& \frac{1}{2} \frac{d}{d t}|u(t)|^{2}+a_{0}(u(t), u(t))-b_{1}(u(t), \phi(t), \mu(t)) \\
& =b_{\Gamma}\left(u_{\tau}(t), \psi(t), \mathcal{L}(\psi(t))\right)+\langle h(t), u(t)\rangle .
\end{aligned}
$$

Next, pairing the equation of (2.7) with $\varphi=\mu(t)$ and equation $(2.8)$ with $\eta=\mathcal{L}(\psi(t))$, respectively, and then testing each one of $(2.9)$ with $\partial_{t} \phi(t)$ and $\partial_{t} \psi(t)$ in $L^{2}(\Omega)$ and $L^{2}(\Gamma)$, respectively, we deduce that

$$
\begin{aligned}
& \frac{1}{2} \frac{d}{d t}\left(\|\nabla \phi(t)\|_{2}^{2}+\gamma\left\|\nabla_{\tau} \psi(t)\right\|_{2, \Gamma}^{2}+\zeta\|\psi(t)\|_{2, \Gamma}^{2}+2(G(\psi(t)), 1)_{\Gamma}+2(F(\phi(t)), 1)\right) \\
& =-\|\nabla \mu(t)\|_{2}^{2}-b_{1}(u(t), \phi(t), \mu(t))-b_{\Gamma}\left(u_{\tau}(t), \psi(t), \mathcal{L}(\psi(t))\right)-\|\mathcal{L}(\psi(t))\|_{2, \Gamma}^{2},
\end{aligned}
$$

for $t \in(0, T)$ and for any given $T>0$. Next, for every $\sigma>0$, we have

$$
\langle h(t), u(t)\rangle \leq(4 \sigma)^{-1}\|h(t)\|_{\left(H^{1}(\Omega)\right)^{*}}^{2}+\sigma\|u(t)\|^{2} .
$$


Thus, we can absorb this term on the right-hand side of (3.4) by choosing a suitable $\sigma \ll$ $\min \{\nu, \beta\}$. Adding together equalities (3.4)-(3.5) and integrating the resulting relation over $(0, t)$, we find that $\mathcal{E}$ satisfies an energy inequality provided that $(u, \phi, \psi)$ is regular enough. More precisely, there holds

$$
\begin{aligned}
& \mathcal{E}(u(t), \phi(t), \psi(t))+\int_{0}^{t}\left(C_{\sigma}\|u(s)\|^{2}+\|\nabla \mu(s)\|_{2}^{2}+\|\mathcal{L}(\psi(s))\|_{2, \Gamma}^{2}\right) d s \\
& \leq \mathcal{E}\left(u_{0}, \phi_{0}, \psi_{0}\right)+(4 \sigma)^{-1} \int_{0}^{t}\|h(s)\|_{\left(H^{1}(\Omega)\right)^{*}}^{2} d s,
\end{aligned}
$$

for almost any $t \in(0, T)$ and for some $C_{\sigma}>0$. It follows from (3.6) that $(u, \phi, \psi)$ belongs to the functional class $(2.3)$. We note that $\mathcal{E}\left(u_{0}, \phi_{0}, \psi_{0}\right)<\infty$ is equivalent to having $\left(u_{0}, \phi_{0}, \psi_{0}\right) \in \mathbb{Y}_{\gamma}$, owing to (3.1) and (3.2). Note that, if $h \equiv 0$, then we have

$$
\begin{aligned}
& \mathcal{E}(u(t), \phi(t), \psi(t))+\int_{0}^{t}\left(a_{0}(u(y), u(y))+\|\nabla \mu(y)\|_{2}^{2}+\|\mathcal{L}(\psi(y))\|_{2, \Gamma}^{2}\right) d y \\
& \leq \mathcal{E}\left(u_{0}, \phi_{0}, \psi_{0}\right) .
\end{aligned}
$$

Remark 3.1. We note that in order to derive (3.5) (and, therefore, (3.6)), the choices $\varphi=\mu(t)$ and $\eta=\mathcal{L}(\psi(t))$ in (2.7)-(2.8) are indeed crucial. Unfortunately, at the level of Galerkin truncations, in order to perform basic energy estimates, such choices are not allowed. Indeed, such a procedure would necessarily require that $\operatorname{tr}_{\mathrm{D}}(\varphi)=\eta$ at the level of truncations. This is the case of the sole Cahn-Hilliard equation with dynamic boundary conditions for which the validity of (3.5) can be always achieved by choosing $\varphi=\partial_{t} \phi(t)$ and $\eta=\partial_{t} \psi(t)$. However, such choices can no longer be exploited because of $B_{\Gamma}\left(u_{\tau}, \psi\right)$ (see $\left.(2.8)\right)$.

These considerations motivate us to introduce a further notion of a weak solution which will play a crucial role in the study of the longtime behavior as well as the existence of non-regular solutions.

Definition 3.1. A triplet $(u, \phi, \psi)$ is an energy solution (or weak solution with finite energy) if it is a solution in the sense of Definition 2.1 and satisfies the energy inequality (3.6).

Our first main result is the following

Theorem 3.2. Let $f, g \in C^{1}(\mathbb{R})$ satisfy assumptions $(a)$-(b). If $\left(u_{0}, \phi_{0}, \psi_{0}\right) \in \mathbb{Y}_{\gamma}$ and $h \in$ $L_{l o c}^{2}\left(0, \infty ;\left(\mathbb{H}^{1}\right)^{*}\right)$ then there exists at least one global energy solution $(u, \phi, \psi) \in L_{l o c}^{\infty}\left(0, \infty ; \mathbb{Y}_{\gamma}\right)$. If $h \equiv 0$, then there exists an energy solution which satisfies the following (strong) energy inequality

$$
\begin{aligned}
& \mathcal{E}(u(t), \phi(t), \psi(t))+\int_{s}^{t}\left(a_{0}(u(y), u(y))+\|\nabla \mu(y)\|_{2}^{2}+\|\mathcal{L}(\psi(y))\|_{2, \Gamma}^{2}\right) d y \\
& \leq \mathcal{E}(u(s), \phi(s), \psi(s))
\end{aligned}
$$

for all $t \geq s$ and for almost any $s \in[0, \infty)$, including $s=0$.

The second main result is concerned with the asymptotic behavior of energy solutions as time tends to infinity. The proof is based on a suitable version of the Eojasiewicz-Simon inequality and the results obtained in the next sections. We emphasize that our subsequent results hold for any energy weak solution which satisfies a stronger energy inequality, even though uniqueness is not known to hold for these solutions as well. In particular, the asymptotic stabilization (as time goes to infinity) of the global energy solution holds not only for the limit points obtained from the regularization scheme exploited in Section 5, but also for the ones obtained from other numerical schemes in which the strong energy inequality (3.8) can be proven. In other words, the subsequent result provides a further selection criterion in order to eliminate all non-physical weak solutions which may nonetheless satisfy Definition 2.1 without fulfilling an energy inequality. For the sake of simplicity, in the next statement we will assume $h \equiv 0$ and we denote by $H^{1-}(\Omega)$ and $H^{1-}(\Gamma)$ the spaces $H^{1-\eta}(\Omega)$ and $H^{1-\eta}(\Gamma)$, respectively, for some $\eta \in(0,1)$.

Theorem 3.3. Let the assumptions of Theorem 3.2 be satisfied and assume that $F, G$ are real analytic. Let $(u, \phi, \psi)$ be an energy solution corresponding to some $\left(u_{0}, \phi_{0}, \psi_{0}\right) \in \mathbb{Y}_{\gamma}$ which satisfies (3.8). Then, as $t$ goes to infinity, we have

$$
\phi(t) \rightarrow \phi_{*} \text { strongly in } H^{1-}(\Omega), \psi(t) \rightarrow \psi_{*} \text { strongly in } H^{1-}(\Gamma)
$$


and

$$
u(t) \rightarrow 0 \text { weakly in } \mathbb{H} \text {, strongly in }\left(\mathbb{H}^{1}\right)^{*},
$$

where $\left(\phi_{*}, \psi_{*}\right) \in V_{\gamma}^{2} \cap\left\{\phi_{*}:\left\langle\phi_{*}\right\rangle=\left\langle\phi_{0}\right\rangle\right\}$ is a stationary solution, namely,

$$
-\Delta \phi_{*}+f\left(\phi_{*}\right)=\text { const., in } \Omega,-\gamma A_{\tau} \psi_{*}+\partial_{n} \phi_{*}+\zeta \psi_{*}+g\left(\psi_{*}\right)=0 \text {, on } \Gamma \text {. }
$$

Remark 3.2. Concerning (3.9), the following convergence rate

$$
\left\|\phi(t)-\phi_{*}\right\|_{H^{1-}}+\left\|\psi(t)-\psi_{*}\right\|_{H^{1-}(\Gamma)} \lesssim(1+t)^{-\chi}
$$

holds for some $\chi \in(0,1)$, depending on $\left(\phi_{*}, \psi_{*}\right)$ (see also Remark 6.2).

If the energy solution of Theorem 3.3 becomes more regular on the boundary, then the following conditional result holds.

Theorem 3.4. Let the assumptions of Theorem 3.3 be satisfied and $h \equiv 0$. Further, assume that a global weak solution $(u, \phi, \psi)$ to problem (1.1)-(1.10) can be deduced as the limit point of some approximating solutions $\left(u_{\epsilon}, \phi_{\epsilon}, \psi_{\epsilon}\right)$ that have the following properties:

(i) for some $s>\frac{N}{2}-1$,

$$
\psi_{\epsilon} \in L^{\infty}\left(0, \infty ; H^{1+s}(\Gamma)\right) \text { uniformly in } \epsilon>0 .
$$

(ii) $\left(u_{\epsilon}, \phi_{\epsilon}, \psi_{\epsilon}\right)_{\mid t=0} \rightarrow\left(u_{0}, \phi_{0}, \psi_{0}\right)$ in $\mathbb{Y}_{\gamma}$, and the energy identity (3.4) and the inequality (3.8) holds for $\left(u_{\epsilon}, \phi_{\epsilon}, \psi_{\epsilon}\right)$.

Then the weak solution $(u, \phi, \psi)$ is such that

$$
u(t) \rightarrow 0 \text { strongly in } \mathbb{H} \text { as } t \rightarrow \infty .
$$

Remark 3.3. The regularity stated in (3.13) is optimal in the sense that it cannot be improved. In three dimensions, we note that (3.13) gives a sufficient condition on the order parameter rather than the velocity and emphasizes again the strong coupling of the system through the boundary.

Our final result concerns the case of a logarithmic-like potential (cf. (1.5)). More precisely, we suppose that $F \in C^{2}(-1,1) \cap C[-1,1]$ and $f=F^{\prime} \in C^{1}(-1,1)$ can be decomposed into $f=f_{0}+f_{1}$, with $f_{0}$ satisfying

$$
\left\{\begin{array}{l}
\lim _{s \rightarrow \pm 1} f_{0}(s)= \pm \infty, \quad \lim _{s \rightarrow \pm 1} f_{0}^{\prime}(s)=\infty, \\
f_{0}(0)=0, f_{0}^{\prime}(s) \geq 0, \text { for any } s \in(-1,1),
\end{array}\right.
$$

while $f_{1} \in C^{1}(\mathbb{R})$ is a regular function such that $f_{1}^{\prime} \in L^{\infty}(\mathbb{R})$.

Then we can prove the existence of a global weak solution. Indeed we have

Theorem 3.5. Let $f=f_{0}+f_{1} \in C^{1}(-1,1), \widetilde{g}(s):=g(s)+\zeta s \in C^{1}(\mathbb{R})$ satisfy assumptions (3.15), (3.1) and the following conditions: there exist constants $M \in(0,1), \delta>0, C_{M}>0$, $C_{\delta, M}>0$ such that

$$
f_{0}(s) g(s) \geq-C_{M}, \quad \text { for any } s \in(-1,-M] \cup[M, 1)
$$

and

$$
f_{0}^{\prime}(s)-\delta\left(f_{0}(s)\right)^{2} \geq-C_{\delta, M}, \quad \text { for any } s \in(-1,-M] \cup[M, 1) .
$$

If $\left(u_{0}, \phi_{0}, \psi_{0}\right) \in \mathbb{H} \times V_{\gamma}^{1}, F\left(\phi_{0}\right) \in L^{1}(\Omega) \oplus L^{1}(\Gamma),\left\langle\phi_{0}\right\rangle \in(-1,1)$, and $h \in L_{\text {loc }}^{2}\left(0, \infty ;\left(\mathbb{H}^{1}\right)^{*}\right)$, then there exists at least one global energy solution in the sense of Definition 2.1 satisfying (2.3)-(2.5) and, in addition,

$$
F(\phi) \in L_{l o c}^{\infty}\left(0, \infty ; L^{1}(\Omega) \oplus L^{1}(\Gamma)\right), \quad f_{0}(\phi) \in L_{l o c}^{2}\left(0, \infty ; L^{2}(\Omega)\right) .
$$

Thus $\phi(x, t) \in(-1,1)$ for almost any $(x, t) \in \Omega \times(0, \infty)$ and $\phi(x, t) \in[-1,1]$ for almost any $(x, t) \in \Gamma \times(0, \infty)$. If $h \equiv 0$, the (strong) energy inequality (3.8) holds for all $t \geq s$ and for almost any $s \in[0, \infty)$, including $s=0$.

Remark 3.4. Condition (3.17) may seem a bit cumbersome, but in fact it can be easily checked for a wide range of nonlinearities satisfying (3.15). For instance, condition (3.17) is fulfilled by the logarithmic density function

$$
f_{0}(s)=c_{0} \ln \left(\frac{1+s}{1-s}\right), c_{0}>0 .
$$


We also emphasize the role of the surface diffusion mechanism for (3.17). In particular, such an assumption requires that $\gamma>0$ (see the proof of Theorem 3.5, (7.14)). Finally, when (3.15) is in full force then condition (3.16) is synonymous with the condition that $\pm g( \pm 1)>0$, i.e., $g$ shares the same sign as the singular potential $f$ near the singular points \pm 1 . This kind of sign conditions is natural and has appeared elsewhere (see [20] and references therein).

\section{TECHNICAL TOOLS}

In this section we report some additional technical tools which are necessary for our analysis. We first recall some properties of spatial mollifiers over compact manifolds and prove some additional inequalities of Poincaré-Young type.

Lemma 4.1. (cf. [70]) Let $M$ be a (sufficiently smooth) compact manifold with or without boundary. There exists a mollifier $\mathcal{J}_{\epsilon}, 0<\epsilon \leq 1$, such that, for each $\varphi \in H^{m}(M), \mathcal{J}_{\epsilon} \varphi \in C^{\infty}(M)$. Moreover, the following properties also hold:

(i) $\left(\mathcal{J}_{\epsilon} \varphi, \psi\right)_{L^{2}(M)}=\left(\varphi, \mathcal{J}_{\epsilon} \psi\right)_{L^{2}(M)}$, i.e., $\mathcal{J}_{\epsilon}$ is self-adjoint.

(ii) $\mathcal{J}_{\epsilon}$ commutes with distributional derivatives, that is,

$$
D^{\lambda} \mathcal{J}_{\epsilon} \varphi=\mathcal{J}_{\varepsilon} D^{\lambda} \varphi, \text { for any }|\lambda| \leq m, \varphi \in H^{m}(M) .
$$

(iii) For any $\varphi \in H^{m}(M), \mathcal{J}_{\varepsilon} \varphi \rightarrow \varphi$ in $H^{m}(M)$ as $\varepsilon$ goes to 0 .

(iv) There exists a constant $C_{m k}>0$ such that

$$
\left\|\mathcal{J}_{\epsilon} \varphi\right\|_{H^{m+k}} \leq \frac{C_{m k}}{\varepsilon^{k}}\|\varphi\|_{H^{m}}, \quad \text { forall } \varphi \in H^{m}(M)
$$

for $m, k \in \mathbb{N} \cup\{0\}$.

Remark 4.1. On compact manifolds without boundary, such as $M=\Gamma$, one can use the standard Friedrichs mollifier for $\mathcal{J}_{\epsilon}$ in order to apply Lemma 4.1 (see [70]). In the case of bounded domains $\Omega \subset \mathbb{R}^{N}$ with boundary $\Gamma$, we let $M=\Omega$ be an open subset (with closure $\bar{M}$ ) of the compact Riemannian manifold $\widetilde{M}$ without boundary, of dimension $N+1$, and let $E: H^{m}(M) \rightarrow H^{m}(\widetilde{M})$ be an extension operator, as constructed in [69, Chapter 4, pp. 333]. If $R: H^{m}(\widetilde{M}) \rightarrow H^{m}(M)$ is the restriction operator, then the corresponding mollifier $\mathcal{J}_{\varepsilon}$ can be defined as $\mathcal{J}_{\epsilon} \varphi=R \widetilde{\mathcal{J}}_{\varepsilon} E \varphi$, where $\widetilde{\mathcal{J}}_{\varepsilon}$ is a Friedrichs mollifier on $\widetilde{M}$.

We now prove the following inequality of Poincaré-Young type.

Lemma 4.2. For all $\varepsilon \in(0,1)$, there is $\alpha>0$ such that

$$
\|\varphi\|_{2, \Gamma}^{2} \leq \varepsilon\|\nabla \varphi\|_{2}^{2}+\varepsilon^{-\alpha}\|\varphi\|_{2}^{2}, \quad \text { for all } \varphi \in H^{1}(\Omega) \text {. }
$$

Proof. Clearly, $\|\nabla \varphi\|_{2}+\|\varphi\|_{2}$ is an equivalent norm on $H^{1}(\Omega)$. By a scaling argument, it suffices to prove the inequality for $\|\varphi\|_{2, \Gamma}=1$. Suppose that there is no $\alpha>0$ such that the inequality holds for a given $\varepsilon \in(0,1)$. Then, for any $k \in \mathbb{N}$, there is $\varphi_{k} \in H^{1}(\Omega)$ such that

$$
\left\|\varphi_{k}\right\|_{2, \Gamma}^{2}=1 \geq \varepsilon\left\|\nabla \varphi_{k}\right\|_{2}^{2}+\varepsilon^{-k}\left\|\varphi_{k}\right\|_{2}^{2} .
$$

It follows from this inequality that the resulting sequence $\left\{\varphi_{k}\right\}$ is bounded in $H^{1}(\Omega)$. Since the trace operator is a compact map from $H^{1}(\Omega)$ into $L^{2}(\Gamma)$ and the identity operator is also compact from $H^{1}(\Omega)$ into $L^{2}(\Omega)$, we find a subsequence, again denoted by $\left\{\varphi_{k}\right\}$, that converges strongly in $L^{2}(\Gamma)$ and in $L^{2}(\Omega)$ to some $\varphi$. By assumption, we have $\|\varphi\|_{2, \Gamma}=1$. On the other hand, the inequality shows that $\left\|\varphi_{k}\right\|_{2}^{2} \leq \varepsilon^{k}$ for all $k$, so that $\|\varphi\|_{2}=0$ and thus $\varphi=0$ almost everywhere in $\Omega$, which yields $\varphi_{\mid \Gamma}=0$ almost everywhere, due to boundedness of the trace map, hence a contradiction.

With the help of Lemma 4.2, the proof can easily be adapted to the following case.

Lemma 4.3. For all $\varepsilon \in(0,1)$ there is $\alpha>0$ such that

$$
\left\|u_{\tau}\right\|_{2, \Gamma}^{2} \leq \varepsilon|D(u)|^{2}+\varepsilon^{-\alpha}|u|^{2},
$$

for all $u \in \mathbb{H}^{1}$, with $\mathbb{H}^{1}$ defined by (2.1). Here $|\cdot|$ denotes the $\mathbb{L}^{2}(\Omega)$-norm.

We also recall the compactness lemma of Aubin-Lions-Simon type (see, for instance, [48] in the case $q>1$ and [64] when $q=1$ ) and a weak convergence criterion in $L^{p}$-spaces (see, for instance, $[19])$. 
Lemma 4.4. Let $X_{0} \Subset X_{1} \subset X_{2}$ be an inclusion of Banach spaces with the first one being compact. Let $1<p \leq \infty, 1 \leq q \leq \infty$ and $I$ be a bounded subinterval of $\mathbb{R}$. Then, the sets

$$
\left\{\varphi \in L^{p}\left(I ; X_{0}\right): \partial_{t} \varphi \in L^{q}\left(I ; X_{2}\right)\right\} \Subset L^{p}\left(I ; X_{1}\right) \text {, if } 1<p<\infty,
$$

and

$$
\left\{\varphi \in L^{p}\left(I ; X_{0}\right): \partial_{t} \varphi \in L^{q}\left(I ; X_{2}\right)\right\} \Subset C\left(I ; X_{1}\right) \text {, if } p=\infty, q>1,
$$

with compact inclusions.

Lemma 4.5. Let $O$ be a bounded domain in $\mathbb{R} \times \mathbb{R}^{N}$ and let a sequence $q_{n} \in L^{p}(O), p \in(1, \infty)$, be given. Assume that $\left\|q_{n}\right\|_{L^{p}(O)} \leq C$, with $C>0$ independent of $n, q_{n} \rightarrow q$ almost everywhere on $O$ and $q \in L^{p}(O)$. Then as $n \rightarrow \infty, q_{n} \rightarrow q$ weakly in $L^{p}(O)$.

Moreover, we need the following basic result on elliptic regularity for an elliptic boundary value problem for $(\phi, \psi)$ with $\psi=\operatorname{tr}_{\mathrm{D}}(\phi)$ (see, for instance, [53, Lemma A.1]).

Lemma 4.6. For each $\gamma>0$, consider the following linear boundary value problem

$$
\begin{aligned}
-\Delta \phi & =h_{1}, \quad \text { in } \Omega, \\
-\gamma \Delta_{\tau} \psi+\partial_{n} \phi+\zeta \psi & =h_{2}, \quad \text { on } \Gamma,
\end{aligned}
$$

where $\left(h_{1}, h_{2}\right) \in L^{2}(\Omega) \times L^{2}(\Gamma)$. Then the following estimate holds

$$
\|\phi\|_{H^{2}}+\|\psi\|_{H^{2}(\Gamma)} \leq C\left(\left\|h_{1}\right\|_{2}+\left\|h_{2}\right\|_{2, \Gamma}\right),
$$

for some constant $C>0$ independent of $(\phi, \psi)$.

As far as the trilinear forms are concerned, we have the following basic properties.

Lemma 4.7. Let $1 \leq s_{1}, s_{2}, s_{3} \leq \infty$ be such that $s_{1}^{-1}+s_{2}^{-1}+s_{3}^{-1}=1$ and $1 \leq p_{1}, p_{2}, p_{3} \leq \infty$ be such that $p_{1}^{-1}+p_{2}^{-1}+p_{3}^{-1}=1$.

(i) The forms $b_{j}, j=0,1$ and $b_{\Gamma}$ extend uniquely to continuous trilinear forms as follows:

$$
\begin{aligned}
& b_{j}: \mathbb{L}_{\text {div }}^{p_{1}}(\Omega) \times \mathbb{W}^{1, p_{2}}(\Omega) \times \mathbb{L}^{p_{3}}(\Omega) \rightarrow \mathbb{R}, \\
& b_{\Gamma}:\left(\mathbb{L}^{p_{1}}(\Gamma) \cap \mathbb{H}\right) \times W^{1, p_{2}}(\Gamma)^{N-1} \times L^{p_{3}}(\Gamma) \rightarrow \mathbb{R} .
\end{aligned}
$$

(ii) Let $T>0$ be fixed and define for $u, v, w \in \mathbb{C}^{\infty}([0, T] \times \bar{\Omega}), \phi, \psi \in C^{\infty}([0, T] \times \bar{\Omega})$ the functionals

$$
\begin{aligned}
b_{0}^{T}(u, v, w) & :=\int_{0}^{T} b_{0}(u(t), v(t), w(t)) d t, \\
b_{1}^{T}(u, \phi, \psi) & :=\int_{0}^{T} b_{0}(u(t), \phi(t), \psi(t)) d t, \\
b_{\Gamma}^{T}(u, \phi, \psi) & :=\int_{0}^{T} b_{\Gamma}(u(t), \phi(t), \psi(t)) d t .
\end{aligned}
$$

Then, $b_{\Gamma}^{T}$ and $b_{j}^{T}, j=0,1$, extend uniquely to trilinear continuous forms as follows:

$$
\begin{aligned}
& b_{j}^{T}:\left(L^{s_{1}} 0, T ; \mathbb{L}_{d i v}^{p_{1}}(\Omega)\right) \times L^{s_{2}}\left(0, T ; \mathbb{W}^{1, p_{2}}(\Omega)\right) \times L^{s_{3}}\left(0, T ; \mathbb{L}^{p_{3}}(\Omega)\right) \rightarrow \mathbb{R}, \\
& b_{\Gamma}: L^{s_{1}}\left(0, T ; \mathbb{L}^{p_{1}}(\Gamma) \cap \mathbb{H}\right) \times L^{s_{2}}\left(0, T ; W^{1, p_{2}}(\Gamma)^{N-1}\right) \times L^{s_{3}}\left(0, T ; L^{p_{3}}(\Gamma)\right) \rightarrow \mathbb{R} .
\end{aligned}
$$

Proof. The proof is achieved through repeated application of proper Hölder's inequalities.

Finally, we report a fundamental result on pointwise multiplication of functions in Sobolev spaces on $\Gamma$ (see [51]).

Lemma 4.8. Let $s, s_{1}, s_{2} \in \mathbb{R}$ be such that

$$
s_{1}+s_{2} \geq 0, \quad \min \left(s_{1}, s_{2}\right) \geq s \quad \text { and } \quad s_{1}+s_{2}-s>\frac{N-1}{2},
$$

where the strictness of the last two inequalities can be interchanged if $s \in \mathbb{N}_{0}$. Then, the pointwise multiplication of functions extends uniquely to a continuous bilinear map from $H^{s_{1}}(\Gamma) \otimes H^{s_{2}}(\Gamma)$ to $H^{s}(\Gamma)$. 
We conclude this section with a number of elementary results for some general linear problems associated with the nonstationary Stokes equation subject to a Navier boundary condition and the (viscous) Cahn-Hilliard equation with a dynamic boundary condition. To this end, we first consider the Stokes problem

$$
\begin{aligned}
& \partial_{t} u-\operatorname{div}(2 \nu D(u))+\nabla p=h_{1}, \\
& \operatorname{div}(u)=0,
\end{aligned}
$$

in $\Omega \times(0, \infty)$, subject to boundary conditions of the form

$$
\begin{aligned}
& u \cdot n=0, \\
& 2 \nu(D(u) \cdot n)_{\tau}+\beta u_{\tau}=h_{2},
\end{aligned}
$$

on $\Gamma \times(0, \infty)$, and an initial condition

$$
u_{\mid t=0}=u_{0} .
$$

We have the following result on the solvability of problem (4.1)-(4.5). We recall that $\Omega \subset \mathbb{R}^{N}$ is bounded and $N \leq 3$.

Theorem 4.9. Let $h_{1} \in L^{2}\left(0, T ;\left(\mathbb{H}^{1}\right)^{*}\right)$ and $h_{2} \in L^{2}\left(0, T ; \mathbb{L}^{4 / 3}(\Gamma)\right)$ for some $T>0$. For every $u_{0} \in \mathbb{H}$, system (4.1)-(4.5) possesses a unique weak solution $u$ on the interval $[0, T]$ such that

$$
u \in L^{\infty}(0, T ; \mathbb{H}) \cap L^{2}\left(0, T ; \mathbb{H}^{1}\right) \cap H^{1}\left(0, T ;\left(\mathbb{H}^{1}\right)^{*}\right) .
$$

Moreover there exists a constant $C>0$, independent of time, $u$ and the initial datum, such that

$$
\begin{aligned}
& \|u\|_{L^{\infty}(0, T ; \mathbb{H})}^{2}+\|u\|_{L^{2}\left(0, T ; \mathbb{H}^{1}\right) \cap H^{1}\left(0, T ;\left(\mathbb{H}^{1}\right)^{*}\right)}^{2} \\
\leq & \left|u_{0}\right|^{2}+C\left\|h_{2}\right\|_{L^{2}\left(0, T ; \mathbb{L}^{4 / 3}(\Gamma)\right)}^{2}+C\left\|h_{1}\right\|_{L^{2}\left(0, T ;\left(\mathbb{H}^{1}\right)^{*}\right)}^{2} .
\end{aligned}
$$

Proof. For the sake of completeness, we give a proof of this basic result for (4.1)-(4.5). A similar problem was also investigated in [2] in the case $h_{2}=0$. In order to show the existence of a weak solution, we make use of a Galerkin scheme to project on a finite dimensional space as in [71]. The corresponding bound (4.7) for the truncated solution is also used to extract a subsequence which converges weakly to a certain vector-valued function, candidate to be the solution to the original problem. We take the family $\left\{v_{j}\right\}, j \in \mathbb{N}_{+}$, in $\mathbb{H}$, of orthonormal eigenfunctions of the Stokes operator $A_{0}$ as a Galerkin basis in $\mathbb{H}^{1}=D\left(A_{0}^{1 / 2}\right)$. For $n \in \mathbb{N}$, we consider the projection

$$
\mathcal{O}_{n}: D\left(A_{0}^{1 / 2}\right) \rightarrow \mathcal{M}^{n}=\operatorname{span}\left\{v_{1}, v_{2}, \ldots, v_{n}\right\}
$$

and set

$$
\mathcal{M}^{\infty}=\cup_{n=1}^{\infty} \mathcal{M}^{n}
$$

Clearly, $\mathcal{M}^{\infty}$ is a dense subspace of $\mathbb{H}^{1}$. Thus, for each given $n \in \mathbb{N}$, the approximating problem associated with (4.1)-(4.5) is given by

Problem $\mathbf{P}_{n}$. Find $t_{n} \in(0, T]$ and $\mathbf{a}:=\mathbf{a}^{n} \in C^{1}\left(\left[\tau, t_{n}\right] ; \mathbb{R}^{n}\right)$ such that

$$
u_{n}(t)=\sum_{j=1}^{n} a_{j}(t) v_{j}
$$

solves the system

$$
\left(\partial_{t} u_{n}(t), v\right)+a_{0}\left(u_{n}(t), v\right)=\left(h_{1}(t), v\right)+\left(h_{2}(t), v\right)_{\Gamma},
$$

for every $t \in\left(0, t_{n}\right)$ and every $v \in \mathcal{M}^{n}$, with

$$
u_{n}(0)=\mathcal{O}_{n} u_{0}
$$

Clearly, one can write (4.9)-(4.10) as a Cauchy problem for a linear system of ordinary differential equations, by making the choice $v=v_{i}$ in $(4.9)$, for $i=1, \ldots, n$. Equation (4.9) can be used to deduce a uniform bound on the solutions $u_{n}$. Such an estimate, in particular, ensures that we can take $t_{n}=T$, for every $n$. Indeed, taking $v=u_{n}$ in (4.9), we infer

$$
\begin{aligned}
\frac{d}{d t}\left|u_{n}(t)\right|^{2}+2\left\|u_{n}(t)\right\|^{2} & =2\left(h_{1}(t), u_{n}(t)\right)+2\left(h_{2}(t), u_{n}(t)\right)_{\Gamma} \\
& \leq \delta\left\|h_{1}(t)\right\|_{\left(\mathbb{H}^{1}\right)^{*}}^{2}+\frac{C}{\delta}\left\|u_{n}(t)\right\|^{2}+\delta\left\|h_{2}(t)\right\|_{4 / 3, \Gamma}^{2},
\end{aligned}
$$


for every $t \in\left[0, t_{n}\right]$, on account of the embedding $H^{1 / 2}(\Gamma) \hookrightarrow L^{4}(\Gamma)$, where $C>0$ is a constant depending at most on the physical parameters of the problem, but independent of $n$. In particular, integrating the foregoing inequality over $(0, t)$ and choosing a sufficiently small $\delta \ll \min \{\nu, \beta\}$, we see that the local solution $u_{n}$ to $\mathbf{P}_{n}$ can be extended up to time $T$, that is, $t_{n}=T$, for every $n$. Furthermore, we have a uniform bound for $\left\{u_{n}\right\}$ in $L^{\infty}(0, T ; \mathbb{H}) \cap L^{2}\left(0, T ; \mathbb{H}^{1}\right)$. This bound, by comparison in (4.9), also provides a uniform bound of $\left\{\partial_{t} u_{n}\right\}$ in $L^{2}\left(0, T ;\left(\mathbb{H}^{1}\right)^{*}\right)$. Thus we can pass to the limit in a standard way and find a function $u$ which is a weak solution to (4.1)-(4.5) and satisfies (4.7). Uniqueness of the weak solution can also be shown by the same estimate, due to the linearity of the problem.

We conclude this section by reporting a result for the linear fourth-order equation

$$
\begin{aligned}
& \partial_{t} \phi-\Delta \widehat{\mu}=l_{1}, \quad \text { in } \Omega \times(0, \infty) \\
& \partial_{n} \widehat{\mu}=0, \quad \text { on } \Gamma \times(0, \infty),
\end{aligned}
$$

endowed with dynamic boundary condition for $\psi=\operatorname{tr}_{\mathrm{D}}(\phi)$,

$$
\partial_{t} \psi+\widehat{\mathcal{L}}(\psi)=l_{3}, \quad \text { on } \Gamma \times(0, \infty),
$$

where $\widehat{\mu}:=-\Delta \phi+\sigma \partial_{t} \phi+l_{2}$ and $\widehat{\mathcal{L}}(\psi):=-\gamma A_{\tau} \psi+\partial_{n} \phi+\zeta \psi$, with $\sigma \in[0,1]$. This system is also endowed with the initial conditions

$$
\phi_{\mid t=0}=\phi_{0}, \quad \text { in } \Omega, \quad \psi_{\mid t=0}=\psi_{0}, \quad \text { on } \Gamma \text {. }
$$

Theorem 4.10. Let $l_{1} \in L^{2}\left(0, T ;\left(H^{1}(\Omega)\right)^{*}\right)$ with $\left\langle l_{1}\right\rangle \equiv 0, l_{2} \in L^{2}\left(0, T ; L^{2}(\Omega)\right)$ and $l_{3} \in$ $L^{2}\left(0, T ; L^{2}(\Gamma)\right)$, for some $T>0$. For every $\left(\phi_{0}, \psi_{0}\right) \in V_{\gamma}^{1}$ and $\sigma \in[0,1]$, system (4.11)-(4.14) possesses a unique weak solution $(\phi, \psi)$ on the interval $[0, T]$ such that

$$
\begin{aligned}
& \left.(\phi, \psi) \in L^{\infty}\left(0, T ; V_{\gamma}^{1}\right) \cap H^{1}\left(0, T ;\left(H^{1}(\Omega)\right)^{*}\right) \times L^{2}(\Gamma)\right), \\
& (\phi, \psi) \in L^{2}\left(0, T ; V_{\gamma}^{2}\right)
\end{aligned}
$$

and

$$
\partial_{t} \phi \in L^{2}\left(0, T ; L^{2}(\Omega)\right), \quad \text { if } \sigma>0
$$

Moreover, the mass is conserved, that is $\langle\phi(t)\rangle=\left\langle\phi_{0}\right\rangle$, for almost any $t>0$, and there exists a constant $C>0$, independent of time, $\sigma \in[0,1],(\phi, \psi)$ and the initial datum, such that the following estimate holds:

$$
\begin{aligned}
& \|(\phi, \psi)\|_{L^{\infty}\left(0, T ; V_{\gamma}^{1}\right)}^{2}+\|(\phi, \psi)\|_{H^{1}\left(0, T ;\left(H^{1}(\Omega)\right)^{*} \times L^{2}(\Gamma)\right)}^{2} \\
& +\sigma\left\|\partial_{t} \phi\right\|_{L^{2}\left(0, T ; L^{2}(\Omega)\right)}^{2}+\|(\phi, \psi)\|_{L^{2}\left(0, T ; V_{\gamma}^{2}\right)}^{2} \\
& +\|\widehat{\mu}\|_{L^{2}\left(0, T ; H^{1}(\Omega)\right)}^{2}+\|\widehat{\mathcal{L}}(\psi)\|_{L^{2}\left(0, T ; L^{2}(\Gamma)\right)}^{2} \\
& \leq C\left(\left\|\left(\phi_{0}, \psi_{0}\right)\right\|_{V_{\gamma}^{1}}^{2}+\left\|l_{1}\right\|_{L^{2}\left(0, T ;\left(H^{1}(\Omega)\right)^{*}\right)}^{2}+\left\|l_{2}\right\|_{L^{2}\left(0, T ; L^{2}(\Omega)\right)}^{2}+\left\|l_{3}\right\|_{L^{2}\left(0, T ; L^{2}(\Gamma)\right)}^{2}\right) .
\end{aligned}
$$

Proof. The proof is given in [53] for the corresponding homogeneous problem exploiting a Schauder fixed point argument when $\sigma>0$. The existence of a solution in the full case $\sigma=0$ is shown in [34] using a Galerkin-type approach, but it can be easily modified to include the additional term when $\sigma>0$. Indeed, the proofs of [34, Section 4, Theorems 4.2 and 4.3] provide the existence of a weak solution $(\phi, \psi)$ with the regularity stated in (4.15). In particular, it is shown in [34, (4.34)-(4.40)] that

$$
\begin{aligned}
& \|(\phi, \psi)\|_{L^{\infty}\left(0, T ; V_{\gamma}^{1}\right)}^{2}+\|(\phi, \psi)\|_{H^{1}\left(0, T ;\left(H^{1}(\Omega)\right)^{*} \times L^{2}(\Gamma)\right)}^{2} \\
& +\|\widehat{\mu}\|_{L^{2}\left(0, T ; H^{1}(\Omega)\right)}^{2}+\sigma\left\|\partial_{t} \phi\right\|_{L^{2}\left(0, T ; L^{2}(\Omega)\right)}^{2} \\
& \leq C\left(\left\|\left(\phi_{0}, \psi_{0}\right)\right\|_{V_{\gamma}^{1}}^{2}+\left\|l_{1}\right\|_{L^{2}\left(0, T ;\left(H^{1}(\Omega)\right)^{*}\right)}^{2}+\left\|l_{2}\right\|_{L^{2}\left(0, T ; L^{2}(\Omega)\right)}^{2}+\left\|l_{3}\right\|_{L^{2}\left(0, T ; L^{2}(\Gamma)\right)}^{2}\right),
\end{aligned}
$$

for some constant $C>0$ independent of $\sigma \in[0,1]$. The regularity part stated in (4.16) is obtained on account of Lemma 4.6, estimate (4.18) and the fact that, for almost any $t \in(0, T),(\phi(t), \psi(t))$ is also a weak solution to the elliptic boundary value problem

$$
-\Delta \phi=\widehat{\mu}-\sigma \partial_{t} \phi-l_{2}, \text { in } \Omega \times(0, \infty), \quad-\gamma A_{\tau} \psi+\partial_{n} \phi+\zeta \psi=-\partial_{t} \psi+l_{3}, \text { on } \Gamma \times(0, \infty) .
$$

The uniqueness of the weak solution $(\phi, \psi)$ is also shown in [34, Section 3] and applies to all cases $\sigma \in[0,1]$. This completes the proof. 


\section{Proof of Theorem 3.2}

The main goal of this section is to prove the existence of at least one weak energy solution to the original problem by passing to the limit as $\epsilon, \sigma \rightarrow 0$ in a regularized version of system (1.1)-(1.10) which admits a sufficiently smooth approximate solution $\left(u_{\epsilon, \sigma}, \phi_{\epsilon, \sigma}, \psi_{\epsilon, \sigma}\right)$. The existence of such solutions will be provided by a fixed point argument. Then, we will derive additional uniform estimates for the solutions $\left(u_{\epsilon, \sigma}, \phi_{\epsilon, \sigma}, \psi_{\epsilon, \sigma}\right)$ as $\epsilon, \sigma \rightarrow 0$. This program is divided into three main steps.

Step 1. The approximating problem $\mathbf{P}_{\epsilon, \sigma}$. We start with the following preliminary points.

- We let $J_{\epsilon}, K_{\epsilon}$ be mollifiers acting on functions from $H^{m}(\Omega)$ (as well as from $\mathbb{H}^{m}$ ) and $H^{m}(\Gamma)$, respectively. These mollifiers obey the stated properties of Lemma 4.1.

- We take, for $\epsilon \in(0,1)$, smooth functions $f_{\epsilon} \rightarrow f, g_{\epsilon} \rightarrow f$ uniformly on compact intervals of $\mathbb{R}$, with the following properties:

$$
\left|f_{\epsilon}^{\prime}(s)\right| \leq c_{f, \epsilon},\left|g_{\epsilon}^{\prime}(s)\right| \leq c_{g, \epsilon}, \quad \text { for all } s \in \mathbb{R}
$$

for some positive constants $c_{f, \epsilon}, c_{g, \epsilon}$ which may behave like $\epsilon^{-m}$ as $\epsilon \rightarrow 0$, for some $m \in \mathbb{N}$. Setting $F_{\epsilon}(s)=\int_{0}^{s} f_{\epsilon}(\zeta) d \zeta$ and $G_{\epsilon}(s)=\int_{0}^{s} g_{\epsilon}(\zeta) d \zeta$, we also assume

$$
\limsup _{\epsilon \rightarrow 0} F_{\epsilon}(s)=F(s), \limsup _{\epsilon \rightarrow 0} G_{\epsilon}(s)=G(s), \quad \text { for all } s \in \mathbb{R} .
$$

In particular, we observe that (5.1) implies at most a linear growth of $f_{\epsilon}, g_{\epsilon}$ as $|s| \rightarrow \infty$. Such new functions $f_{\epsilon}, g_{\epsilon} \in C^{2}(\mathbb{R})$ can be easily realized by following the constructions given in [17, pp. 2382] (see also [34, pp. 79]).

- We insert an artificial viscosity $\sigma \in(0,1)$ into the problem by adding the viscous term $\sigma \partial_{t} \phi$ in the definition of the chemical potential (see (5.6)). Its effect on the regularized problem for $\epsilon>0$ turns out to be quite minimal in the sense that all the estimates proven below will be independent of any $\sigma \geq 0$. However, the presence of the viscous term allows us to gain additional regularity for $\phi$ and this information will be crucial in the limit procedure as $(\epsilon, \sigma) \rightarrow(0,0)$.

Our approximated problem $\mathbf{P}_{\epsilon, \sigma}, \epsilon, \sigma \in(0,1)$, consists in finding a function $\left(u_{\epsilon, \sigma}, \phi_{\epsilon, \sigma}, \psi_{\epsilon, \sigma}\right)$ which has the regularity stated in $(2.3)-(2.4)$ and, additionally,

$$
u_{\epsilon, \sigma} \in H^{1}\left(0, T ;\left(\mathbb{H}^{1}\right)^{*}\right), \quad\left(\phi_{\epsilon, \sigma}, \psi_{\epsilon, \sigma}\right) \in H^{1}\left(0, T ; L^{2}(\Omega) \times L^{2}(\Gamma)\right),
$$

such that

$$
\begin{aligned}
& \left(\partial_{t} u_{\epsilon, \sigma}(t), v\right)+a_{0}\left(u_{\epsilon, \sigma}(t), v\right)+b_{0}\left(J_{\epsilon} u_{\epsilon, \sigma}(t), u_{\epsilon, \sigma}(t), v\right) \\
& =b_{1}\left(v, J_{\epsilon} \phi_{\epsilon, \sigma}(t), \mu_{\epsilon, \sigma}(t)\right)+\left(\mathcal{L}_{\epsilon}\left(\psi_{\epsilon, \sigma}(t)\right) \nabla_{\tau}\left(K_{\epsilon} \psi_{\epsilon, \sigma}\right)(t), v_{\tau}\right)_{\Gamma}+\left(h_{\epsilon}(t), v\right),
\end{aligned}
$$

holds for all $v \in \mathbb{H}^{1}$, for almost any $t \in(0, T)$ and for every fixed $T>0$ and

$$
\begin{gathered}
\left(\partial_{t} \phi_{\epsilon, \sigma}(t), \varphi\right)+\left(\nabla \mu_{\epsilon, \sigma}(t), \nabla \varphi\right)+b_{1}\left(u_{\epsilon, \sigma}(t), J_{\epsilon} \phi_{\epsilon, \sigma}(t), \varphi\right)=0 \\
\left(\partial_{t} \psi_{\epsilon, \sigma}(t), \eta\right)_{\Gamma}+b_{\Gamma}\left(u_{\epsilon, \sigma}(t),\left(K_{\epsilon} \psi_{\epsilon, \sigma}\right)(t), \eta\right)+\left(\mathcal{L}_{\epsilon}\left(\psi_{\epsilon, \sigma}(t)\right), \eta\right)_{\Gamma}=0
\end{gathered}
$$

hold for all $\varphi \in H^{1}(\Omega), \eta \in L^{2}(\Gamma)$ and for almost any $t \in(0, T)$, with

$$
\begin{aligned}
\mu_{\epsilon, \sigma} & =-\Delta \phi_{\epsilon, \sigma}+\sigma \partial_{t} \phi_{\epsilon, \sigma}+f_{\epsilon}\left(\phi_{\epsilon, \sigma}\right) \text { a.e. in } \Omega \times(0, T), \\
\mathcal{L}_{\epsilon}\left(\psi_{\epsilon, \sigma}\right) & =-\gamma A_{\tau} \psi_{\epsilon, \sigma}+\partial_{n} \phi_{\epsilon, \sigma}+\zeta \psi_{\epsilon, \sigma}+g_{\epsilon}\left(\psi_{\epsilon, \sigma}\right) \text { a.e. on } \Gamma \times(0, T) .
\end{aligned}
$$

Furthermore, the initial conditions are satisfied:

$$
u_{\epsilon, \sigma}(0)=u_{0}, \phi_{\epsilon, \sigma}(0)=\phi_{0}, \psi_{\epsilon, \sigma}(0)=\psi_{0} .
$$

Note that, in (5.3), the function $h$ has been replaced by $J_{\epsilon} h$.

Step 2. Existence of solutions for $\mathbf{P}_{\epsilon, \sigma}$. Let $T>0$ be given and set

$$
\left\{\begin{array}{l}
X_{T}:=\left\{u \in L^{\infty}(0, T ; \mathbb{H}) \cap H^{1}\left(0, T ;\left(\mathbb{H}^{1}\right)^{*}\right) \cap L^{2}\left(0, T ; \mathbb{H}^{1}\right): u_{\mid t=0}=u_{0}\right\}, \\
Y_{T}:=\left\{(\phi, \psi) \in L^{\infty}\left(0, T ; V_{\gamma}^{1}\right) \cap H^{1}\left(0, T ; V_{\gamma}^{0}\right) \cap L^{2}\left(0, T ; V_{\gamma}^{2}\right):(\phi, \psi)_{\mid t=0}=\left(\phi_{0}, \psi_{0}\right)\right\} .
\end{array}\right.
$$

For $\epsilon, \sigma \in(0,1)$ and for each $\left(v_{\epsilon, \sigma}, \varphi_{\epsilon, \sigma}, \eta_{\epsilon, \sigma}\right) \in X_{T} \times Y_{T}$, let us consider the problem of finding $\left(u_{\epsilon, \sigma}, \phi_{\epsilon, \sigma}, \psi_{\epsilon, \sigma}\right) \in X_{T} \times Y_{T}$ solution to

$$
\left\langle\partial_{t} u_{\epsilon, \sigma}(t), v\right\rangle+a_{0}\left(u_{\epsilon, \sigma}(t), v\right)+b_{0}\left(J_{\epsilon} v_{\epsilon, \sigma}(t), v_{\epsilon, \sigma}(t), v\right)
$$




$$
=b_{1}\left(v,\left(J_{\epsilon} \varphi_{\epsilon, \sigma}\right)(t), \widehat{\mu}_{\epsilon, \sigma}(t)\right)+\left(\widehat{\mathcal{L}}_{\epsilon}\left(\eta_{\epsilon, \sigma}(t)\right) \nabla_{\tau}\left(K_{\epsilon} \eta_{\epsilon, \sigma}\right)(t), v_{\tau}\right)_{\Gamma}+\left(h_{\epsilon}(t), v\right),
$$

for all $v \in \mathbb{H}^{1}$ and for almost any $t \in(0, T)$, and

$$
\begin{gathered}
\left\langle\partial_{t} \phi_{\epsilon, \sigma}(t), \varphi\right\rangle+\left(\nabla \widehat{\mu}_{\epsilon, \sigma}(t), \nabla \varphi\right)+b_{1}\left(v_{\epsilon, \sigma}(t),\left(J_{\epsilon} \varphi_{\epsilon, \sigma}\right)(t), \varphi\right)=0, \\
\left(\partial_{t} \psi_{\epsilon, \sigma}(t), \eta\right)_{\Gamma}+b_{\Gamma}\left(v_{\epsilon, \sigma}(t),\left(K_{\epsilon} \eta_{\epsilon, \sigma}\right)(t), \eta\right)+\left(\widehat{\mathcal{L}}_{\epsilon}\left(\psi_{\epsilon, \sigma}(t)\right), \eta\right)_{\Gamma}=-\left(g_{\epsilon}\left(\eta_{\epsilon, \sigma}\right), \eta\right)_{\Gamma},
\end{gathered}
$$

for all $\varphi \in H^{1}(\Omega), \eta \in L^{2}(\Gamma)$ and for almost any $t \in(0, T)$, with

$$
\begin{aligned}
\widehat{\mu}_{\epsilon, \sigma} & =-\Delta \varphi_{\epsilon, \sigma}+\sigma \partial_{t} \varphi_{\epsilon, \sigma}+f_{\epsilon}\left(\varphi_{\epsilon, \sigma}\right), \text { a.e. in } \Omega \times(0, T), \\
\widehat{\mathcal{L}}_{\epsilon}\left(\eta_{\epsilon, \sigma}\right) & =-\gamma A_{\tau} \eta_{\epsilon, \sigma}+\partial_{n} \varphi_{\epsilon, \sigma}+\zeta \eta_{\epsilon, \sigma}, \text { a.e. on } \Gamma \times(0, T) .
\end{aligned}
$$

First, we observe that, if $v_{\epsilon, \sigma} \in X_{T}$, then, setting $\varphi \equiv 1$ in (5.10), we find $\left\langle\partial_{t} \phi_{\epsilon, \sigma}(t)\right\rangle=0$ almost everywhere $t \in(0, T)$, i.e., mass is conserved for the component $\phi_{\epsilon, \sigma}$. Also, on account of $\left(\varphi_{\epsilon, \sigma}, \eta_{\epsilon, \sigma}\right) \in Y_{T}$, we have $\widehat{\mu}_{\epsilon, \sigma} \in L^{2}\left(0, T ; L^{2}(\Omega)\right)$ and $\widehat{\mathcal{L}}_{\epsilon}\left(\eta_{\epsilon, \sigma}\right) \in L^{2}\left(0, T ; L^{2}(\Gamma)\right)$. By Lemma 4.1, observe that $J_{\epsilon} \varphi_{\epsilon, \sigma} \in L^{\infty}\left(0, T ; H^{3}(\Omega)\right), J_{\epsilon} v_{\epsilon, \sigma} \in L^{\infty}\left(0, T ; \mathbb{H}^{2}\right)$ and $K_{\epsilon} \eta_{\epsilon, \sigma} \in L^{\infty}\left(0, T ; H^{3}(\Gamma)\right)$. Thus we have

$$
h_{1}:=h_{\epsilon}-B_{0}\left(J_{\epsilon} v_{\epsilon, \sigma}, v_{\epsilon, \sigma}\right)+\nabla\left(J_{\epsilon} \varphi_{\epsilon, \sigma}\right) \widehat{\mu}_{\epsilon, \sigma} \in L^{2}\left(0, T ; \mathbb{L}^{2}(\Omega)\right)
$$

and

$$
h_{2}:=\widehat{\mathcal{L}}_{\epsilon}\left(\eta_{\epsilon, \sigma}\right) \nabla_{\tau}\left(K_{\epsilon} \eta_{\epsilon, \sigma}\right) \in L^{2}\left(0, T ; L^{4 / 3}(\Gamma)^{N-1}\right) .
$$

It is also not difficult to check that

$$
\begin{aligned}
& l_{1}:=-B_{1}\left(v_{\epsilon, \sigma}, J_{\epsilon} \varphi_{\epsilon, \sigma}\right) \in L^{2}\left(0, T ; L^{2}(\Omega)\right), \\
& l_{2}:=f_{\epsilon}\left(\varphi_{\epsilon, \sigma}\right) \in L^{2}\left(0, T ; L^{2}(\Gamma)\right), \\
& l_{3}:=-B_{\Gamma}\left(v_{\epsilon, \sigma}, K_{\epsilon} \eta_{\epsilon, \sigma}\right)-g_{\epsilon}\left(\eta_{\epsilon, \sigma}\right) \in L^{2}\left(0, T ; L^{2}(\Gamma)\right),
\end{aligned}
$$

with $\left\langle l_{1}(t)\right\rangle \equiv 0$, for all $t>0$, thanks to assumption (5.1) and the fact that $\left(v_{\epsilon, \sigma}, \varphi_{\epsilon, \sigma}, \eta_{\epsilon, \sigma}\right) \in$ $X_{T} \times Y_{T}$. Thus, on account of Theorems 4.9 and 4.10, problem (5.9) has a unique solution $u_{\epsilon, \sigma} \in X_{T}$, while problem (5.10)-(5.13) admits a unique solution $\left(\phi_{\epsilon, \sigma}, \psi_{\epsilon, \sigma}\right) \in Y_{T}$. Henceforth, the mapping

$$
S: X_{T} \times Y_{T} \rightarrow X_{T} \times Y_{T},\left(v_{\epsilon, \sigma}, \varphi_{\epsilon, \sigma}, \eta_{\epsilon, \sigma}\right) \mapsto\left(u_{\epsilon, \sigma}, \phi_{\epsilon, \sigma}, \psi_{\epsilon, \sigma}\right)
$$

is well defined. The existence of a solution to problem $\mathbf{P}_{\epsilon, \sigma}$ on some interval $(0, T)$ with $T=$ $T_{\epsilon, \sigma}>0$ will be sought as the unique fixed point of the map $S$.

Next, we show that $S$ maps a bounded subset of $X_{T} \times Y_{T}$ into itself if $T$ is small enough. To this end, we introduce the subset $Z_{T}:=X_{T} \times Y_{T}$ which consists of functions $\left(v_{\epsilon, \sigma}, \varphi_{\epsilon, \sigma}, \eta_{\epsilon, \sigma}\right) \in Z_{T}$ such that

$$
\left\|v_{\epsilon, \sigma}\right\|_{X_{T}}^{2} \leq M_{1},\left\|\left(\varphi_{\epsilon, \sigma}, \eta_{\epsilon, \sigma}\right)\right\|_{Y_{T}}^{2} \leq M_{2}
$$

where the positive constants $M_{i}(i=1,2)$ will be specified below. Then we prove that $S: Z_{T} \rightarrow Z_{T}$ is a contraction, provided that $T=T_{\epsilon, \sigma}>0$ is sufficiently small.

Step 2.1 (The mapping $S: Z_{T} \rightarrow Z_{T}$ is well defined) Let $\left(v_{\epsilon, \sigma}, \varphi_{\epsilon, \sigma}, \eta_{\epsilon, \sigma}\right) \in Z_{T}$ and

$$
\left(u_{\epsilon, \sigma}, \phi_{\epsilon, \sigma}, \psi_{\epsilon, \sigma}\right)=S\left(v_{\epsilon, \sigma}, \varphi_{\epsilon, \sigma}, \eta_{\epsilon, \sigma}\right) .
$$

We start with an estimate for $u_{\epsilon, \sigma}$, taking advantage of (4.7). From (5.9), we have

$$
\begin{aligned}
& \quad \underset{t \in(0, T)}{\operatorname{essup}}\left|u_{\epsilon, \sigma}(t)\right|^{2}+\int_{0}^{T}\left(\left\|u_{\epsilon, \sigma}(s)\right\|^{2}+\left\|\partial_{t} u_{\epsilon, \sigma}(s)\right\|_{\left(\mathbb{H}^{1}\right)^{*}}^{2}\right) d s \\
& \leq \quad C_{1}\left|u_{0}\right|^{2}+C_{1} \int_{0}^{T}\left(\left\|B_{0}\left(J_{\epsilon} v_{\epsilon, \sigma}, v_{\epsilon, \sigma}\right)\right\|_{\left(\mathbb{H}^{1}\right)^{*}}^{2}+\left\|\widehat{\mu}_{\epsilon, \sigma} \nabla\left(J_{\epsilon} \varphi_{\epsilon, \sigma}\right)\right\|_{\left(\mathbb{H}^{1}\right)^{*}}^{2}+\left\|h_{\epsilon}\right\|_{\left(\mathbb{H}^{1}\right)^{*}}^{2}\right) d s \\
& +C_{1} \int_{0}^{T}\left\|\widehat{\mathcal{L}}_{\epsilon}\left(\eta_{\epsilon, \sigma}\right) \nabla_{\tau}\left(K_{\epsilon} \eta_{\epsilon, \sigma}\right)\right\|_{4 / 3, \Gamma}^{2} d s .
\end{aligned}
$$


Note that each term $\left(J_{\epsilon} v_{\epsilon, \sigma}\right)_{i} \partial_{i}\left(v_{\epsilon, \sigma}\right)_{j}(1 \leq i, j \leq \mathrm{N})$ is a product of distributions in $H^{2}(\Omega)$ and $\left(H^{1}(\Omega)\right)^{*}$. Therefore, it is bounded in $\left(H^{1}(\Omega)\right)^{*}$ since $H^{2}(\Omega) \hookrightarrow C^{0, \vartheta}(\bar{\Omega})$. Thus, it follows from Lemma 4.1-(iv) that

$$
\begin{aligned}
& \underset{t \in(0, T)}{e s s \sup }\left|u_{\epsilon, \sigma}(t)\right|^{2}+\int_{0}^{T}\left(\left\|u_{\epsilon, \sigma}(s)\right\|^{2}+\left\|\partial_{t} u_{\epsilon, \sigma}(s)\right\|_{\left(\mathbb{H}^{1}\right)^{*}}^{2}\right) d s \\
& \leq C_{1}\left(\left|u_{0}\right|^{2}+\|h\|_{L^{2}\left(0, T ;\left(\mathbb{H}^{1}\right)^{*}\right)}^{2}\right)+C_{2} \int_{0}^{T}\left(\left\|J_{\epsilon} v_{\epsilon, \sigma}\right\|_{H^{2}}^{2}\left|v_{\epsilon, \sigma}\right|^{2}+\left\|\widehat{\mu}_{\epsilon, \sigma}\right\|_{2}^{2}\left\|\nabla\left(J_{\epsilon} \varphi_{\epsilon, \sigma}\right)\right\|_{H^{1}}^{2}\right) d s \\
& +C_{2} \int_{0}^{T}\left\|\widehat{\mathcal{L}}_{\epsilon}\left(\eta_{\epsilon, \sigma}\right)\right\|_{2, \Gamma}^{2}\left\|\nabla_{\tau}\left(K_{\epsilon} \eta_{\epsilon, \sigma}\right)\right\|_{H^{2}(\Gamma)}^{2} d s \\
& \leq C_{1}\left(\left|u_{0}\right|^{2}+\|h\|_{L^{2}\left(0, T ;\left(\mathbb{H}^{1}\right)^{*}\right)}^{2}\right)+C_{3}\left(\frac{T M_{1}^{2}}{\epsilon^{4}}+\frac{M_{2}^{2}}{\epsilon^{2}}+\frac{M_{2}^{2}}{\epsilon^{6}}\right)
\end{aligned}
$$

owing to (5.15) and the fact that $K_{\epsilon} \eta_{\epsilon, \sigma} \in H^{3}(\Gamma)$. Here the constant $C_{3}>0$ is also independent of $\gamma>0$ and $\sigma>0$ (actually, this applies to all constants $C_{i}>0$ in the proof below). Then, we choose

$$
M_{1} \geq 2 C_{1}\left(\left|u_{0}\right|^{2}+\|h\|_{L^{2}\left(0, T ;\left(\mathbb{H}^{1}\right)^{*}\right)}^{2}\right)+2 C_{3}\left(\frac{M_{2}^{2}}{\epsilon^{2}}+\frac{M_{2}^{2}}{\epsilon^{6}}\right),
$$

where $M_{2}$ will be chosen below (see (5.22)), and $T=T_{\epsilon, \sigma}>0$ such that

$$
T \leq \frac{\epsilon^{4}}{2 C_{3} M_{1}}
$$

Henceforth, $u_{\epsilon, \sigma} \in X_{T}$ whenever $T>0$ and $M_{1}>0$ satisfy (5.19), (5.18). Let us now estimate $\left(\phi_{\epsilon, \sigma}, \psi_{\epsilon, \sigma}\right)$ (see problem (5.9)-(5.13)). Using (4.17), we get

$$
\begin{aligned}
& \text { ess sup }\left\|\left(\phi_{\epsilon, \sigma}(t), \psi_{\epsilon, \sigma}(t)\right)\right\|_{V_{\gamma}^{1}}^{2}+\left\|\left(\phi_{\epsilon, \sigma}, \psi_{\epsilon, \sigma}\right)\right\|_{H^{1}\left(0, T ;\left(H^{1}(\Omega)\right)^{*} \times L^{2}(\Gamma)\right)}^{2} \\
& +\sigma\left\|\partial_{t} \phi_{\epsilon, \sigma}\right\|_{L^{2}\left(0, T ; L^{2}(\Omega)\right)}^{2}+\left\|\left(\phi_{\epsilon, \sigma}, \psi_{\epsilon, \sigma}\right)\right\|_{L^{2}\left(0, T ; V_{\gamma}^{2}\right)}^{2} \\
& \leq C_{4}\left(\left\|\left(\phi_{0}, \psi_{0}\right)\right\|_{V_{\gamma}^{1}}^{2}+\left\|B_{1}\left(v_{\epsilon, \sigma}, J_{\epsilon} \varphi_{\epsilon, \sigma}\right)\right\|_{L^{2}\left(0, T ; L^{2}(\Omega)\right)}^{2}+\left\|f_{\epsilon}\left(\varphi_{\epsilon, \sigma}\right)\right\|_{L^{2}\left(0, T ; L^{2}(\Omega)\right)}^{2}\right) \\
& +C_{4}\left(\left\|B_{\Gamma}\left(v_{\epsilon, \sigma}, K_{\epsilon} \eta_{\epsilon, \sigma}\right)\right\|_{L^{2}\left(0, T ; L^{2}(\Gamma)\right)}^{2}+\left\|g_{\epsilon}\left(\eta_{\epsilon, \sigma}\right)\right\|_{L^{2}\left(0, T ; L^{2}(\Gamma)\right)}^{2}\right) .
\end{aligned}
$$

Thus, by applying Lemma 4.1-(iv) and exploiting (5.1) together with the regularity of $J_{\epsilon} \varphi_{\epsilon, \sigma}$ and $K_{\epsilon} \eta_{\epsilon, \sigma}$, we infer

$$
\begin{aligned}
& \underset{t \in(0, T)}{\operatorname{ess} \sup }\left\|\left(\phi_{\epsilon, \sigma}(t), \psi_{\epsilon, \sigma}(t)\right)\right\|_{V_{\gamma}^{1}}^{2}+\left\|\left(\phi_{\epsilon, \sigma}, \psi_{\epsilon, \sigma}\right)\right\|_{H^{1}\left(0, T ;\left(H^{1}(\Omega)\right)^{*} \times L^{2}(\Gamma)\right)}^{2} \\
& +\sigma\left\|\partial_{t} \phi_{\epsilon, \sigma}\right\|_{L^{2}\left(0, T ; L^{2}(\Omega)\right)}^{2}+\left\|\left(\phi_{\epsilon, \sigma}, \psi_{\epsilon, \sigma}\right)\right\|_{L^{2}\left(0, T ; V_{\gamma}^{2}\right)}^{2} \\
& \leq C_{5}\left(1+\left\|\left(\phi_{0}, \psi_{0}\right)\right\|_{V_{\gamma}^{1}}^{2}\right)+C_{6} \int_{0}^{T}\left\|J_{\epsilon} \varphi_{\epsilon, \sigma}\right\|_{H^{3}}^{2}\left|v_{\epsilon, \sigma}\right|^{2} d s \\
& +C_{6} \int_{0}^{T}\left\|\nabla_{\tau}\left(K_{\epsilon} \eta_{\epsilon, \sigma}\right)\right\|_{H^{3}(\Gamma)}^{2}\left\|\left(v_{\epsilon, \sigma}\right)_{\tau}\right\|_{2, \Gamma}^{2} d s \\
& \leq C_{5}\left(1+\left\|\left(\phi_{0}, \psi_{0}\right)\right\|_{V_{\gamma}^{1}}^{2}\right)+C_{7} \frac{M_{1} M_{2} T}{\epsilon^{2}}+C_{7}\left(\frac{\widetilde{\delta} M_{1} M_{2}}{\epsilon^{3}}+\frac{\widetilde{\delta}^{-\alpha} T M_{1} M_{2}}{\epsilon^{3}}\right),
\end{aligned}
$$

for every $\widetilde{\delta} \in(0,1)$ and some $\alpha>0$. Here $C_{5}>0$ also depends on the constants $c_{f, \epsilon}$ and $c_{g, \epsilon}$ from (5.1), but is independent of $\gamma, \sigma>0$. The last bound on the right-hand side of (5.20) has been 
deduced as follows:

$$
\begin{aligned}
& \int_{0}^{T}\left\|\nabla_{\tau}\left(K_{\epsilon} \eta_{\epsilon, \sigma}\right)\right\|_{H^{3}}^{2}\left\|\left(v_{\epsilon, \sigma}\right)_{\tau}\right\|_{2, \Gamma}^{2} d s \\
& \leq \widetilde{\delta} \int_{0}^{T}\left\|K_{\epsilon} \eta_{\epsilon, \sigma}\right\|_{H^{3}(\Gamma)}^{2}\left\|v_{\epsilon, \sigma}\right\|^{2} d s+\widetilde{\delta}^{-\alpha} \int_{0}^{T}\left\|K_{\epsilon} \eta_{\epsilon, \sigma}\right\|_{H^{3}(\Gamma)}^{2}\left|v_{\epsilon, \sigma}\right|^{2} d s \\
& \leq \frac{C_{7}}{\epsilon^{3}}\left(\widetilde{\delta} M_{1} M_{2}+\widetilde{\delta}^{-\alpha} T M_{1} M_{2}\right)
\end{aligned}
$$

on account of Lemma 4.3) and (5.15). Therefore, (5.20) entails

$$
\begin{aligned}
\left\|\left(\phi_{\epsilon, \sigma}, \psi_{\epsilon, \sigma}\right)\right\|_{Y_{T}}^{2} & \leq C_{5}\left(1+\left\|\left(\phi_{0}, \psi_{0}\right)\right\|_{V_{\gamma}^{1}}^{2}\right) \\
& +C_{7} T\left(\frac{M_{1} M_{2}}{\epsilon^{2}}+\frac{\widetilde{\delta}^{-\alpha} M_{1} M_{2}}{\epsilon^{3}}\right)+\widetilde{\delta} \frac{C_{7} M_{1} M_{2}}{\epsilon^{3}} .
\end{aligned}
$$

Then, choosing

$$
M_{2} \geq 3 C_{5}\left(1+\left\|\left(\phi_{0}, \psi_{0}\right)\right\|_{V_{\gamma}^{1}}^{2}\right) \text { and } \widetilde{\delta}<\min \left\{\frac{1}{2}, \frac{\epsilon^{3}}{3 C_{7} M_{1}}\right\}
$$

with a time $T=T_{\epsilon, \sigma}>0$ satisfying

$$
C_{7} T\left(\frac{M_{1}}{\epsilon^{2}}+\frac{\widetilde{\delta}^{-\alpha} M_{1}}{\epsilon^{3}}\right) \leq \frac{1}{3}
$$

we infer that $\left(\phi_{\epsilon, \sigma}, \psi_{\epsilon, \sigma}\right) \in Y_{T}$ as well. Thus we can find $T>0$ small enough and $M_{1}, M_{2}>0$ large enough such that $S$ maps $Z_{T}$ into itself.

Remark 5.1. Note that $T>0$ satisfying (5.19) and (5.23) is independent of $\sigma \geq 0$.

Step 2.2 $\left(S\right.$ is a contraction on $Z_{T}$ ) Let us now show that, possibly choosing $T=T_{\epsilon}$ smaller than the one which satisfies (5.19) and (5.23), $S: Z_{T} \rightarrow Z_{T}$ is a contraction with respect to the metric induced by the norm of $X_{T} \times Y_{T}$ which makes $Z_{T}$ complete. For the sake of simplicity, we will drop the dependence on $\sigma$ from the notation of functions, owing to the fact that all estimates below are independent of $\sigma \in[0,1]$. Let $\left(v_{j \epsilon}, \varphi_{j \epsilon}, \eta_{j \epsilon}\right) \in Z_{T}$ for $j=1,2$ and $\left(u_{j \epsilon}, \phi_{j \epsilon}, \psi_{j \epsilon}\right)=S\left(v_{j \epsilon}, \varphi_{j \epsilon}, \eta_{j \epsilon}\right)$. Then, set $u_{\epsilon}:=u_{1 \epsilon}-u_{2 \epsilon}, \phi_{\epsilon}:=\phi_{1 \epsilon}-\phi_{2 \epsilon}, \psi_{\epsilon}:=\psi_{1 \epsilon}-\psi_{2 \epsilon}$ and $v_{\epsilon}:=v_{1 \epsilon}-v_{2 \epsilon}, \varphi_{\epsilon}:=\varphi_{1 \epsilon}-\varphi_{2 \epsilon}$, $\eta_{\epsilon}:=\eta_{1 \epsilon}-\eta_{2 \epsilon}$. Thus, $u_{\epsilon}$ is a weak solution to the problem

$$
\left\{\begin{array}{l}
\partial_{t} u_{\epsilon}+\operatorname{div}\left(2 \nu D\left(u_{\epsilon}\right)\right)=-B_{0}\left(J_{\epsilon} v_{\epsilon}, v_{1 \epsilon}\right)-B_{0}\left(J_{\epsilon} v_{2 \epsilon}, v_{\epsilon}\right)+\bar{\mu}_{1 \epsilon} \nabla\left(J_{\epsilon} \varphi_{1 \epsilon}\right)-\bar{\mu}_{2 \epsilon} \nabla\left(J_{\epsilon} \varphi_{2 \epsilon}\right) \\
2 \nu\left(D\left(u_{\epsilon}\right) \cdot n\right)_{\tau}+\beta u_{\epsilon}=\overline{\mathcal{L}}_{\epsilon}\left(\eta_{\epsilon}\right) \nabla_{\tau}\left(K_{\epsilon} \eta_{1 \epsilon}\right)+\mathcal{L}_{\epsilon}\left(\eta_{2 \epsilon}\right) \nabla_{\tau}\left(K_{\epsilon} \eta_{\epsilon}\right) \\
u_{\epsilon}(0)=0
\end{array}\right.
$$

with $\mathcal{L}_{\epsilon}(\eta)=-\gamma A_{\tau} \eta+\partial_{n} \varphi+\zeta \eta+g_{\epsilon}(\eta)$ and

$$
\bar{\mu}_{i \epsilon}:=-\Delta \varphi_{i \epsilon}+\sigma \partial_{t} \varphi_{i \epsilon}+f_{\epsilon}\left(\varphi_{i \epsilon}\right), \overline{\mathcal{L}}_{\epsilon}\left(\eta_{\epsilon}\right):=-\gamma A_{\tau} \eta_{i \epsilon}+\partial_{n} \varphi_{i \epsilon}+\zeta \eta_{i \epsilon}+g_{\epsilon}\left(\eta_{1 \epsilon}\right)-g_{\epsilon}\left(\eta_{2 \epsilon}\right) .
$$

Furthermore, $\left(\phi_{\epsilon}, \psi_{\epsilon}\right)$ is a weak solution to the problem

$$
\left\{\begin{array}{l}
\partial_{t} \phi_{\epsilon}+A_{N} \bar{\mu}_{\epsilon}=-B_{1}\left(v_{\epsilon}, J_{\epsilon} \varphi_{1 \epsilon}\right)-B_{1}\left(v_{2 \epsilon}, J_{\epsilon} \varphi_{\epsilon}\right), \\
\partial_{t} \psi_{\epsilon}+\widehat{\mathcal{L}}_{\epsilon}\left(\psi_{\epsilon}\right)=-B_{\Gamma}\left(v_{\epsilon}, K_{\epsilon} \eta_{1 \epsilon}\right)-B_{\Gamma}\left(v_{2 \epsilon}, K_{\epsilon} \eta_{\epsilon}\right)+g_{\epsilon}\left(\eta_{2 \epsilon}\right)-g_{\epsilon}\left(\eta_{1 \epsilon}\right), \\
(\phi(0), \psi(0))=(0,0),
\end{array}\right.
$$

where $\widehat{\mathcal{L}}_{\epsilon}(\cdot)$ is given by $(5.13)$ and

$$
\bar{\mu}_{\epsilon}:=-\Delta \phi_{\epsilon}+\sigma \partial_{t} \phi_{\epsilon}+f_{\epsilon}\left(\varphi_{1 \epsilon}\right)-f_{\epsilon}\left(\varphi_{2 \epsilon}\right) .
$$

Notice that $\left\langle\phi_{\epsilon}(t)\right\rangle=0$, since $\left\langle\phi_{i \epsilon}(t)\right\rangle=\left\langle\phi_{0}\right\rangle, i=1,2$, for $t>0$. We will rely once again on the results of Theorems 4.9 and 4.10 . We start with an estimate of $u_{\epsilon}$ in $X_{T}$. Pairing the first equation of (5.24) with $u_{\epsilon}$ in the scalar product of $\mathbb{H}$, we have

$$
\frac{1}{2} \underset{t \in(0, T)}{e s s \sup _{\epsilon}}\left|u_{\epsilon}(t)\right|^{2}+\int_{0}^{T} a_{0}\left(u_{\epsilon}(t), u_{\epsilon}(t)\right)+\left\|\partial_{t} u_{\epsilon}(t)\right\|_{\left(\mathbb{H}^{1}\right)^{*}}^{2} d t \leq \sum_{i=-1}^{8} \int_{0}^{T} I_{i}(t) d t
$$


where we have set

$$
\begin{cases}I_{-1}:=\sigma\left(\partial_{t} \varphi_{\epsilon} \nabla\left(J_{\epsilon} \varphi_{1 \epsilon}\right), u_{\epsilon}\right) & I_{0}:=\sigma\left(\partial_{t} \varphi_{2 \epsilon} \nabla\left(J_{\epsilon} \varphi_{\epsilon}\right), u_{\epsilon}\right), \\ I_{1}:=-b_{0}\left(J_{\epsilon} v_{\epsilon}, v_{1 \epsilon}, u_{\epsilon}\right), & I_{2}:=-b_{0}\left(J_{\epsilon} v_{2 \epsilon}, v_{\epsilon}, u_{\epsilon}\right), \\ I_{3}:=\left(\left(f_{\epsilon}\left(\varphi_{1 \epsilon}\right)-f_{\epsilon}\left(\varphi_{2 \epsilon}\right)\right) \nabla\left(J_{\epsilon} \varphi_{1 \epsilon}\right), u_{\epsilon}\right), & I_{4}:=\left(f_{\epsilon}\left(\varphi_{2 \epsilon}\right) \nabla\left(J_{\epsilon} \varphi_{\epsilon}\right), u_{\epsilon}\right), \\ I_{5}:=\left(-\Delta \varphi_{\epsilon} \nabla\left(J_{\epsilon} \varphi_{1 \epsilon}\right), u_{\epsilon}\right), & I_{6}:=\left(-\Delta \varphi_{2 \epsilon} \nabla\left(J_{\epsilon} \varphi_{\epsilon}\right), u_{\epsilon}\right), \\ I_{7}:=\left(\overline{\mathcal{L}}_{\epsilon}\left(\eta_{\epsilon}\right) \nabla_{\tau}\left(K_{\epsilon} \eta_{1 \epsilon}\right), u_{\epsilon \tau}\right)_{\Gamma}, & I_{8}:=\left(\mathcal{L}_{\epsilon}\left(\eta_{2 \epsilon}\right) \nabla_{\tau}\left(K_{\epsilon} \eta_{\epsilon}\right), u_{\epsilon \tau}\right)_{\Gamma} .\end{cases}
$$

We now estimate all the terms $I_{-1}, I_{0}, \ldots, I_{8}$. First, by elementary Hölder and Young's inequalities, and recalling that the bilinear form $B_{0}$ is bounded from $H^{2}(\Omega) \times L^{2}(\Omega)$ into $\left(H^{1}(\Omega)\right)^{*}$, we have

$$
\begin{aligned}
\left|I_{1}\right| & \leq C_{8}\left\|J_{\epsilon} v_{\epsilon}\right\|_{H^{2}}\left|v_{1 \epsilon}\right|\left\|u_{\epsilon}\right\| \\
& \leq \delta a_{0}\left(u_{\epsilon}, u_{\epsilon}\right)+\frac{C_{9}}{4 \delta}\left\|J_{\epsilon} v_{\epsilon}\right\|_{H^{2}}^{2}\left|v_{1 \epsilon}\right|^{2} \\
& \leq \delta a_{0}\left(u_{\epsilon}, u_{\epsilon}\right)+\frac{C_{10}}{\delta \epsilon^{2}}\left|v_{1 \epsilon}\right|^{2}\left|v_{\epsilon}\right|^{2},
\end{aligned}
$$

for any $\delta>0$ (a suitable value will be selected later on). Here $C_{9}, C_{10}>0$ depend on $\nu, \beta>0$, but are independent of $\sigma$. Similarly, we also have

$$
\begin{aligned}
\left|I_{2}\right| & \leq C_{11}\left\|J_{\epsilon} v_{2 \epsilon}\right\|\left|v_{\epsilon}\right|\left\|u_{\epsilon}\right\| \\
& \leq \delta a_{0}\left(u_{\epsilon}, u_{\epsilon}\right)+\frac{C_{11}}{\delta \epsilon^{2}}\left|v_{2 \epsilon}\right|^{2}\left|v_{\epsilon}\right|^{2} .
\end{aligned}
$$

Next, on account of the fact that $f_{\epsilon}$ is globally Lipschitz, we find

$$
\begin{aligned}
\left|I_{3}\right| & \leq\left\|\left(f_{\epsilon}\left(\varphi_{1 \epsilon}\right)-f_{\epsilon}\left(\varphi_{2 \epsilon}\right)\right) \nabla\left(J_{\epsilon} \varphi_{1 \epsilon}\right)\right\|_{\left(H^{1}(\Omega)\right)^{*}}\left\|u_{\epsilon}\right\| \\
& \leq \delta a_{0}\left(u_{\epsilon}, u_{\epsilon}\right)+\frac{C_{12}}{\delta \epsilon^{4}}\left\|\varphi_{1 \epsilon}\right\|_{H^{1}}^{2}\left\|\varphi_{\epsilon}\right\|_{H^{1}}^{2}
\end{aligned}
$$

and

$$
\begin{aligned}
\left|I_{5}\right| & \leq\left\|\Delta \varphi_{\epsilon} \nabla\left(J_{\epsilon} \varphi_{1 \epsilon}\right)\right\|_{2}\left|u_{\epsilon}\right| \\
& \leq \delta\left\|\left(\varphi_{\epsilon}, \eta_{\epsilon}\right)\right\|_{V_{\gamma}^{2}}^{2}\left\|\varphi_{1 \epsilon}\right\|_{H^{1}}^{2}+\frac{C_{13}}{\delta \epsilon^{4}}\left|u_{\epsilon}\right|^{2},
\end{aligned}
$$

for any $\delta>0$ and some constant $C_{12}=C_{12}(\epsilon)>0$ which also depends on $c_{f, \epsilon}(\mathrm{cf}$. (5.1)). In the same fashion, for $\sigma \in[0,1]$ we deduce that

$$
\begin{aligned}
\left|I_{4}+I_{6}\right|+\left|I_{-1}+I_{0}\right| & \leq\left(\frac{\delta}{2}\left\|\left(\varphi_{2 \epsilon}, \eta_{2 \epsilon}\right)\right\|_{V_{\gamma}^{2}}^{2}\left\|\varphi_{\epsilon}\right\|_{H^{1}}^{2}+\frac{C_{14}}{\delta \epsilon^{4}}\left|u_{\epsilon}\right|^{2}\right) \\
& +\left(\frac{\delta}{2} \sigma\left\|\partial_{t} \varphi_{2 \epsilon}\right\|_{2}^{2}\left\|\varphi_{\epsilon}\right\|_{H^{1}}^{2}+\frac{C_{14}}{\delta \epsilon^{4}}\left|u_{\epsilon}\right|^{2}\right) \\
& +\left(\delta \sigma\left\|\partial_{t} \varphi_{\epsilon}\right\|_{2}^{2}\left\|\varphi_{1 \epsilon}\right\|_{H^{1}}^{2}+\frac{C_{14}}{\delta \epsilon^{4}}\left|u_{\epsilon}\right|^{2}\right),
\end{aligned}
$$

owing to the fact that $J_{\epsilon} \varphi_{i \epsilon} \in H^{3}(\Omega)$. For the last two terms, owing to the fact that $g_{\epsilon}$ is globally Lipschitz, we have

$$
\begin{aligned}
\left|I_{8}\right| & \leq\left\|\mathcal{L}_{\epsilon}\left(\eta_{2 \epsilon}\right)\right\|_{2, \Gamma}\left\|\nabla_{\tau}\left(K_{\epsilon} \eta_{\epsilon}\right)\right\|_{H^{2}(\Gamma)}\left\|u_{\epsilon \tau}\right\|_{2, \Gamma} \\
& \leq \frac{C_{15}}{\epsilon^{3}}\left\|\left(\varphi_{2 \epsilon}, \eta_{2 \epsilon}\right)\right\|_{V_{\gamma}^{2}}\left\|\eta_{\epsilon}\right\|_{2, \Gamma}\left\|u_{\epsilon \tau}\right\|_{2, \Gamma} \\
& \leq \delta\left\|\left(\varphi_{2 \epsilon}, \eta_{2 \epsilon}\right)\right\|_{V_{\gamma}^{2}}^{2}\left\|\eta_{\epsilon}\right\|_{2, \Gamma}^{2}+\frac{C_{15}}{\delta \epsilon^{6}}\left(\widetilde{\delta}\left|D\left(u_{\epsilon}\right)\right|^{2}+\widetilde{\delta}^{-\alpha}\left|u_{\epsilon}\right|^{2}\right) \\
& \leq \delta\left\|\left(\varphi_{2 \epsilon}, \eta_{2 \epsilon}\right)\right\|_{V_{\gamma}^{2}}^{2}\left\|\eta_{\epsilon}\right\|_{2, \Gamma}^{2}+\delta a_{0}\left(u_{\epsilon}, u_{\epsilon}\right)+\frac{C_{15}}{\delta \epsilon^{6} \widetilde{\delta}^{\alpha}}\left|u_{\epsilon}\right|^{2},
\end{aligned}
$$

by virtue of Lemma 4.3 and the fact that $\left(v_{i \epsilon}, \varphi_{i \epsilon}, \eta_{i \epsilon}\right) \in Z_{T}$. Let us split $I_{7}$ into two additional terms $I_{71}$ and $I_{72}$. Arguing as above, we find

$$
\begin{aligned}
\left|I_{72}\right| & =\left|\left(\left(g_{\epsilon}\left(\eta_{1 \epsilon}\right)-g_{\epsilon}\left(\eta_{2 \epsilon}\right)\right) \nabla_{\tau}\left(K_{\epsilon} \eta_{1 \epsilon}\right), u_{\epsilon \tau}\right)_{\Gamma}\right| \\
& \leq C_{16}\left\|\eta_{\epsilon}\right\|_{2, \Gamma}\left\|K_{\epsilon} \eta_{1 \epsilon}\right\|_{H^{3}(\Gamma)}\left(\widetilde{\delta}\left|D\left(u_{\epsilon}\right)\right|^{2}+\widetilde{\delta}^{-\alpha}\left|u_{\epsilon}\right|^{2}\right)^{1 / 2}
\end{aligned}
$$


and

$$
\begin{aligned}
& \leq \delta\left\|\eta_{\epsilon}\right\|_{2, \Gamma}^{2}\left\|\eta_{1 \epsilon}\right\|_{2, \Gamma}^{2}+\frac{C_{17}}{\delta \epsilon^{6}}\left(\widetilde{\delta}\left|D\left(u_{\epsilon}\right)\right|^{2}+\widetilde{\delta}^{-\alpha}\left|u_{\epsilon}\right|^{2}\right) \\
& \leq \delta\left\|\eta_{\epsilon}\right\|_{2, \Gamma}^{2}\left\|\eta_{1 \epsilon}\right\|_{2, \Gamma}^{2}+\delta a_{0}\left(u_{\epsilon}, u_{\epsilon}\right)+\frac{C_{17}}{\delta \epsilon^{6} \widetilde{\delta}^{\alpha}}\left|u_{\epsilon}\right|^{2}
\end{aligned}
$$

$$
\begin{aligned}
\left|I_{71}\right| & =\left|\left(\widehat{\mathcal{L}}_{\epsilon}\left(\eta_{\epsilon}\right) \nabla_{\tau}\left(K_{\epsilon} \eta_{1 \epsilon}\right), u_{\epsilon \tau}\right)_{\Gamma}\right| \\
& \leq \frac{C_{18}}{\epsilon^{3}}\left\|\left(\varphi_{\epsilon}, \eta_{\epsilon}\right)\right\|_{V_{\gamma}^{2}}\left\|\eta_{1 \epsilon}\right\|_{2, \Gamma}\left(\widetilde{\delta}\left|D\left(u_{\epsilon}\right)\right|^{2}+\widetilde{\delta}^{-\alpha}\left|u_{\epsilon}\right|^{2}\right)^{1 / 2} \\
& \leq \delta\left\|\left(\varphi_{\epsilon}, \eta_{\epsilon}\right)\right\|_{V_{\gamma}^{2}}^{2}\left\|\eta_{1 \epsilon}\right\|_{2, \Gamma}^{2}+\frac{C_{19}}{\delta \epsilon^{6}}\left(\widetilde{\delta}\left|D\left(u_{\epsilon}\right)\right|^{2}+\widetilde{\delta}^{-\alpha}\left|u_{\epsilon}\right|^{2}\right) \\
& \leq \delta\left\|\left(\varphi_{\epsilon}, \eta_{\epsilon}\right)\right\|_{V_{\gamma}^{2}}^{2}\left\|\eta_{1 \epsilon}\right\|_{2, \Gamma}^{2}+\delta a_{0}\left(u_{\epsilon}, u_{\epsilon}\right)+\frac{C_{19}}{\delta \epsilon^{6} \widetilde{\delta}^{\alpha}}\left|u_{\epsilon}\right|^{2} .
\end{aligned}
$$

Here $C_{16}, \ldots, C_{18}>0$ also depend on $c_{g, \epsilon}$ (see (5.1)) and we have chosen $\widetilde{\delta} \in(0,1)$ such that

$$
\widetilde{\delta}=\min \left\{\frac{1}{2}, \frac{\delta^{2} \epsilon^{6}}{C_{17}}, \frac{\delta^{2} \epsilon^{6}}{C_{19}}, \frac{\delta^{2} \epsilon^{6}}{C_{15}}\right\} .
$$

Inserting all the foregoing estimates (5.27)-(5.33) into the right-hand side of (5.26) and using (5.15), we obtain

$$
\begin{aligned}
& \frac{1}{2} \underset{t \in(0, T)}{e s s \sup }\left|u_{\epsilon}(t)\right|^{2}+\int_{0}^{T}(1-6 \delta) a_{0}\left(u_{\epsilon}(t), u_{\epsilon}(t)\right)+\left\|\partial_{t} u_{\epsilon}(t)\right\|_{\left(\mathbb{H}^{1}\right)^{*}}^{2} d t \\
& \leq 2 \delta M_{2}\left(\left\|\left(\varphi_{\epsilon}, \eta_{\epsilon}\right)\right\|_{L^{2}\left(0, T ; V_{\gamma}^{2}\right)}^{2}+\left\|\varphi_{\epsilon}\right\|_{L^{\infty}\left(0, T ; H^{1}\right)}^{2}+\sigma\left\|\partial_{t} \varphi_{\epsilon}\right\|_{L^{2}\left(0, T ; L^{2}\right)}^{2}\right) \\
& +\frac{\left(C_{10}+C_{11}\right) M_{1} T}{\delta \epsilon^{2}}\left\|v_{\epsilon}\right\|_{L^{\infty}(0, T ; \mathbb{H})}^{2}+\frac{C_{12} M_{2} T}{\delta \epsilon^{4}}\left\|\varphi_{\epsilon}\right\|_{L^{\infty}\left(0, T ; H^{1}(\Omega)\right)}^{2} \\
& +\left(\frac{C_{13}+3 C_{14}}{\delta \epsilon^{4}}+\frac{C_{15}+C_{17}+C_{19}}{\delta \epsilon^{6} \widetilde{\delta}^{\alpha}}\right) T\left|u_{\epsilon}\right|_{L^{\infty}(0, T ; \mathbb{H})}^{2} .
\end{aligned}
$$

Let us choose

$$
\delta=\min \left\{\frac{1}{12}, \frac{1}{24 M_{2}}\right\}<1
$$

and pick a time $T=T_{\epsilon}>0$ such that

$$
\left\{\begin{array}{l}
\left(\frac{C_{13}+3 C_{14}}{\delta \epsilon^{4}}+\frac{C_{15}+C_{17}+C_{19}}{\delta \epsilon^{6} \tilde{\delta}^{\alpha}}\right) T \leq \frac{1}{4} \\
\max \left\{\frac{\left(C_{10}+C_{11}\right) M_{1}}{\delta \epsilon^{2}}, \frac{C_{12} M_{2}}{\delta \epsilon^{4}}\right\} T \leq \frac{1}{12}
\end{array}\right.
$$

With these choices, (5.35) yields the inequality

$$
\left\|u_{1 \epsilon}(t)-u_{2 \epsilon}(t)\right\|_{X_{T}}^{2} \leq \frac{1}{3}\left\|\left(v_{\epsilon}(t), \varphi_{\epsilon}(t), \eta_{\epsilon}(t)\right)\right\|_{Z_{T}}^{2}
$$

for all $t \in\left(0, T_{\epsilon}\right)$.

We now estimate $\left(\phi_{\epsilon}, \psi_{\epsilon}\right)$ in the space $Y_{T}$. Pairing the first and second equations of (5.25) with $A_{N}^{-1}\left(\partial_{t} \phi_{\epsilon}\right)$ and $\partial_{t} \psi_{\epsilon}$ with the scalar products of $L^{2}(\Omega)$ and $L^{2}(\Gamma)$, respectively, and then pairing in $L^{2}(\Omega)$ the first equation of $(5.25)$ with $\bar{\mu}_{\epsilon}-\left\langle\bar{\mu}_{\epsilon}\right\rangle$, after elementary computations we find

$$
\begin{aligned}
& \frac{1}{2}\left\|\left(\phi_{\epsilon}, \psi_{\epsilon}\right)\right\|_{L^{\infty}\left(0, T ; V_{\gamma}^{1}\right)}^{2}+\sigma\left\|\partial_{t} \phi_{\epsilon}\right\|_{L^{2}\left(0, T ; L^{2}(\Omega)\right)}^{2} \\
& +\left\|\left(\phi_{\epsilon}, \psi_{\epsilon}\right)\right\|_{H^{1}\left(0, T ;\left(H^{1}(\Omega)\right)^{*} \times L^{2}(\Gamma)\right)}^{2}+\left\|\nabla \bar{\mu}_{\epsilon}\right\|_{L^{2}\left(0, T ; L^{2}(\Omega)^{N}\right)}^{2} \\
& \leq 2 \sum_{i=9}^{16} \int_{0}^{T} I_{i}(t) d t,
\end{aligned}
$$

where we have set

$$
\begin{cases}I_{9}:=-b_{1}\left(v_{\epsilon}, J_{\epsilon} \varphi_{1 \epsilon}, A_{N}^{-1}\left(\partial_{t} \phi_{\epsilon}\right)\right), & I_{10}:=-b_{1}\left(v_{2 \epsilon}, J_{\epsilon} \varphi_{\epsilon}, A_{N}^{-1}\left(\partial_{t} \phi_{\epsilon}\right)\right), \\ I_{11}:=\left(f_{\epsilon}\left(\varphi_{1 \epsilon}\right)-f_{\epsilon}\left(\varphi_{2 \epsilon}\right), \partial_{t} \phi_{\epsilon}\right), & I_{12}:=-\left(B_{\Gamma}\left(v_{\epsilon}, K_{\epsilon} \eta_{1 \epsilon}\right), \partial_{t} \psi_{\epsilon}\right)_{\Gamma}, \\ I_{13}:=-\left(B_{\Gamma}\left(v_{2 \epsilon}, K_{\epsilon} \eta_{\epsilon}\right), \partial_{t} \psi_{\epsilon}\right)_{\Gamma}, & I_{14}:=-\left(g_{\epsilon}\left(\eta_{1 \epsilon}\right)-g_{\epsilon}\left(\eta_{2 \epsilon}\right), \partial_{t} \psi_{\epsilon}\right)_{\Gamma}\end{cases}
$$


and

$$
I_{15}:=-\left(B_{1}\left(v_{\epsilon}, J_{\epsilon} \varphi_{1 \epsilon}\right), \bar{\mu}_{\epsilon}-\left\langle\bar{\mu}_{\epsilon}\right\rangle\right), \quad I_{16}:=-\left(B_{1}\left(v_{2 \epsilon}, J_{\epsilon} \varphi_{\epsilon}\right), \bar{\mu}_{\epsilon}-\left\langle\bar{\mu}_{\epsilon}\right\rangle\right) .
$$

Next, recalling the definitions of $\bar{\mu}_{\epsilon}$ and $\widehat{\mathcal{L}}_{\epsilon}\left(\psi_{\epsilon}\right)$, we obtain, since $\left\langle\partial_{t} \phi_{\epsilon}(t)\right\rangle=0$,

$$
\left\langle\bar{\mu}_{\epsilon}\right\rangle=\left\langle f_{\epsilon}\left(\varphi_{1 \epsilon}\right)-f_{\epsilon}\left(\varphi_{2 \epsilon}\right)\right\rangle-|\Gamma|\left\langle\widehat{\mathcal{L}}_{\epsilon}\left(\psi_{\epsilon}\right)\right\rangle_{\Gamma}+|\Gamma| \zeta\left\langle\psi_{\epsilon}\right\rangle_{\Gamma}
$$

so that (cf. the second equation of (5.25))

$$
\begin{aligned}
\left\langle\bar{\mu}_{\epsilon}\right\rangle^{2} & \leq C_{\#}\left(\left\|\partial_{t} \psi_{\epsilon}\right\|_{\Gamma}^{2}+\left\|f_{\epsilon}\left(\varphi_{1 \epsilon}\right)-f_{\epsilon}\left(\varphi_{2 \epsilon}\right)\right\|_{2}^{2}+\left\|g_{\epsilon}\left(\eta_{1 \epsilon}\right)-g_{\epsilon}\left(\eta_{2 \epsilon}\right)\right\|_{\Gamma}^{2}\right) \\
& +C_{\#}\left(\left\|B_{\Gamma}\left(v_{2 \epsilon}, K_{\epsilon} \eta_{\epsilon}\right)\right\|_{\Gamma}^{2}+\left\|B_{\Gamma}\left(v_{\epsilon}, K_{\epsilon} \eta_{1 \epsilon}\right)\right\|_{\Gamma}^{2}\right),
\end{aligned}
$$

for some $C_{\#}>0$ which depends on $|\Gamma|$ and $|\Omega|$, but is independent of $\sigma$. Furthermore, from this estimate together with (5.39) and the elliptic regularity result of Lemma 4.6, we can infer the existence of a further constant $C_{*}>0$, depending on $C_{\#}$, such that

$$
\begin{aligned}
& \frac{1}{2}\left\|\left(\phi_{\epsilon}, \psi_{\epsilon}\right)\right\|_{L^{\infty}\left(0, T ; V_{\gamma}^{1}\right)}^{2}+\left\|\left(\phi_{\epsilon}, \psi_{\epsilon}\right)\right\|_{H^{1}\left(0, T ;\left(H^{1}(\Omega)\right)^{*} \times L^{2}(\Gamma)\right)}^{2}+\sigma\left\|\partial_{t} \phi_{\epsilon}\right\|_{L^{2}\left(0, T ; L^{2}(\Omega)\right)}^{2} \\
& +\left\|\bar{\mu}_{\epsilon}\right\|_{L^{2}\left(0, T ; H^{1}(\Omega)\right)}^{2}+\left\|\left(\phi_{\epsilon}, \psi_{\epsilon}\right)\right\|_{L^{2}\left(0, T ; V_{\gamma}^{2}\right)}^{2} \\
& \leq C_{*} \sum_{i=9}^{19} \int_{0}^{T}\left|I_{i}(t)\right| d t
\end{aligned}
$$

where

$$
\begin{aligned}
& I_{17}:=\left\|B_{\Gamma}\left(v_{2 \epsilon}, K_{\epsilon} \eta_{\epsilon}\right)\right\|_{\Gamma}^{2}, \quad I_{18}:=\left\|B_{\Gamma}\left(v_{\epsilon}, K_{\epsilon} \eta_{1 \epsilon}\right)\right\|_{\Gamma}^{2}, \\
& I_{19}:=\left\|f_{\epsilon}\left(\varphi_{1 \epsilon}\right)-f_{\epsilon}\left(\varphi_{2 \epsilon}\right)\right\|_{2}^{2}+\left\|g_{\epsilon}\left(\eta_{1 \epsilon}\right)-g_{\epsilon}\left(\eta_{2 \epsilon}\right)\right\|_{\Gamma}^{2} .
\end{aligned}
$$

To estimate $I_{9}$ and $I_{10}$, we observe that each one of the terms $\left(v_{\epsilon}\right)_{i} \partial_{i}\left(J_{\epsilon} \varphi_{1 \epsilon}\right),\left(v_{2 \epsilon}\right)_{i} \partial_{i}\left(J_{\epsilon} \varphi_{\epsilon}\right)$ $(1 \leq i \leq \mathrm{N})$ is a product of functions in $L^{2}(\Omega)$ and $H^{2}(\Omega) \hookrightarrow C^{0, \vartheta}(\bar{\Omega})$, respectively. Therefore they are bounded in $\left(H^{1}(\Omega)\right)^{*}$. Thus, we deduce that

$$
\begin{aligned}
\left|I_{9}\right| & \leq\left\|B_{1}\left(v_{\epsilon}, J_{\epsilon} \varphi_{1 \epsilon}\right)\right\|_{\left(H^{1}(\Omega)\right)^{*}}\left\|\partial_{t} \phi_{\epsilon}\right\|_{\left(H^{1}(\Omega)\right)^{*}} \\
& \leq \varpi\left\|\partial_{t} \phi_{\epsilon}\right\|_{\left(H^{1}(\Omega)\right)^{*}}^{2}+\frac{C_{20}}{\varpi \epsilon^{4}}\left|v_{\epsilon}\right|^{2}\left\|\varphi_{1 \epsilon}\right\|_{H^{1}}^{2}
\end{aligned}
$$

and

$$
\begin{aligned}
\left|I_{10}\right| & \leq\left\|B_{1}\left(v_{2 \epsilon}, J_{\epsilon} \varphi_{\epsilon}\right)\right\|_{\left(H^{1}(\Omega)\right)^{*}}\left\|\partial_{t} \phi_{\epsilon}\right\|_{\left(H^{1}(\Omega)\right)^{*}} \\
& \leq \varpi\left\|\partial_{t} \phi_{\epsilon}\right\|_{\left(H^{1}(\Omega)\right)^{*}}^{2}+\frac{C_{21}}{\varpi \epsilon^{4}}\left|v_{2 \epsilon}\right|^{2}\left\|\varphi_{\epsilon}\right\|_{H^{1}}^{2},
\end{aligned}
$$

for any $\varpi>0$ to be chosen later on. Bounds on $I_{11}$ and $I_{14}$ can be obtained quite easily on account of the fact that $f_{\epsilon}$ and $g_{\epsilon}$ satisfy (5.1). In particular, we have

$$
\left|I_{11}+I_{14}\right| \leq \varpi\left(\left\|\partial_{t} \phi_{\epsilon}\right\|_{\left(H^{1}(\Omega)\right)^{*}}^{2}+\left\|\partial_{t} \psi_{\epsilon}\right\|_{2, \Gamma}^{2}\right)+\frac{C_{22}}{\varpi}\left\|\varphi_{\epsilon}\right\|_{H^{1}}^{2}
$$

for some constant $C_{22}=C_{22}(\epsilon)>0$ depending on $c_{f, \epsilon}, c_{g, \epsilon}$ (cf. (5.1)), but which is independent of $\sigma$. We will exploit Lemma 4.3 once again to bound the energy terms $I_{12}$ and $I_{13}$. By Hölder and Young inequalities, we get

$$
\begin{aligned}
\left|I_{12}\right| & \leq\left\|\partial_{t} \psi_{\epsilon}\right\|_{2, \Gamma}\left\|v_{\epsilon \tau}\right\|_{2, \Gamma}\left\|K_{\epsilon} \eta_{1 \epsilon}\right\|_{H^{3}(\Gamma)} \\
& \leq \frac{C_{23}}{\epsilon^{3}}\left\|\eta_{1 \epsilon}\right\|_{2, \Gamma}\left(\varepsilon\left|D\left(v_{\epsilon}\right)\right|^{2}+\varepsilon^{-\alpha}\left|v_{\epsilon}\right|^{2}\right)^{1 / 2}\left\|\partial_{t} \psi_{\epsilon}\right\|_{2, \Gamma} \\
& \leq \varpi\left\|\partial_{t} \psi_{\epsilon}\right\|_{2, \Gamma}^{2}+\frac{C_{24}}{\varpi \epsilon^{6}}\left\|\eta_{1 \epsilon}\right\|_{2, \Gamma}^{2}\left(\varepsilon\left|D\left(v_{\epsilon}\right)\right|^{2}+\varepsilon^{-\alpha}\left|v_{\epsilon}\right|^{2}\right) \\
& \leq \varpi\left\|\partial_{t} \psi_{\epsilon}\right\|_{2, \Gamma}^{2}+\frac{1}{12 C_{*} M_{2}}\left\|\eta_{1 \epsilon}\right\|_{2, \Gamma}^{2}\left\|v_{\epsilon}\right\|^{2}+\frac{C_{24}}{\varpi \epsilon^{6} \varepsilon^{\alpha}}\left\|\eta_{1 \epsilon}\right\|_{2, \Gamma}^{2}\left|v_{\epsilon}\right|^{2},
\end{aligned}
$$

taking

$$
\varepsilon=\min \left\{\frac{1}{12}, \frac{\varpi \epsilon^{6}}{12 M_{2} C_{24} C_{*}}, \frac{\varpi \epsilon^{6}}{12 M_{1} C_{25} C_{*}}, \frac{\epsilon^{3}}{12 C_{*} C_{28} M_{2}}, \frac{\epsilon^{3}}{12 C_{*} C_{29} M_{1}}\right\}<1
$$


Arguing as above, we also deduce that

$$
\begin{aligned}
\left|I_{13}\right| & \leq\left\|\partial_{t} \psi_{\epsilon}\right\|_{2, \Gamma}\left\|v_{2 \epsilon \tau}\right\|_{2, \Gamma}\left\|K_{\epsilon} \eta_{\epsilon}\right\|_{H^{3}(\Gamma)} \\
& \leq \varpi\left\|\partial_{t} \psi_{\epsilon}\right\|_{2, \Gamma}^{2}+\frac{C_{25}}{\varpi \epsilon^{6}}\left\|\eta_{\epsilon}\right\|_{2, \Gamma}^{2}\left(\varepsilon\left|D\left(v_{2 \epsilon}\right)\right|^{2}+\varepsilon^{-\alpha}\left|v_{2 \epsilon}\right|^{2}\right) \\
& \leq \varpi\left\|\partial_{t} \psi_{\epsilon}\right\|_{2, \Gamma}^{2}+\frac{1}{12 C_{*} M_{1}}\left\|\eta_{\epsilon}\right\|_{2, \Gamma}^{2}\left\|v_{2 \epsilon}\right\|^{2}+\frac{C_{25}}{\varpi \epsilon^{6} \varepsilon^{\alpha}}\left\|\eta_{\epsilon}\right\|_{2, \Gamma}^{2}\left|v_{2 \epsilon}\right|^{2} .
\end{aligned}
$$

In the same fashion as in (5.42)-(5.43), by virtue of Poincare's inequality we obtain

$$
\left|I_{15}+I_{16}\right| \leq \varpi\left\|\nabla \bar{\mu}_{\epsilon}\right\|_{2}^{2}+\frac{C_{26}}{\varpi \epsilon^{4}}\left(\left|v_{2 \epsilon}\right|^{2}\left\|\varphi_{\epsilon}\right\|_{H^{1}}^{2}+\left|v_{\epsilon}\right|^{2}\left\|\varphi_{1 \epsilon}\right\|_{H^{1}}^{2}\right) .
$$

The term $I_{17}$ is straightforward. Indeed, owing to (5.1), we have

$$
\left|I_{17}\right| \leq C_{27}\left(\left\|\varphi_{\epsilon}\right\|_{2}^{2}+\left\|\eta_{\epsilon}\right\|_{2, \Gamma}^{2}\right) .
$$

The remaining ones can be estimated by employing the argument used in (5.45) and (5.46). We get

$$
\begin{aligned}
\left|I_{18}\right| & \leq \frac{C_{28}}{\epsilon^{3}}\left\|v_{2 \epsilon \tau}\right\|_{2, \Gamma}^{2}\left\|\eta_{\epsilon}\right\|_{2, \Gamma}^{2} \\
& \leq \frac{C_{28}}{\epsilon^{3}}\left(\varepsilon\left|D\left(v_{2 \epsilon}\right)\right|^{2}+\varepsilon^{-\alpha}\left|v_{2 \epsilon}\right|^{2}\right)\left\|\eta_{\epsilon}\right\|_{2, \Gamma}^{2} \\
& \leq \frac{1}{12 C_{*} M_{1}}\left\|\eta_{\epsilon}\right\|_{2, \Gamma}^{2}\left\|v_{2 \epsilon}\right\|^{2}+\frac{C_{28}}{\epsilon^{3} \varepsilon^{\alpha}}\left\|\eta_{\epsilon}\right\|_{2, \Gamma}^{2}\left|v_{2 \epsilon}\right|^{2}
\end{aligned}
$$

and, similarly, we find

$$
\left|I_{19}\right| \leq \frac{1}{12 C_{*} M_{2}}\left\|\eta_{1 \epsilon}\right\|_{2, \Gamma}^{2}\left\|v_{\epsilon}\right\|^{2}+\frac{C_{29}}{\epsilon^{3} \varepsilon^{\alpha}}\left\|\eta_{1 \epsilon}\right\|_{2, \Gamma}^{2}\left|v_{\epsilon}\right|^{2}
$$

On account of estimates (5.42)-(5.50), it follows from (5.41) that (cf. also (5.15))

$$
\begin{aligned}
& \frac{1}{2}\left\|\left(\phi_{\epsilon}, \psi_{\epsilon}\right)\right\|_{L^{\infty}\left(0, T ; V_{\gamma}^{1}\right)}^{2}+\left\|\left(\phi_{\epsilon}, \psi_{\epsilon}\right)\right\|_{H^{1}\left(0, T ;\left(H^{1}(\Omega)\right)^{*} \times L^{2}(\Gamma)\right)}^{2}+\sigma\left\|\partial_{t} \phi_{\epsilon}\right\|_{L^{2}\left(0, T ; L^{2}(\Omega)\right)}^{2} \\
& +\left\|\left(\phi_{\epsilon}, \psi_{\epsilon}\right)\right\|_{L^{2}\left(0, T ; V_{\gamma}^{2}\right)}^{2}+\left\|\nabla \bar{\mu}_{\epsilon}\right\|_{L^{2}\left(0, T ; L^{2}(\Omega)^{N}\right)}^{2} \\
& \leq 3 \varpi C_{*}\left(\left\|\partial_{t} \phi_{\epsilon}\right\|_{L^{2}\left(0, T ;\left(H^{1}(\Omega)\right)^{*}\right)}^{2}+\left\|\partial_{t} \psi_{\epsilon}\right\|_{L^{2}\left(0, T ; L^{2}(\Gamma)\right)}^{2}\right)+\varpi C_{*}\left\|\nabla \bar{\mu}_{\epsilon}\right\|_{L^{2}\left(0, T ; L^{2}(\Omega)^{N}\right)}^{2} \\
& +C_{*}\left(\frac{C_{21} M_{1}}{\varpi \epsilon^{4}}+\frac{C_{22}}{\varpi}\right) T\left\|\varphi_{\epsilon}\right\|_{L^{\infty}\left(0, T ; H^{1}\right)}^{2}+\frac{C_{*} C_{20} M_{2} T}{\varpi \epsilon^{4}}\left\|v_{\epsilon}\right\|_{L^{\infty}(0, T ; \mathbb{H})}^{2} \\
& +\left(\frac{1}{6}+\frac{C_{*} C_{24} M_{2} T}{\varpi \epsilon^{6} \varepsilon^{\alpha}}+\frac{C_{26} C_{*} M_{2} T}{\varpi \epsilon^{4}}+\frac{C_{29} C_{*} M_{2} T}{\epsilon^{3} \varepsilon^{\alpha}}\right)\left\|v_{\epsilon}\right\|_{L^{\infty}(0, T ; \mathbb{H})}^{2} \\
& +\left(\frac{1}{6}+\frac{C_{*} C_{24} M_{1} T}{\varpi \epsilon^{6} \varepsilon^{\alpha}}+\frac{C_{26} C_{*} M_{1} T}{\varpi \epsilon^{4}}+\frac{C_{28} C_{*} M_{1} T}{\epsilon^{3} \varepsilon^{\alpha}}\right)\left\|\varphi_{\epsilon}\right\|_{L^{\infty}\left(0, T ; H^{1}(\Omega)\right)}^{2} .
\end{aligned}
$$

We can now choose any $0<\varpi \leq 1 /\left(6 C_{*}\right)$ together with a time $T=T_{\epsilon}>0$ (which is independent of $\sigma$ ) such that

$$
\left\{\begin{array}{l}
\max \left\{\frac{C_{20} M_{2}}{\varpi \epsilon^{4}}, \frac{C_{21} M_{1}}{\varpi \epsilon^{4}}+\frac{C_{22}}{\varpi}\right\} T \leq \frac{1}{12 C_{*}} \\
\left(\frac{C_{24} M_{2}}{\varpi \epsilon^{6} \varepsilon^{\alpha}}+\frac{C_{26} M_{2}}{\varpi \epsilon^{4}}+\frac{C_{29} M_{2}}{\epsilon^{3} \varepsilon^{\alpha}}\right) T \leq \frac{1}{12 C_{*}} \\
\left(\frac{C_{24} M_{1}}{\varpi \epsilon^{6} \varepsilon^{\alpha}}+\frac{C_{26} M_{1} T}{\varpi \epsilon^{4}}+\frac{C_{28} M_{1}}{\epsilon^{3} \varepsilon^{\alpha}}\right) T \leq \frac{1}{12 C_{*}}
\end{array} .\right.
$$

This choice allows us to deduce from (5.51) that

$$
\left\|\left(\phi_{1 \epsilon}(t)-\phi_{2 \epsilon}(t), \psi_{1 \epsilon}(t)-\psi_{2 \epsilon}(t)\right)\right\|_{Y_{T}}^{2} \leq \frac{1}{3}\left\|\left(v_{\epsilon}(t), \varphi_{\epsilon}(t), \eta_{\epsilon}(t)\right)\right\|_{Z_{T}}^{2}
$$

for all $t \in\left(0, T_{\epsilon}\right)$. Hence, on account of (5.38) and (5.53), we can say that $S$ is a contraction from $Z_{T}$ into itself, provided that $T=T_{\epsilon}>0$ also satisfies (5.37), (5.52) and is possibly a number smaller than the one which fulfills (5.19) and (5.23). Therefore, owing to the contraction mapping principle, we conclude that problem $\mathbf{P}_{\epsilon, \sigma}$ has a unique local solution $\left(u_{\epsilon, \sigma}, \phi_{\epsilon, \sigma}, \psi_{\epsilon, \sigma}\right) \in Z_{T}$, for each $\epsilon, \sigma \in(0,1]$.

Henceforth, we have proved the following existence result on weak solutions for problem $\mathbf{P}_{\epsilon, \sigma}$. 
Theorem 5.1. For each $\epsilon, \sigma>0$ and initial condition $\left(u_{0}, \phi_{0}, \psi_{0}\right) \in \mathbb{H} \times V_{\gamma}^{1}$, problem $\boldsymbol{P}_{\epsilon, \sigma}$ has a unique (local in time) solution

$$
\Upsilon_{\epsilon, \sigma}:=\left(u_{\epsilon, \sigma}, \phi_{\epsilon, \sigma}, \psi_{\epsilon, \sigma}\right) \in L^{\infty}\left(0, T_{\epsilon} ; \mathbb{H} \times V_{\gamma}^{1}\right),
$$

for some $T_{\epsilon}>0$. Furthermore, this solution possesses the following regularity

$$
\left\{\begin{array}{l}
u_{\epsilon, \sigma} \in H^{1}\left(0, T_{\epsilon} ;\left(\mathbb{H}^{1}\right)^{*}\right) \cap L^{2}\left(0, T_{\epsilon} ; \mathbb{H}\right), \\
\left(\phi_{\epsilon, \sigma}, \psi_{\epsilon, \sigma}\right) \in H^{1}\left(0, T_{\epsilon} ; L^{2}(\Omega) \times L^{2}(\Gamma)\right) \cap L^{2}\left(0, T_{\epsilon} ; V_{\gamma}^{2}\right)
\end{array}\right.
$$

and solves $\boldsymbol{P}_{\epsilon, \sigma}$ in the sense of (5.3)-(5.8). In particular, $\Upsilon_{\epsilon, \sigma}(t)$ is almost everywhere equal to an absolutely continuous function from $\left(0, T_{\epsilon}\right)$ into $\mathbb{H} \times V_{\gamma}^{1}$.

Proof. It remains to show the last part of the theorem, but this follows easily from the regularity stated in (5.54) and the straightforward relations

$$
\begin{aligned}
& \frac{d}{d t}\left|u_{\epsilon, \sigma}(t)\right|^{2}=2\left\langle A_{0}^{-1 / 2} \partial_{t} u_{\epsilon, \sigma}(t), A_{0}^{1 / 2} u_{\epsilon, \sigma}(t)\right\rangle, \\
& \frac{d}{d t}\left\|\psi_{\epsilon, \sigma}(t)\right\|_{H^{1}(\Gamma)}^{2}=2\left(\partial_{t} \psi_{\epsilon, \sigma}(t), A_{\tau} \psi_{\epsilon, \sigma}(t)\right)_{\Gamma}, \\
& \frac{d}{d t}\left\|\phi_{\epsilon, \sigma}(t)\right\|_{H^{1}}^{2}=2\left(\partial_{t} \phi_{\epsilon, \sigma}(t),-\Delta \phi_{\epsilon, \sigma}(t)\right)+2\left(\partial_{t} \psi_{\epsilon, \sigma}(t), \partial_{n} \phi_{\epsilon, \sigma}(t)\right)_{\Gamma}
\end{aligned}
$$

which hold in the distributional sense over $\left(0, T_{\epsilon}\right)$.

Remark 5.2. Owing to Theorem 5.1, note that the regularized energy

$$
E_{\epsilon, \sigma}(t):=\frac{1}{2}\left\|\left(u_{\epsilon, \sigma}(t), \phi_{\epsilon, \sigma}(t), \psi_{\epsilon, \sigma}(t)\right)\right\|_{\mathbb{Y}_{\gamma}}^{2}+\int_{\Omega} F_{\epsilon}\left(\phi_{\epsilon, \sigma}(t)\right) d x+\int_{\Gamma} G_{\epsilon}\left(\psi_{\epsilon, \sigma}(t)\right) d S
$$

is almost everywhere equal to an absolutely continuous function on $\left(0, T_{\epsilon}\right)$, since $F_{\epsilon}, G_{\epsilon} \in C^{1}(\mathbb{R})$.

Step 3. The original problem. The final step is to show that a subsequence of weak solutions to $\mathbf{P}_{\epsilon, \sigma}$ converges to a solution to the original system (1.1)-(1.10). To do this, we need estimates that are independent of $\epsilon, \sigma \rightarrow 0$. At the same time, such estimates will allow us to conclude that any regularized solution is also globally well defined over $(0, T)$, for any $T>0$.

We start by first noticing that, for almost any $t \in\left(0, T_{\epsilon}\right)$, the test functions $\varphi=\mu_{\epsilon, \sigma}(t) \in$ $H^{1}(\Omega), \eta=\mathcal{L}\left(\psi_{\epsilon}(t)\right) \in L^{2}(\Gamma)$ and $v=u_{\epsilon}(t) \in \mathbb{H}^{1}$ are admissible in (2.7)-(2.8) and in (2.6), respectively, on account of the regularity of $\Upsilon_{\epsilon, \sigma}$. On the other hand, we can now test the first and second equations of (5.12) and (5.13) in $L^{2}(\Omega)$, and $L^{2}(\Gamma)$, with $\partial_{t} \phi_{\epsilon, \sigma}(t) \in L^{2}(\Omega)$ and $\partial_{t} \psi_{\epsilon, \sigma}(t) \in L^{2}(\Gamma)$, respectively. Adding the resulting identities (see (3.4)-(3.5)), we deduce as in (3.6) (with the exception of the additional term involving $\sigma>0$ ) the following energy inequality

$$
\begin{aligned}
& \frac{d}{d t} E_{\epsilon, \sigma}(t)+C_{\delta}\left\|u_{\epsilon, \sigma}(t)\right\|^{2}+\left\|\nabla \mu_{\epsilon, \sigma}(t)\right\|_{2}^{2} \\
& \quad+\left\|\mathcal{L}\left(\psi_{\epsilon, \sigma}(t)\right)\right\|_{2, \Gamma}^{2}+\sigma\left\|\partial_{t} \phi_{\epsilon, \sigma}(t)\right\|_{2}^{2} \\
& \quad \leq(4 \delta)^{-1}\|h(t)\|_{\left(H^{1}(\Omega)\right)^{*}}^{2},
\end{aligned}
$$

for some $C_{\delta}>0$ independent of $\epsilon, \sigma>0$. Note that, owing to Theorem 5.1 (cf. also Remark 5.2), we have $E_{\epsilon, \sigma} \in A C\left(0, T_{\epsilon}\right)$. On the other hand, we have $E_{\epsilon, \sigma}(0) \leq C$ uniformly in $\epsilon, \sigma>0$, owing to $(5.2)$, provided that $F\left(\phi_{0}\right) \in L^{1}(\Omega)$ and $G\left(\psi_{0}\right) \in L^{1}(\Gamma)$. This is ensured by $\left(\phi_{0}, \psi_{0}\right) \in V_{\gamma}^{1}$ (see $(3.1),(3.2))$. Also, by virtue of (3.1)-(3.2), we notice that

$$
E_{\epsilon, \sigma}(t) \geq \frac{1}{2}\left\|\left(u_{\epsilon, \sigma}(t), \phi_{\epsilon, \sigma}(t), \psi_{\epsilon, \sigma}(t)\right)\right\|_{\mathbb{Y}_{\gamma}}^{2}-C_{F, G},
$$

for some $C_{F, G}>0$, independent of $\epsilon, \sigma>0$. Therefore, the local solution can be extended to $(0, T)$, for any given $T>0$. Integrating (5.55) over $(0, T)$, for any $T>0$, we obtain, on account of assumptions (a)-(b) and (5.2),

$$
\left\{\begin{array}{l}
\left(u_{\epsilon, \sigma}, \phi_{\epsilon, \sigma}, \psi_{\epsilon, \sigma}\right) \in L^{\infty}\left(0, T ; \mathbb{H} \times V_{\gamma}^{1}\right) \\
u_{\epsilon, \sigma} \in L^{2}\left(0, T ; \mathbb{H}^{1}\right) \\
\nabla \mu_{\epsilon, \sigma} \in L^{2}\left(0, T ; L^{2}(\Omega)^{N}\right) \\
\mathcal{L}\left(\psi_{\epsilon, \sigma}\right) \in L^{2}\left(0, T ; L^{2}(\Gamma)\right)
\end{array}\right.
$$

uniformly with respect to $\epsilon, \sigma>0$. In addition, we also have

$$
\sigma^{1 / 2} \partial_{t} \phi_{\epsilon, \sigma} \in L^{2}\left(0, T ; L^{2}(\Omega)\right) \text {. }
$$


Next, integrating (5.6) and (5.7) over $\Omega$ and $\Gamma$, respectively, we further see that $\left\langle\mu_{\epsilon, \sigma}\right\rangle \in L^{2}(0, T)$, uniformly in $\epsilon, \sigma>0$ since $\left\langle\partial_{t} \phi_{\epsilon, \sigma}(t)\right\rangle=0$, almost everywhere on $(0, T)$, and by virtue of the last of (5.56). Then

$$
\mu_{\epsilon, \sigma} \text { is uniformly bounded in } L^{2}\left(0, T ; H^{1}(\Omega)\right) \text {. }
$$

Furthermore, since $\left(\phi_{\epsilon, \sigma}, \psi_{\epsilon, \sigma}\right)$ is also a weak solution to the elliptic boundary value problem

$$
\begin{aligned}
-\Delta \phi_{\epsilon, \sigma} & =H_{1 \epsilon, \sigma}, \quad \text { a.e. in } \Omega \times(0, T), \\
\gamma A_{\tau} \psi_{\epsilon, \sigma}+\partial_{n} \phi_{\epsilon, \sigma}+\zeta \psi_{\epsilon, \sigma} & =H_{2 \epsilon, \sigma}, \quad \text { a.e on } \Gamma \times(0, T),
\end{aligned}
$$

with

$$
\begin{aligned}
& H_{1 \epsilon, \sigma}:=\mu_{\epsilon, \sigma}-\sigma \partial_{t} \phi_{\epsilon, \sigma}-f_{\epsilon}\left(\phi_{\epsilon, \sigma}\right) \in L^{2}\left(0, T ; L^{2}(\Omega)\right), \\
& H_{2 \epsilon, \sigma}:=\mathcal{L}\left(\psi_{\epsilon, \sigma}\right)-g_{\epsilon}\left(\psi_{\epsilon, \sigma}\right) \in L^{2}\left(0, T ; L^{2}(\Gamma)\right)
\end{aligned}
$$

by Lemma 4.6, together with the bound (5.57) for $\sigma \in(0,1]$, we get

$$
\left(\phi_{\epsilon, \sigma}, \psi_{\epsilon, \sigma}\right) \text { is uniformly bounded in } L^{2}\left(0, T ; V_{\gamma}^{2}\right) \text {. }
$$

In order to control the nonlinear terms associated with the forms $B_{0}, B_{1}, B_{\Gamma}$ and functions $f_{\epsilon}, g_{\epsilon}$, we can rely on Lemma 4.7 to show the following uniform bounds with respect to $\epsilon, \sigma>0$. As usual,

$$
B_{0}\left(J_{\epsilon} u_{\epsilon, \sigma}, u_{\epsilon, \sigma}\right) \text { is uniformly bounded in } L^{p}\left(0, T ;\left(\mathbb{H}^{1}\right)^{*}\right) \text {, }
$$

with $p=2$ if $N=2$ and $p=4 / 3$ if $N=3$, owing to Lemma 4.1, (iv). Moreover, we immediately get that

$$
\mu_{\epsilon, \sigma} \nabla J_{\epsilon} \phi_{\epsilon, \sigma} \text { is uniformly bounded in } L^{2}\left(0, T ;\left(\mathbb{H}^{1}\right)^{*}\right)
$$

and

$$
B_{1}\left(u_{\epsilon, \sigma}, J_{\epsilon} \phi_{\epsilon, \sigma}\right) \text { is uniformly bounded in } L^{2}\left(0, T ;\left(H^{1}(\Omega)\right)^{*}\right) \text {. }
$$

Thus, a comparison in equation (2.7) yields

$$
\partial_{t} \phi_{\epsilon, \sigma} \text { is uniformly bounded in } L^{2}\left(0, T ;\left(H^{1}(\Omega)\right)^{*}\right) \text {. }
$$

In order to provide uniform bounds for the time derivatives $\partial_{t} u_{\epsilon, \sigma}$ and $\partial_{t} \psi_{\epsilon, \sigma}$, it suffices to derive uniform bounds on the boundary terms in (2.6) and (2.8), respectively.

Case $N=3$ : Let now $\eta \in L^{4}\left(0, T ; L^{2}(\Gamma)\right)$, such that $\|\eta\|_{L^{4}\left(0, T ; L^{2}(\Gamma)\right)} \leq 1$, and recall that $\left(u_{\epsilon, \sigma}, \psi_{\epsilon, \sigma}\right)$ satisfies (5.56) and (5.59). Thus, by the Sobolev inequality $\left\|\nabla_{\tau} \psi\right\|_{4, \Gamma} \lesssim\|\psi\|_{H^{2}(\Gamma)}^{1 / 2}\left\|\nabla_{\tau} \psi\right\|_{2, \Gamma}^{1 / 2}$, the injections $H^{1}(\Omega) \hookrightarrow H^{1 / 2}(\Gamma) \hookrightarrow L^{4}(\Gamma)$ and Lemma 4.1, (iv), we deduce that

$$
\begin{aligned}
\left|b_{\Gamma}\left(u_{\epsilon, \sigma}, K_{\epsilon} \psi_{\epsilon, \sigma}, \eta\right)\right| & \leq C\left|u_{\tau \epsilon, \sigma}\right|_{4, \Gamma}\left\|\nabla_{\tau} K_{\epsilon} \psi_{\epsilon, \sigma}\right\|_{4, \Gamma}\|\eta\|_{2, \Gamma} \\
& \leq C\left\|u_{\epsilon, \sigma}\right\|\left\|K_{\epsilon} \psi_{\epsilon, \sigma}\right\|_{H^{2}(\Gamma)}^{1 / 2}\left\|K_{\epsilon} \psi_{\epsilon, \sigma}\right\|_{H^{1}(\Gamma)}^{1 / 2}\|\eta\|_{2, \Gamma} \\
& \leq C\left\|u_{\epsilon, \sigma}\right\|\left\|\psi_{\epsilon, \sigma}\right\|_{H^{2}(\Gamma)}^{1 / 2}\left\|\psi_{\epsilon, \sigma}\right\|_{H^{1}(\Gamma)}^{1 / 2}\|\eta\|_{2, \Gamma},
\end{aligned}
$$

so that

$$
\begin{aligned}
& \left|b_{\Gamma}^{T}\left(u_{\epsilon, \sigma}, K_{\epsilon} \psi_{\epsilon, \sigma}, \eta\right)\right|=\left|\int_{0}^{T} b_{\Gamma}\left(u_{\epsilon, \sigma}(t), K_{\epsilon} \psi_{\epsilon, \sigma}(t), \eta(t)\right) d t\right| \\
& \leq C\|\eta\|_{L^{4}\left(L^{2}(\Gamma)\right)}\left\|u_{\epsilon, \sigma}\right\|_{L^{2}\left(\mathbb{H}^{1}\right)}\left\|\psi_{\epsilon, \sigma}\right\|_{L^{2}\left(H^{2}(\Gamma)\right)}^{1 / 2}\left\|\psi_{\epsilon, \sigma}\right\|_{L^{\infty}\left(H^{1}(\Gamma)\right)}^{1 / 2} \\
& \leq C,
\end{aligned}
$$

by virtue of Lemma 4.7 and the uniform estimates (5.56) and (5.59). Therefore,

$$
B_{\Gamma}\left(u_{\epsilon, \sigma}, K_{\epsilon} \psi_{\epsilon, \sigma}\right) \text { is uniformly bounded in } L^{4 / 3}\left(0, T ; L^{2}(\Gamma)\right) \text { if } N=3 \text {. }
$$

Case $N=2$ : Let $\eta \in L^{4 s /(s+2)}\left(0, T ; L^{2}(\Gamma)\right)$, such that $\|\eta\|_{L^{4 s /(s+2)}\left(0, T ; L^{2}(\Gamma)\right)} \leq 1$ for any $s \in$ $(2, \infty)$. In that case, $H^{1 / 2}(\Gamma) \hookrightarrow L^{2 s /(s-2)}(\Gamma)$ and the Sobolev inequality

$$
\left\|\nabla_{\tau} \psi\right\|_{s, \Gamma} \lesssim\|\psi\|_{H^{2}(\Gamma)}^{\frac{s-2}{2 s}}\left\|\nabla_{\tau} \psi\right\|_{2, \Gamma}^{\frac{1}{2}+\frac{1}{s}}
$$


holds (note that $H^{1 / 2}(\Gamma) \nsubseteq L^{\infty}(\Gamma)$, so that we can not take $s=2$ ). By application of Lemma 4.7 once more, observing that

$$
\begin{aligned}
\left|b_{\Gamma}\left(u_{\epsilon, \sigma}, K_{\epsilon} \psi_{\epsilon, \sigma}, \eta\right)\right| & \leq C\left|u_{\tau \epsilon, \sigma}\right|_{2 s /(s-2), \Gamma}\left\|\nabla_{\tau} K_{\epsilon} \psi_{\epsilon, \sigma}\right\|_{s, \Gamma}\|\eta\|_{2, \Gamma} \\
& \leq C\left\|u_{\epsilon, \sigma}\right\|\left\|K_{\epsilon} \psi_{\epsilon, \sigma}\right\|_{H^{2}(\Gamma)}^{\frac{s-2}{2 s}}\left\|K_{\epsilon} \psi_{\epsilon, \sigma}\right\|_{H^{1}(\Gamma)}^{\frac{1}{2}+\frac{1}{s}}\|\eta\|_{2, \Gamma} \\
& \leq C\left\|u_{\epsilon, \sigma}\right\|\left\|\psi_{\epsilon, \sigma}\right\|_{H^{2}(\Gamma)}^{\frac{s-2}{2 s}}\left\|\psi_{\epsilon, \sigma}\right\|_{H^{1}(\Gamma)}^{\frac{1}{2}+\frac{1}{s}}\|\eta\|_{2, \Gamma},
\end{aligned}
$$

we have

$$
\begin{aligned}
& \left|b_{\Gamma}^{T}\left(u_{\epsilon}, K_{\epsilon} \psi_{\epsilon}, \eta\right)\right| \\
& \leq C\|\eta\|_{L^{4 s /(s+2)}\left(L^{2}(\Gamma)\right)}\left\|u_{\epsilon, \sigma}\right\|_{L^{2}\left(\mathbb{H}^{1}\right)}\left\|\psi_{\epsilon, \sigma}\right\|_{L^{2}\left(H^{2}(\Gamma)\right)}^{\frac{s-2}{2 s}}\left\|\psi_{\epsilon, \sigma}\right\|_{L^{\infty}\left(H^{1}(\Gamma)\right)}^{\frac{1}{2}+\frac{1}{s}} \\
& \leq C .
\end{aligned}
$$

Thus,

$$
B_{\Gamma}\left(u_{\epsilon, \sigma}, K_{\epsilon} \psi_{\epsilon, \sigma}\right) \text { is uniformly bounded in } L^{\frac{4 s}{3 s-2}}\left(0, T ; L^{2}(\Gamma)\right) \text { if } N=2 .
$$

By comparison in equation (2.8), recalling the last bound in (5.56) we see that

$$
\begin{aligned}
& \partial_{t} \psi_{\epsilon, \sigma} \text { is uniformly bounded in } L^{4 / 3}\left(0, T ; L^{2}(\Gamma)\right) \text { if } N=3, \\
& \partial_{t} \psi_{\epsilon, \sigma} \text { is uniformly bounded in } L^{\frac{4 s}{3 s-2}}\left(0, T ; L^{2}(\Gamma)\right) \text { if } N=2 .
\end{aligned}
$$

In order to prove that $\partial_{t} u_{\epsilon, \sigma}$ is properly bounded in some space, one needs to (uniformly) control the term $\mathcal{L}\left(\psi_{\epsilon, \sigma}\right) \nabla_{\tau} K_{\epsilon} \psi_{\epsilon, \sigma}$ on the right-hand side of (2.6). Since for smooth enough $v$ there holds $\left\langle\mathcal{L}\left(\psi_{\epsilon, \sigma}\right) \nabla_{\tau} K_{\epsilon} \psi_{\epsilon, \sigma}, v\right\rangle=b_{\Gamma}\left(v, K_{\epsilon} \psi_{\epsilon, \sigma}, \mathcal{L}\left(\psi_{\epsilon, \sigma}\right)\right)$, the previous considerations in (5.65)-(5.67) yield again that

$$
\mathcal{L}\left(\psi_{\epsilon, \sigma}\right) \nabla_{\tau} K_{\epsilon} \psi_{\epsilon, \sigma} \text { is uniformly bounded in } L^{4 / 3}\left(0, T ;\left(\mathbb{H}^{1}\right)^{*}\right) \text { if } N=3
$$

and, for any $s>2$,

$$
\mathcal{L}\left(\psi_{\epsilon, \sigma}\right) \nabla_{\tau} K_{\epsilon} \psi_{\epsilon, \sigma} \text { is uniformly bounded in } L^{\frac{4 s}{3 s-2}}\left(0, T ;\left(\mathbb{H}^{1}\right)^{*}\right) \text { if } N=2 .
$$

Together with the foregoing bound on $B_{0}$ (see (5.60)), by comparison in equation (2.6),

$$
\begin{aligned}
& \partial_{t} u_{\epsilon, \sigma} \text { is uniformly bounded in } L^{4 / 3}\left(0, T ;\left(\mathbb{H}^{1}\right)^{*}\right) \text { if } N=3, \\
& \partial_{t} u_{\epsilon, \sigma} \text { is uniformly bounded in } L^{\frac{4 s}{3 s-2}}\left(0, T ;\left(\mathbb{H}^{1}\right)^{*}\right) \text { if } N=2 .
\end{aligned}
$$

These bounds allow us to find a subsequence (relabeled, abusing notation, as $\left(u_{\epsilon, \sigma}, \phi_{\epsilon, \sigma}, \psi_{\epsilon, \sigma}\right)$ ) such that, as $(\epsilon, \sigma) \rightarrow(0,0)$,

$$
\left\{\begin{array}{l}
\left(u_{\epsilon, \sigma}, \phi_{\epsilon, \sigma}, \psi_{\epsilon, \sigma}\right) \rightarrow(u, \phi, \psi) \text { weakly star in } L^{\infty}\left(0, T ; \mathbb{H} \times V_{\gamma}^{1}\right), \\
\left(u_{\epsilon, \sigma}, \phi_{\epsilon, \sigma}, \psi_{\epsilon, \sigma}\right) \rightarrow(u, \phi, \psi) \text { weakly in } L^{2}\left(0, T ; \mathbb{H}^{1} \times \mathbb{V}_{\gamma}^{2}\right), \\
\partial_{t} u_{\epsilon, \sigma} \rightarrow \partial_{t} u \text { weakly in } L^{p}\left(0, T ;\left(\mathbb{H}^{1}\right)^{*}\right), \\
\partial_{t} \psi_{\epsilon, \sigma} \rightarrow \partial_{t} \psi \text { weakly in } L^{q}\left(0, T ; L^{2}(\Gamma)\right) \\
\partial_{t} \phi_{\epsilon, \sigma} \rightarrow \partial_{t} \phi \text { weakly in } L^{2}\left(0, T ;\left(H^{1}\right)^{*}\right),
\end{array}\right.
$$

with $(p, q) \in(1, \infty)^{2}$ as above. Furthermore, by (5.57) we readily have

$$
\sigma \partial_{t} \phi_{\epsilon, \sigma} \rightarrow 0 \text { strongly in } L^{2}\left(0, T ; L^{2}(\Omega)\right) \text { as }(\epsilon, \sigma) \rightarrow(0,0) \text {. }
$$

We can now exploit the compactness theorem (see Lemma 4.4) to further extract a subsequence such that

$$
\left\{\begin{array}{l}
u_{\epsilon, \sigma} \rightarrow u \text { strongly in } L^{2}\left(0, T ; \mathbb{H}^{1-\delta}\right), \\
\left(\phi_{\epsilon, \sigma}, \psi_{\epsilon, \sigma}\right) \rightarrow(\phi, \psi) \text { strongly in } L^{2}\left(0, T ; V_{\gamma}^{2-\delta}\right),
\end{array}\right.
$$

for any $\delta \in(0,1 / 2)$. The strong convergence (5.76) together with (5.74)-(5.75), the bounds (5.56)(5.58) and the fact that $f_{\epsilon} \rightarrow f, g_{\epsilon} \rightarrow f$, uniformly on compact intervals of $\mathbb{R}$, allow us to deduce that

$$
\left\{\begin{array}{l}
f_{\epsilon}\left(\phi_{\epsilon, \sigma}\right) \rightarrow f(\phi) \text { strongly in } L^{2}\left(0, T, L^{2}(\Omega)\right) \\
g_{\epsilon}\left(\psi_{\epsilon, \sigma}\right) \rightarrow g(\psi) \text { strongly in } L^{2}\left(0, T ; L^{2}(\Gamma)\right) \\
\mu_{\epsilon, \alpha} \rightarrow \mu \text { weakly in } L^{2}\left(0, T ; H^{1}(\Omega)\right) \\
\mathcal{L}_{\epsilon}\left(\psi_{\epsilon, \sigma}\right) \rightarrow \mathcal{L}(\psi) \text { weakly in } L^{2}\left(0, T ; L^{2}(\Gamma)\right)
\end{array}\right.
$$


We now show how to pass to the limit in the nonlinear boundary terms. First, from (5.76) we can assume, up to a further extraction, that $\left(u_{\epsilon, \sigma}\right)_{\tau} \rightarrow u_{\tau}$ almost everywhere in $\Gamma \times(0, T)$ and $\nabla_{\tau} \psi_{\epsilon, \sigma} \rightarrow \nabla_{\tau} \psi$ almost everywhere on $\Gamma \times(0, T)$. This gives

$$
\left(u_{\epsilon, \sigma}\right)_{\tau_{i}} \partial_{\tau_{i}} \psi_{\epsilon, \sigma} \rightarrow u_{\tau_{i}} \partial_{\tau_{i}} \psi, \quad \text { a.e. on } \Gamma \times(0, T)
$$

as $(\epsilon, \sigma) \rightarrow(0,0)$. Since $\left(u_{\epsilon, \sigma}, \psi_{\epsilon, \sigma}\right)$ is uniformly bounded according to (5.74), we conclude from (5.65) and (5.67) that $\left(u_{\epsilon, \sigma}\right)_{\tau_{i}} \partial_{\tau_{i}} \psi_{\epsilon, \sigma}$ is bounded uniformly in $L^{4 / 3}\left(0, T ; L^{2}(\Gamma)\right)$ if $N=3$ and in $L^{\frac{4 s}{3 s-2}}\left(0, T ; L^{2}(\Gamma)\right)$ if $N=2$, for any $s>2$. Therefore, $\left(u_{\epsilon, \sigma}\right)_{\tau_{i}} \partial_{\tau_{i}} \psi_{\epsilon, \sigma}$ is bounded in $L^{4 / 3}(\Gamma \times(0, T))$ if $N=3$ and in $L^{\frac{4 s}{3 s-2}}(\Gamma \times(0, T))$ if $N=2$. Applying the Lebesgue dominated convergence theorem (see Lemma 4.5) we have

$$
\left(u_{\epsilon, \sigma}\right)_{\tau_{i}} \partial_{\tau_{i}} \psi_{\epsilon, \sigma} \rightarrow u_{\tau_{i}} \partial_{\tau_{i}} \psi \text { weakly in } L^{4 / 3}(\Gamma \times(0, T)) \text { if } N=3
$$

and weakly in $L^{\frac{4 s}{3 s-2}}(\Gamma \times(0, T))$ if $N=2$. Moreover, the convergence (5.78) also holds weakly in $L^{4 / 3}\left(0, T ; L^{2}(\Gamma)\right)$ if $N=3$ and in $L^{\frac{4 s}{3 s-2}}\left(0, T ; L^{2}(\Gamma)\right)$ if $N=2$, for any $s>2$, on account of (5.65)-(5.67). Hence, we infer that

$$
\left\{\begin{array}{l}
B_{\Gamma}\left(u_{\epsilon, \sigma}, K_{\epsilon}\left(\psi_{\epsilon, \sigma}\right)\right) \rightarrow B_{\Gamma}(u, \psi) \quad \text { weakly in } L^{4 / 3}\left(0, T ; L^{2}(\Gamma)\right) \text { if } N=3, \\
B_{\Gamma}\left(u_{\epsilon, \sigma}, K_{\epsilon}\left(\psi_{\epsilon, \sigma}\right)\right) \rightarrow B_{\Gamma}(u, \psi) \quad \text { weakly in } L^{\frac{4 s}{3 s-2}}\left(0, T ; L^{2}(\Gamma)\right) \text { if } N=2,
\end{array}\right.
$$

owing to the strong convergence $K_{\epsilon}\left(\psi_{\epsilon, \sigma}\right) \rightarrow \psi$ in $H^{1}(\Gamma)$ almost everywhere on $(0, T)$ (cf. Lemma 4.1 , (iii)). It remains to pass to the limit in the boundary term (5.3). To this end, we first notice that

$$
\begin{aligned}
\mathcal{L}_{\epsilon}\left(\psi_{\epsilon, \sigma}\right) \nabla_{\tau} \psi_{\epsilon, \sigma} & =\left(-\gamma A_{\tau} \psi_{\epsilon, \sigma}+\partial_{n} \phi_{\epsilon, \sigma}+\zeta \psi_{\epsilon, \sigma}+g_{\epsilon}\left(\psi_{\epsilon, \sigma}\right)\right) \nabla_{\tau} \psi_{\epsilon, \sigma} \\
& =I_{\epsilon \sigma}^{1}+I_{\epsilon \sigma}^{2}+I_{\epsilon \sigma}^{3} .
\end{aligned}
$$

For the last two terms, we can argue as above on account of the strong convergence properties of (5.76) and the second of (5.77) to deduce that $I_{\epsilon \sigma}^{2} \rightarrow \partial_{n} \phi \nabla_{\tau} \psi$ almost everywhere on $\Gamma \times(0, T)$ and $I_{\epsilon \sigma}^{3} \rightarrow(\zeta \psi+g(\psi)) \nabla_{\tau} \psi$, almost everywhere on $\Gamma \times(0, T)$, as $(\epsilon, \sigma) \rightarrow(0,0)$. Indeed, owing once again to the Lebesgue theorem (see Lemma 4.5), combined with the same arguments leading to (5.70) and (5.71), we see that $I_{\epsilon \sigma}^{2}$ and $I_{\epsilon \sigma}^{3}$ are uniformly bounded in $L^{4 / 3}\left(0, T ;\left(\mathbb{H}^{1}\right)^{*}\right)$ if $N=3$ and in $L^{\frac{4 s}{3 s-2}}\left(0, T ;\left(\mathbb{H}^{1}\right)^{*}\right)$ when $N=2$. Hence, we get

$$
I_{\epsilon \sigma}^{2} \rightarrow \partial_{n} \phi \nabla_{\tau} \psi, I_{\epsilon \sigma}^{3} \rightarrow(\zeta \psi+g(\psi)) \nabla_{\tau} \psi \text {, weakly in }\left\{\begin{array}{c}
L^{4 / 3}\left(0, T ;\left(\mathbb{H}^{1}\right)^{*}\right) \text { if } N=3, \\
L^{\frac{4 s}{3 s-2}}\left(0, T ;\left(\mathbb{H}^{1}\right)^{*}\right) \text { if } N=2 .
\end{array}\right.
$$

On the other hand, to handle the first term $I_{\epsilon \sigma}^{1}$, we exploit the basic identity

$$
I_{\epsilon \sigma}^{1}=-\gamma A_{\tau} \psi_{\epsilon, \sigma} \nabla_{\tau} \psi_{\epsilon, \sigma}=\frac{\gamma}{2} \nabla_{\tau}\left(\left|\nabla_{\tau} \psi_{\epsilon, \sigma}\right|^{2}\right)-\gamma \operatorname{div}_{\tau}\left(\nabla_{\tau} \psi_{\varepsilon, \sigma} \otimes \nabla_{\tau} \psi_{\varepsilon, \sigma}\right),
$$

where $\operatorname{div}_{\tau}(\cdot)$ stands for the boundary divergence operator. By (5.76), we may assume

$$
\partial_{\tau_{i}} \psi_{\varepsilon, \sigma} \partial_{\tau_{j}} \psi_{\varepsilon, \sigma} \rightarrow \partial_{\tau_{i}} \psi \partial_{\tau_{j}} \psi, \quad \text { a.e. on } \Gamma \times(0, T) \text {, }
$$

for any $1 \leq i, j \leq N-1$. Since $\left\{\psi_{\epsilon, \sigma}\right\}$ is also uniformly bounded in $L^{\infty}\left(0, T ; H^{1}(\Gamma)\right) \cap$ $L^{2}\left(0, T ; H^{2}(\Gamma)\right)$ and each term $\partial_{\tau_{i}} \psi_{\epsilon, \sigma} \partial_{\tau_{j}} \psi_{\epsilon, \sigma}(1 \leq i, j \leq N-1)$ is bounded in $H^{1 / 2}(\Gamma)$ as a product of functions in $H^{\alpha_{N}}(\Gamma) \times H^{1}(\Gamma) \rightarrow H^{1 / 2}(\Gamma)$, by Lemma 4.8, with

$$
\alpha_{3}=1 / 2+\delta, \quad \alpha_{2}=\frac{s-2}{2 s}
$$

for any $s \in(2, \infty)$ and $\delta \in(0,1 / 2)$, we conclude by interpolation between $H^{1}(\Gamma) \hookrightarrow H^{\alpha_{N}}(\Gamma) \hookrightarrow$ $L^{2}(\Gamma)$ that

$$
\begin{aligned}
& \partial_{\tau_{i}} \psi_{\epsilon, \sigma} \partial_{\tau_{j}} \psi_{\epsilon, \sigma} \in L^{\frac{4}{3+2 \delta}}\left(0, T ; H^{1 / 2}(\Gamma)\right) \text { if } N=3, \\
& \partial_{\tau_{i}} \psi_{\epsilon, \sigma} \partial_{\tau_{j}} \psi_{\epsilon, \sigma} \in L^{\frac{4 s}{3 s-2}}\left(0, T ; H^{1 / 2}(\Gamma)\right) \text { if } N=2 .
\end{aligned}
$$

Therefore, owing to the Stokes formula on $\Gamma,\left(\operatorname{div}_{\tau}(\mathbb{D}), v_{\tau}\right)=-\left(\mathbb{D}, \nabla_{\tau} v_{\tau}\right)_{\Gamma}$, which holds for smooth $\mathbb{D} \in \mathbb{R}^{N \times N}, v_{\tau}=v-(v \cdot n) n$ being the tangential part of $v \in \mathbb{R}^{N}$, the (distributional) 
boundary divergence of $\nabla_{\tau} \psi_{\varepsilon, \sigma} \otimes \nabla_{\tau} \psi_{\varepsilon, \sigma}$ satisfies

$$
\operatorname{div}_{\tau}\left(\nabla_{\tau} \psi_{\varepsilon, \sigma} \otimes \nabla_{\tau} \psi_{\varepsilon, \sigma}\right) \in \begin{cases}L^{\frac{4}{3+2 \delta}}\left(0, T ;\left(\mathbb{H}^{1 / 2}(\Gamma)\right)^{*}\right) & \text { if } N=3, \\ L^{\frac{4 s}{3 s-2}}\left(0, T ;\left(\mathbb{H}^{1 / 2}(\Gamma)\right)^{*}\right) & \text { if } N=2 .\end{cases}
$$

Then, as a consequence, $\operatorname{div}_{\tau}\left(\nabla_{\tau} \psi_{\varepsilon, \sigma} \otimes \nabla_{\tau} \psi_{\varepsilon, \sigma}\right)$ is uniformly bounded in $L^{\frac{4}{3+2 \delta}}\left(0, T ; \mathbb{H}^{-1}\right)$ if $N=3$ and in $L^{\frac{4 s}{3 s-2}}\left(0, T ; \mathbb{H}^{-1}\right)$ if $N=2$. Henceforth, the application of Lemma 4.5 yields the weak convergence of

$$
\operatorname{div}_{\tau}\left(\nabla_{\tau} \psi_{\varepsilon, \sigma} \otimes \nabla_{\tau} \psi_{\varepsilon, \sigma}\right) \rightarrow \operatorname{div}_{\tau}\left(\nabla_{\tau} \psi \otimes \nabla_{\tau} \psi\right)
$$

on these spaces (see (5.83)). Arguing in a similar fashion, it follows that $\left|\nabla_{\tau} \psi_{\epsilon, \sigma}\right|^{2}$ is also uniformly bounded in $L^{\frac{4}{3+2 \delta}}\left(0, T ; H^{1 / 2}(\Gamma)\right)$ if $N=3$ and in $L^{\frac{4 s}{3 s-2}}\left(0, T ; H^{-1 / 2}(\Gamma)\right)$ if $N=2$. Thus $\nabla_{\tau}\left(\left|\nabla_{\tau} \psi_{\epsilon, \sigma}\right|^{2}\right) \rightarrow \nabla_{\tau}\left(\left|\nabla_{\tau} \psi\right|^{2}\right)$ weakly converges in the spaces defined in $(5.83)$, as $(\epsilon, \sigma) \rightarrow(0,0)$. Hence, on the basis of (5.82) we conclude that

$$
\begin{aligned}
& I_{\epsilon, \sigma}^{1} \rightarrow-\gamma A_{\tau} \psi \nabla_{\tau} \psi \text { weakly in } L^{\frac{4}{3+2 \delta}}\left(0, T ;\left(\mathbb{H}^{1}\right)^{*}\right) \text { if } N=3, \\
& I_{\epsilon, \sigma}^{1} \rightarrow-\gamma A_{\tau} \psi \nabla_{\tau} \psi \text { weakly in } L^{\frac{4 s}{3 s-2}}\left(0, T ;\left(\mathbb{H}^{1}\right)^{*}\right) \text { if } N=2 .
\end{aligned}
$$

Since $4 /(3+2 \delta)<4 / 3$ when $N=3$, this entails that

$$
\mathcal{L}_{\epsilon}\left(\psi_{\epsilon, \sigma}\right) \nabla_{\tau} \psi_{\epsilon, \sigma} \rightarrow \mathcal{L}(\psi) \nabla_{\tau} \psi \text { weakly in } L^{\frac{4}{3+2 \delta}}\left(0, T ;\left(\mathbb{H}^{1}\right)^{*}\right) \text { if } N=3
$$

and also in $L^{\frac{4 s}{3 s-2}}\left(0, T ;\left(\mathbb{H}^{1}\right)^{*}\right)$ when $N=2$. Recalling now that $K_{\epsilon}\left(\psi_{\epsilon, \sigma}\right) \rightarrow \psi$ strongly in $H^{1}(\Gamma)$ almost everywhere in $(0, T)$, owing to Lemma 4.1 , (iii), we also obtain an analogous weak convergence of $\mathcal{L}_{\epsilon}\left(\psi_{\epsilon, \sigma}\right) \nabla_{\tau}\left(K_{\epsilon} \psi_{\epsilon, \sigma}\right)$ to $\mathcal{L}(\psi) \nabla_{\tau} \psi$.

The same arguments based on the strong convergence (5.76) and the uniform bounds (5.56) gives us the weak convergence of the remaining nonlinear terms $B_{0}, B_{1}$ in $L^{p}\left(0, T ;\left(\mathbb{H}^{1}\right)^{*}\right)$ and $L^{2}\left(0, T ;\left(H^{1}\right)^{*}\right)$, respectively. More precisely, we have

$$
\begin{gathered}
B_{0}\left(J_{\epsilon}\left(u_{\epsilon, \sigma}\right), u_{\epsilon, \sigma}\right) \rightarrow B_{0}(u, u) \text { weakly in } L^{p}\left(0, T ;\left(\mathbb{H}^{1}\right)^{*}\right), \\
B_{1}\left(u_{\epsilon, \sigma}, J_{\epsilon}\left(\phi_{\epsilon, \sigma}\right)\right) \rightarrow B_{1}(u, \phi) \text { weakly in } L^{2}\left(0, T ;\left(H^{1}\right)^{*}\right)
\end{gathered}
$$

and, similarly, we infer that

$$
\mu_{\epsilon, \sigma} \nabla\left(J_{\epsilon} \phi_{\epsilon, \sigma}\right) \rightarrow \mu \nabla \phi \text { weakly in } L^{p}\left(0, T ;\left(\mathbb{H}^{1}\right)^{*}\right)
$$

since

$$
\sigma \partial_{t} \phi_{\epsilon, \sigma} \nabla J_{\epsilon} \phi_{\epsilon, \sigma} \rightarrow 0 \text { strongly in } L^{p}\left(0, T ;\left(\mathbb{H}^{1}\right)^{*}\right)
$$

and

$$
\operatorname{div}\left(\nabla \phi_{\epsilon} \otimes \nabla J_{\epsilon} \phi_{\epsilon}\right) \rightarrow \operatorname{div}(\nabla \phi \otimes \nabla \phi) \text { weakly in } L^{p}\left(0, T ;\left(\mathbb{H}^{1}\right)^{*}\right)
$$

as $(\epsilon, \sigma) \rightarrow(0,0)$, owing to the strong convergence of the sequence $\left(u_{\epsilon}, \phi_{\epsilon}\right)$ (see $\left.(5.76)\right),(5.75)$, Lemma 4.1, (iii) (which yields $J_{\epsilon} \phi_{\epsilon} \rightarrow \phi$ strongly in $H^{1}(\Omega)$ almost everywhere in $(0, T)$ ) and Lemma 4.5.

We can now pass to the limit in (5.3)-(5.7) with respect to $(\epsilon, \sigma) \rightarrow(0,0)$ in order to deduce that the limit function $(u, \phi, \psi)$ obtained from (5.74)-(5.76) is a weak solution to problem (1.1)-(1.10) in the sense of Definition 2.1. Besides, by virtue of Remark 2.1, for $t \in[0, T]$,

$$
\left(u_{0}, \phi_{0}, \psi_{0}\right)=\left(u_{\epsilon, \sigma}(t), \phi_{\epsilon, \sigma}(t), \psi_{\epsilon, \sigma}(t)\right)_{\mid t=0} \rightarrow\left(u_{0}, \phi_{0}, \psi_{0}\right)=(u(t), \phi(t), \psi(t))_{\mid t=0}
$$

weakly in $\left(\mathbb{H}^{1}\right)^{*} \times V_{\gamma}^{0}$ as $(\epsilon, \sigma) \rightarrow(0,0)$. In order to see that the energy inequality (3.6) holds for the limit weak solution $(u, \phi, \psi)$, it is enough to notice that it is also satisfied by the approximate solution $\left(u_{\epsilon, \sigma}, \phi_{\epsilon, \sigma}, \psi_{\epsilon, \sigma}\right)$, according to (5.55), then to exploit the lower-semicontinuity of the norm in $\mathbb{Y}_{\gamma}$ and (5.75) together with the basic relations

$$
\left\{\begin{aligned}
\int_{\Omega} F(\phi(t)) d x & \leq \liminf _{(\epsilon, \sigma) \rightarrow(0,0)} \int_{\Omega} F_{\epsilon}\left(\phi_{\epsilon, \sigma}(t)\right) d x \\
\int_{\Omega} G(\psi(t)) d S & \leq \liminf _{(\epsilon, \sigma) \rightarrow(0,0)} \int_{\Gamma} G_{\epsilon}\left(\psi_{\epsilon, \sigma}(t)\right) d S,
\end{aligned}\right.
$$

which hold owing to Fatou's lemma, assumption (3.1) and (5.76). Therefore $(u, \phi, \psi)$ is a solution in the sense of Definition 3.1.

We are left to prove that our energy solution can indeed satisfy the inequality (3.8) in the case $h \equiv 0$. The claim for $s=0$ follows from Definition 3.1 (see also (3.7)). Let us briefly show how to 
prove it for $t \geq s$ for almost all $s \in(0, \infty)$. We can exploit the smooth solutions $\left(u_{\epsilon, \sigma}, \phi_{\epsilon, \sigma}, \psi_{\epsilon, \sigma}\right)$ for which the energy inequality

$$
\frac{d}{d t} E_{\epsilon, \sigma}(t)+a_{0}\left(u_{\epsilon, \sigma}(t), u_{\epsilon, \sigma}(t)\right)+\left\|\nabla \mu_{\epsilon, \sigma}(t)\right\|_{2}^{2}+\left\|\mathcal{L}\left(\psi_{\epsilon, \sigma}(t)\right)\right\|_{2, \Gamma}^{2}+\sigma\left\|\partial_{t} \phi_{\epsilon, \sigma}(t)\right\|_{2}^{2} \leq 0
$$

is satisfied for any $t \geq s \geq 0$. In fact, we can readily see from the proof of Theorem 5.1 that, for these approximate solutions, (5.87) becomes an identity for any $t \geq 0$. In order to obtain the final claim, one passes to the limit as $(\epsilon, \sigma) \rightarrow(0,0)$ in the integrated version of this identity between $(s, t)$ and exploits the uniform bounds for $\left(u_{\epsilon, \sigma}, \phi_{\epsilon, \sigma}, \psi_{\epsilon, \sigma}\right)$ obtained in the proof of Theorem 3.2. In particular, by the first two convergence (5.74) and the second of (5.76), it follows by interpolation that, for almost any $s>0, \phi_{\epsilon, \sigma}(s) \rightarrow \phi(s)$ strongly in $L^{l}(\Omega), \psi_{\epsilon, \sigma}(s) \rightarrow \psi(s)$ strongly in $L^{s}(\Gamma)$, at least along a subsequence for sufficiently large $l, s>2$. This fact together with the growth assumptions on $f, g$ (see (3.2)) and the uniform convergences $F_{\epsilon} \rightarrow F$ and $G_{\epsilon} \rightarrow G$ on compact intervals allow us to deduce by the Lebesgue theorem that

$$
\int_{\Omega} F_{\epsilon}\left(\phi_{\epsilon, \sigma}(s)\right) d x \rightarrow \int_{\Omega} F(\phi(s)) d x, \int_{\Gamma} G_{\epsilon}\left(\psi_{\epsilon, \sigma}(s)\right) d S \rightarrow \int_{\Gamma} G(\psi(s)) d S,
$$

for almost any $s>0$. Finally, these relations together with (5.75) and the lower-semicontinuity of the total energy (note that both $F$ and $G$ are quadratic perturbations of some convex functions due to (3.1)) yield the desired claim.

This completes the proof of Theorem 3.2.

Remark 5.3. If we keep $\sigma>0$ fixed in the above proof, we may also conclude the existence of a weak solution $\left(u_{\sigma}, \phi_{\sigma}, \psi_{\sigma}\right)$ for our system (1.1)-(1.10) in the sense of Definition 2.1. This solution enjoys better integrability properties under the assumptions of Theorem 3.2. In particular, one has $\sigma^{1 / 2} \partial_{t} \phi_{\sigma} \in L^{2}\left(0, T ; L^{2}(\Omega)\right)$ and

$$
B_{1}\left(u_{\sigma}, \varphi_{\sigma}\right) \in L^{2}\left(0, T ; L^{2}(\Omega)\right), \mu_{\sigma} \in L^{2}\left(0, T ; D\left(A_{N}\right)\right),
$$

and the following (strong) energy inequality if $h \equiv 0$,

$$
\begin{aligned}
& \mathcal{E}\left(u_{\sigma}(t), \phi_{\sigma}(t), \psi_{\sigma}(t)\right) \\
& +\int_{s}^{t}\left(a_{0}\left(u_{\sigma}(y), u_{\sigma}(y)\right)+\left\|\nabla \mu_{\sigma}(y)\right\|_{2}^{2}+\left\|\mathcal{L}\left(\psi_{\sigma}(y)\right)\right\|_{2, \Gamma}^{2}+\sigma\left\|\partial_{t} \phi_{\sigma}(y)\right\|_{2}^{2}\right) d y \\
& \leq \mathcal{E}\left(u_{\sigma}(s), \phi_{\sigma}(s), \psi_{\sigma}(s)\right),
\end{aligned}
$$

for all $t \geq s$ and for almost any $s \in[0, \infty)$, including $s=0$.

\section{Proof of Theorem 3.3}

We first prove that every energy solution given by Definition 3.1 has a non-empty $\omega$-limit set.

Lemma 6.1. Let the assumptions of Theorem 3.2 be satisfied and suppose that $h \equiv 0$. Let $(u, \phi, \psi)$ be an energy solution. Then the $\omega$-limit set of $(u, \phi, \psi)$ is nonempty. More precisely, we have

$$
\lim _{t \rightarrow \infty} u(t)=0 \text { weakly in } \mathbb{H} \text {, strongly in }\left(\mathbb{H}^{1}\right)^{*}
$$

and any unbounded sequence $\left\{t_{n}\right\} \subset[0, \infty)$ admits a subsequence, denoted by $\left\{t_{n_{k}}\right\}$, such that

$$
\lim _{t_{n_{k}} \rightarrow \infty}\left(\phi\left(t_{n_{k}}\right), \psi\left(t_{n_{k}}\right)\right)=\left(\phi_{*}, \psi_{*}\right) \text { weakly in } V_{\gamma}^{1} \text {, strongly in } V_{\gamma}^{0} \text {, }
$$

for some $\left(\phi_{*}, \psi_{*}\right) \in V_{\gamma}^{2} \cap\left\{\phi_{*}:\left\langle\phi_{*}\right\rangle=\left\langle\phi_{0}\right\rangle\right\}$ such that

$$
-\Delta \phi_{*}+f\left(\phi_{*}\right)=\text { const., in } \Omega, \quad-\gamma A_{\tau} \psi_{*}+\partial_{n} \phi_{*}+\zeta \psi_{*}+g\left(\psi_{*}\right)=0 \text {, on } \Gamma \text {. }
$$

Proof. We argue as in the proof of Theorem 3.2 which ensures the existence of an energy solution $(u, \phi, \psi)$. Let $\left\{t_{n}\right\} \subset[0, \infty)$ be a unbounded sequence. The energy inequality (3.7) implies, at least for a suitable subsequence of $\left\{t_{n}\right\}$, still labeled as $\left\{t_{n}\right\}$, that

$$
u\left(t_{n}\right) \rightarrow u_{*} \text { weakly in } \mathbb{H}, \quad(\phi, \psi)\left(t_{n}\right) \rightarrow\left(\phi_{*}, \psi_{*}\right) \text { weakly in } V_{\gamma}^{1},
$$

for some $\left(\phi_{*}, \psi_{*}\right) \in V_{\gamma}^{1}$. Consider now the initial value problem associated with (1.1)-(1.9) on the time interval $\left[t_{n}, t_{n+1}\right]$ with the initial values $\left(u\left(t_{n}\right), \phi\left(t_{n}\right), \psi\left(t_{n}\right)\right)$ and observe that 
$\left(u_{n}(t), \phi_{n}(t), \psi_{n}(t)\right):=\left(u\left(t+t_{n}\right), \phi\left(t+t_{n}\right), \psi\left(t+t_{n}\right)\right)$ is also a weak solution to (1.1)-(1.9) with $\left\langle\phi_{n}(t)\right\rangle=\left\langle\phi_{0}\right\rangle$ for $t \in[0,1]$. Then, from (3.7) we also infer, as $t_{n} \rightarrow \infty$, that

$$
\left\{\begin{array}{l}
u_{n} \rightarrow 0 \text { strongly in } L^{2}\left(0,1 ; \mathbb{H}^{1}\right) \text {, weakly star in } L^{\infty}(0,1 ; \mathbb{H}), \\
\left(\phi_{n}, \psi_{n}\right) \rightarrow(\bar{\phi}, \bar{\psi}) \text { weakly in } L^{2}\left(0,1 ; V_{\gamma}^{2}\right) \text {, weakly star in } L^{\infty}\left(0,1 ; V_{\gamma}^{1}\right),
\end{array}\right.
$$

for a suitable $(\bar{\phi}, \bar{\psi})$. Repeating the comparison arguments used in the proof of Theorem 3.2, we deduce that

$$
\partial_{t} \phi_{n} \rightarrow \partial_{t} \bar{\phi} \text { weakly in } L^{2}\left(0,1 ;\left(H^{1}(\Omega)\right)^{*}\right), \quad \partial_{t} \psi_{n} \rightarrow \partial_{t} \bar{\psi} \text { weakly in } L^{q}\left(0,1 ; L^{2}(\Gamma)\right)
$$

and, in particular, we see that $\partial_{t} u_{n}$ is uniformly bounded in $L^{p}\left(0,1 ;\left(\mathbb{H}^{1}(\Omega)\right)^{*}\right)$, for some $p>1$. Therefore, by Lemma 4.4 and (6.4)-(6.5), we obtain

$$
\begin{aligned}
& \phi_{n} \rightarrow \bar{\phi} \text { strongly in } L^{2}\left(0,1 ; H^{1}(\Omega)\right) \cap C\left([0,1] ;\left(H^{1}(\Omega)\right)^{*}\right), \\
& \psi_{n} \rightarrow \bar{\psi} \text { strongly in } L^{2}\left(0,1 ; H^{1}(\Gamma)\right) \cap C\left([0,1] ; L^{2}(\Gamma)\right),
\end{aligned}
$$

as well as

$$
u_{n} \rightarrow 0 \text { strongly in } C\left([0,1] ;\left(\mathbb{H}^{1}\right)^{*}\right) \text {. }
$$

Thus, (6.8) yields that $u_{*}=0$. This implies (6.1), in view of (6.4) and (6.8). On the other hand, by the energy inequality (3.6), we also have

$$
\left\{\begin{array}{l}
\nabla\left(-\Delta \phi_{n}+f\left(\phi_{n}\right)\right) \rightarrow 0 \text { strongly in } L^{2}\left(0,1 ;\left(L^{2}(\Omega)\right)^{N}\right), \\
-\gamma A_{\tau} \psi_{n}+\partial_{n} \phi_{n}+\zeta \psi_{n}+g\left(\psi_{n}\right) \rightarrow 0 \text { strongly in } L^{2}\left(0,1 ; L^{2}(\Gamma)\right) .
\end{array}\right.
$$

Next, by the estimates (6.4), (6.6)-(6.8), and observing that the bilinear form $B_{1}: \mathbb{H} \times L^{2} \rightarrow$ $\left(H^{2}(\Omega)\right)^{*}$ is bounded if $N \leq 3$, we also infer

$$
B_{1}\left(u_{n}, \phi_{n}\right) \rightarrow 0 \text { strongly in } L^{2}\left(0,1 ;\left(H^{2}(\Omega)\right)^{*}\right) .
$$

Thus, comparing terms in equation (2.7), we obtain

$$
\partial_{t} \phi_{n} \rightarrow 0 \text { strongly in } L^{2}\left(0,1 ;\left(H^{2}(\Omega)\right)^{*}\right) .
$$

A similar argument shows that

$$
B_{\Gamma}\left(u_{n}, \psi_{n}\right) \rightarrow 0 \text { strongly in } L^{2}\left(0,1 ;\left(H^{2}(\Gamma)\right)^{*}\right),
$$

owing to $(6.4),(6.6)$ and the fact that the terms $\left(u_{n}\right)_{\tau_{i}} \partial_{j} \psi_{n}$ are bounded in $\left(H^{2}(\Gamma)\right)^{*}$ as a product of functions in $L^{2}(\Gamma) \times L^{2}(\Gamma)$ if $N \leq 3$. Note that (6.12) entails by comparison in (2.8) and (6.9) that

$$
\partial_{t} \psi_{n} \rightarrow 0 \text { strongly in } L^{2}\left(0,1 ;\left(H^{2}(\Gamma)\right)^{*}\right) \text {. }
$$

Henceforth, it follows from (6.5) that $\bar{\phi}=\phi_{*}, \bar{\psi}=\psi_{*}$ for all $t \in[0,1]$ and $\left\langle\phi_{*}\right\rangle=\left\langle\phi_{0}\right\rangle$. Letting now $t_{n} \rightarrow \infty$ in equations (2.7)-(2.9) for $\left(u_{n}, \phi_{n}, \psi_{n}\right)$, we see that $\left(\phi_{*}, \psi_{*}\right) \in V_{\gamma}^{1}$ is also a solution to $(6.3)$, as desired. Moreover, $\left(\phi_{*}, \psi_{*}\right) \in V_{\gamma}^{2}$, by virtue of the assumptions on $f, g$, the regularity (6.4) (which themselves imply that $f\left(\phi_{*}\right) \in L^{2}(\Omega), g\left(\psi_{*}\right) \in L^{2}(\Gamma)$ ) and elliptic regularity (see Lemma 4.6). Lemma 6.1 is proved.

In general, we cannot conclude that each energy solution of (1.1)-(1.9) converges to a single equilibrium as the set of steady states associated with (6.3) can have complicated structure (see, for instance, $[4,60,74])$. This means that we are required to prove $(6.2)$ for the whole sequence $\left\{t_{n}\right\}$ and not just for a subsequence. The main tool is the total energy (cf. (3.3)) which we rewrite as follows:

$$
\begin{aligned}
\mathcal{E}(u, \phi, \psi) & :=\frac{1}{2}|u|^{2}+\widehat{\mathcal{E}}(\phi, \psi), \\
\widehat{\mathcal{E}}(\phi, \psi) & :=\frac{1}{2}\|(\phi, \psi)\|_{V_{\gamma}^{1}}^{2}+\int_{\Omega} F(\phi) d x+\int_{\Gamma} G(\psi) d S .
\end{aligned}
$$

We note that $\left(\phi_{*}, \psi_{*}\right)$ is a critical point of $\widehat{\mathcal{E}}$ over $V_{\gamma}^{1}$.

The version of the Eojasiewicz-Simon inequality we need is given by the following lemma (see [66, Theorem 3.1], cf. also [32, Proposition 5.22]). 
Lemma 6.2. Suppose that $F$ and $G$ satisfy (3.1)-(3.2) and are real analytic. Then there exist constants $\theta \in(0,1 / 2)$ and $C_{L}>0, \eta>0$ depending on $\left(\phi_{*}, \psi_{*}\right)$ such that, for any $(\phi, \psi) \in V_{\gamma}^{1}$, if $\left\|(\phi, \psi)-\left(\phi_{*}, \psi_{*}\right)\right\|_{V_{\gamma}^{1}} \leq \eta$, denoting by $\widehat{\mathcal{E}}^{\prime}$ the Fréchet derivative of $\widehat{\mathcal{E}}$, we have

$$
C_{L}|| \widehat{\mathcal{E}}^{\prime}(\phi, \psi) \|_{\left(V_{\gamma}^{1}\right)^{*}} \geq\left|\widehat{\mathcal{E}}(\phi, \psi)-\widehat{\mathcal{E}}\left(\phi_{*}, \psi_{*}\right)\right|^{1-\theta} .
$$

The following statement is valid for any energy solution $(u, \phi, \psi)$ which satisfies (3.8). Indeed (6.16) below follows from this, the fact that $\widehat{\mathcal{E}}$ is bounded from below on $V_{\gamma}^{1}$ and (6.1)-(6.3).

Proposition 6.3. There exists a constant $e_{*} \in \mathbb{R}$ with $\widehat{\mathcal{E}}\left(\phi_{*}, \psi_{*}\right)=e_{*}$, for all solutions $\left(\phi_{*}, \psi_{*}\right)$ satisfying (6.3), such that

$$
\lim _{t \rightarrow \infty} \mathcal{E}(u(t), \phi(t), \psi(t))=e_{*} .
$$

Remark 6.1. Set $\mathcal{M}(\phi):=-\Delta \phi+f(\phi), \mathcal{N}(\psi):=\gamma A_{\tau} \psi+\partial_{n} \phi+\zeta \psi+g(\psi)$, and observe from (6.14) that $\left\|\widehat{\mathcal{E}}^{\prime}(\phi, \psi)\right\|_{\left(V_{\gamma}^{1}\right)^{*}}=\|(\mathcal{M}(\phi), \mathcal{N}(\psi))\|_{\left(V_{\gamma}^{1}\right)^{*}}$. We emphasize that the Eojasiewicz-Simon inequality (6.15) also holds for any $(\phi, \psi) \in V_{\gamma}^{1}$ such that $\left|\widehat{\mathcal{E}}(\phi, \psi)-\widehat{\mathcal{E}}\left(\phi_{*}, \psi_{*}\right)\right| \leq C_{*}$ if

$$
\left\|(\phi, \psi)-\left(\phi_{*}, \psi_{*}\right)\right\|_{V_{\gamma}^{0}} \leq \bar{\eta},
$$

for some constants $C_{*}=C_{*}\left(e_{*}\right), \bar{\eta}>0$. Indeed, suppose not. Then, for a sequence $\left(\phi_{j}, \psi_{j}\right)$ which is bounded in $V_{\gamma}^{1}$ uniformly with respect $j \in \mathbb{N}$, such that $\left|\widehat{\mathcal{E}}\left(\phi_{j}, \psi_{j}\right)-\widehat{\mathcal{E}}\left(\phi_{*}, \psi_{*}\right)\right| \leq C_{*}$ and $\left(\phi_{j}, \psi_{j}\right) \rightarrow\left(\phi_{*}, \psi_{*}\right)$ in $V_{\gamma}^{0}$, it holds $j\left\|\widehat{\mathcal{E}}^{\prime}\left(\phi_{j}, \psi_{j}\right)\right\|_{\left(V_{\gamma}^{1}\right)^{*}} \leq\left|\widehat{\mathcal{E}}\left(\phi_{j}, \psi_{j}\right)-\widehat{\mathcal{E}}\left(\phi_{*}, \psi_{*}\right)\right|^{1-\theta}$, for all $j \in \mathbb{N}$. Thus, owing to the assumptions on $f, g$, we have as $j \rightarrow \infty$ that $\left(f\left(\phi_{j}\right), g\left(\psi_{j}\right)\right) \rightarrow\left(f\left(\phi_{*}\right), g\left(\psi_{*}\right)\right)$ in $\left(V_{\gamma}^{1}\right)^{*}$, as well as $\left(-\Delta \phi_{j}, \gamma A_{\tau} \psi_{j}+\partial_{n} \phi_{j}+\zeta \psi_{j}\right) \rightarrow\left(-\Delta \phi_{*}, \gamma A_{\tau} \psi_{*}+\partial_{n} \phi_{*}+\zeta \psi_{*}\right)$ strongly in the $\left(V_{\gamma}^{1}\right)^{*}$-norm. In particular, the latter also yields that $\left(\nabla \phi_{j}, \gamma \nabla_{\tau} \psi_{j}\right) \rightarrow\left(\nabla \phi_{*}, \gamma \nabla_{\tau} \psi_{*}\right)$ in $V_{\gamma}^{0}$, and then $\left(\phi_{j}, \psi_{j}\right) \rightarrow\left(\phi_{*}, \psi_{*}\right)$ strongly in $V_{\gamma}^{1}$ as well. The application of Lemma 6.2 with $(\phi, \psi)=\left(\phi_{j}, \psi_{j}\right)$ yields then a contradiction as soon as $j$ is large enough such that $j \geq 2 C_{L}$. Note that the norm in (6.17) can be also replaced by the $V_{\gamma}^{1-}{ }^{-}$norm owing to the boundedness of $(\phi, \psi)$, $\left(\phi_{*}, \psi_{*}\right)$ in $V_{\gamma}^{1}$ and by interpolation.

The main result of this section is concerned with the convergence of energy solutions of problem (1.1)-(1.9) to single equilibria, that is, their $\omega$-limit set is always a singleton.

Proof of Theorem 3.3. The claim in (3.10) follows from (6.1). By Proposition 6.3, the function $t \mapsto \mathcal{E}(u(t), \phi(t), \psi(t))$ is nonincreasing and (6.16) holds. Moreover, thanks to (3.8) and (6.1)(6.3), we deduce that

$$
\begin{aligned}
& \int_{0}^{\infty}\left(a_{0}(u(s), u(s))+\|\nabla \mu(s)\|_{2}^{2}+\|\mathcal{L}(\psi(s))\|_{2, \Gamma}^{2}\right) d s \\
& \leq \mathcal{E}\left(u_{0}, \phi_{0}, \psi_{0}\right)-e_{*}<\infty,
\end{aligned}
$$

since $\left(u_{0}, \phi_{0}, \psi_{0}\right) \in \mathbb{Y}_{\gamma}$. Furthermore, (6.18) and the energy inequality (3.6) again entail that

$$
\left\{\begin{array}{l}
u \in L^{2}\left(0, \infty ; \mathbb{H}^{1}\right) \cap L^{\infty}(0, \infty ; \mathbb{H}) \\
\phi \in L^{\infty}\left(0, \infty ; H^{1}(\Omega)\right), \psi \in L^{\infty}\left(0, \infty ; H^{1}(\Gamma)\right), \\
\nabla \mu \in L^{2}\left(0, \infty ;\left(L^{2}(\Omega)\right)^{N}\right) \\
\mathcal{L}(\psi) \in L^{2}\left(0, \infty ; L^{2}(\Gamma)\right)
\end{array}\right.
$$

These relations (6.19) imply that

$$
B_{1}(u, \phi) \in L^{2}\left(0, \infty ;\left(H^{1}(\Omega)\right)^{*}\right), B_{\Gamma}(u, \psi) \in L^{2}\left(0, \infty ;\left(H^{1}(\Gamma)\right)^{*}\right)
$$

and

$$
\left(B_{1}, B_{\Gamma}\right) \in L^{2}\left(0, \infty ;\left(V_{\gamma}^{1}\right)^{*}\right),
$$

which together with the last two bounds of (6.19) yield

$$
\left(\partial_{t} \phi, \partial_{t} \psi\right) \in L^{2}\left(0, \infty ;\left(V_{\gamma}^{1}\right)^{*}\right),
$$

owing to equations (2.7)-(2.8). To prove the claim in (3.9), it suffices to show that $\left(\partial_{t} \phi, \partial_{t} \psi\right) \in$ $L^{1}\left(\delta_{*}, \infty ;\left(V_{\gamma}^{1}\right)^{*}\right)$, for some $\delta_{*}>0$. Indeed, owing to the precompactness of the trajectory $(\phi, \psi)$ in $V_{\gamma}^{0}$ and by exploiting an interpolation inequality between $H^{1}(\Omega) \subset H^{1-}(\Omega) \subset L^{2}(\Omega)$, we can 
then conclude the proof of the theorem. Therefore, in order to show our last claim, we start by first observing that one can find a constant $C>0$ such that

$$
|u(t)|^{2} \leq C\|u(t)\|^{\frac{1}{1-\theta}} \leq C\left[a_{0}(u(t), u(t))^{\frac{1}{1-\theta}}, \quad \text { for a.e. } t>0,\right.
$$

which holds thanks to the embedding $\mathbb{H}^{1} \hookrightarrow \mathbb{H}$, the first relation of (6.19) and Korn's inequality. Next, setting

$$
\Upsilon(t):=a_{0}(u(t), u(t))+\|\nabla \mu(t)\|_{2}^{2}+\|\mathcal{L}(\psi(t))\|_{2, \Gamma}^{2},
$$

for every $t \geq 0$, from (6.18), we have $\Upsilon \in L^{1}(0, \infty)$. Let $\left(\phi_{*}, \psi_{*}\right)$ be an element of the $\omega$-limit set of $(\phi, \psi)$. By Lemma 6.2 with $\theta \in(0,1 / 2)$ (along with Remark 6.1)) and (6.22), we deduce that

$$
\begin{aligned}
\int_{t}^{\infty} \Upsilon(s) d s & \leq E_{\text {tot }}(t)-e_{*} \\
& =\widehat{\mathcal{E}}(\phi(t), \psi(t))-\widehat{\mathcal{E}}\left(\phi_{*}, \psi_{*}\right)+\frac{1}{2}|u(t)|^{2} \\
& \leq C|\Upsilon(t)|^{\frac{1}{2(1-\theta)}},
\end{aligned}
$$

for all $t>0$ such that $(\phi(t), \psi(t)) \in V_{\gamma}^{1}$ and $\left\|(\phi(t), \psi(t))-\left(\phi_{*}, \psi_{*}\right)\right\|_{V_{\gamma}^{0}} \leq \eta$. If we denote by $\Sigma$ this set, we infer by [25, Lemma 7.1] that $\Upsilon^{1 / 2} \in L^{1}(\Sigma)$. It follows that $u \in L^{1}\left(\Sigma ; \mathbb{H}^{1}\right)$, $\nabla \mu \in L^{1}\left(\Sigma ; L^{2}(\Omega)^{N}\right)$ and $\mathcal{L}(\psi) \in L^{1}\left(\Sigma ; L^{2}(\Gamma)\right)$. Therefore, we obtain exactly as in $(6.21)$ that $\left(B_{1}, B_{\Gamma}\right) \in L^{1}\left(\Sigma ;\left(V_{\gamma}^{1}\right)^{*}\right)$. This yields, according to $(2.7)-(2.8)$, that

$$
\left(\partial_{t} \phi, \partial_{t} \psi\right) \in L^{1}\left(\Sigma ;\left(V_{\gamma}^{1}\right)^{*}\right) \text {. }
$$

A simple contradiction argument (see [25]) shows that $\left(\partial_{t} \phi, \partial_{t} \psi\right)$ is absolutely integrable on $\left[\delta_{*}, \infty\right)$, for some $\delta_{*}>0$; henceforth the limit of $(\phi(t), \psi(t))$ exists as time goes to infinity. This implies that

$$
(\phi(t), \psi(t)) \rightarrow\left(\phi_{*}, \psi_{*}\right) \text { strongly in } V_{\gamma}^{0},
$$

as desired, owing to the precompactness of the whole trajectory $(\phi, \psi)$ in $V_{\gamma}^{0}$. The above convergence also holds strongly in $V_{\gamma}^{1-}=V_{\gamma}^{1-\eta}$, for some $\eta \in(0,1)$, on account of interpolation inequalities

$$
\|\phi\|_{H^{1-\eta}(\Omega)} \leq C\|\phi\|_{H^{1}(\Omega)}^{1-\eta}\|\phi\|_{L^{2}(\Omega)}^{\eta},\|\psi\|_{H^{1-\eta}(\Gamma)} \leq C\|\psi\|_{H^{1}(\Gamma)}^{1-\eta}\|\psi\|_{L^{2}(\Gamma)}^{\eta}
$$

and the uniform bounds in the second of (6.19). The proof of Theorem 3.3 is complete.

Remark 6.2. The convergence rate (3.12) is an immediate consequence of (3.8), (6.23) and the definition of $\Upsilon$. We omit the details.

Finally, we can demonstrate a conditional result which shows that a stronger convergence for $u$ may hold (cf. Theorem 3.4).

Proof of Theorem 3.4. Consider a sufficiently smooth sequence of solutions $\left(u_{\epsilon}, \phi_{\epsilon}, \psi_{\epsilon}\right)$ that satisfies conditions (i)-(ii) and the corresponding energy inequality (3.8) uniformly in $\epsilon>0$. Let us set $y_{\epsilon}(t):=\left|u_{\epsilon}(t)\right|^{2}$ and recall that $y_{\epsilon} \in L^{1}(0, \infty)$, uniformly in $\epsilon>0$, owing to the embedding $\mathbb{H}^{1} \hookrightarrow \mathbb{H}$ and (6.18). Our goal is to show that $y_{\epsilon} \in L^{\infty}(0, \infty)$ (cf. (3.6)) satisfies

$$
\frac{d y_{\epsilon}}{d t}(t) \leq \Theta_{\epsilon}(t), \text { for all } t \geq 0
$$

uniformly in $\epsilon>0$, for some function $\Theta_{\epsilon} \in L^{1}(0, \infty)$. Then, it follows that $y_{\epsilon}(t) \rightarrow 0$ as $t \rightarrow \infty$, uniformly in $\epsilon>0$, by application of [77, Lemma 6.2.1]. Therefore, passing to the limit as $\epsilon \rightarrow 0^{+}$ gives the claim for the weak solution $(u, \phi, \psi)$.

From equation (2.6), we have

$$
\begin{aligned}
\frac{1}{2} \frac{d y_{\epsilon}}{d t}(t)+a_{0}\left(u_{\epsilon}(t), u_{\epsilon}(t)\right) & =b_{1}\left(u_{\epsilon}(t), \phi_{\epsilon}(t), \mu_{\epsilon}(t)-\left\langle\mu_{\epsilon}(t)\right\rangle\right) \\
& +\left(\mathcal{L}\left(\psi_{\epsilon}(t)\right) \nabla_{\tau} \psi_{\epsilon}(t), u_{\epsilon \tau}(t)\right)_{\Gamma},
\end{aligned}
$$

for all $t \geq 0$. Observe that $b_{1}\left(u_{\epsilon}, \phi_{\epsilon}, \mu_{\epsilon}-\left\langle\mu_{\epsilon}\right\rangle\right)=\left\langle\bar{B}_{1}\left(\mu_{\epsilon}-\left\langle\mu_{\epsilon}\right\rangle, \phi_{\epsilon}\right), u_{\epsilon}\right\rangle$. Moreover, the form $\bar{B}_{1}: H^{1}(\Omega) \times\left(L^{2}(\Omega)\right)^{N} \rightarrow\left(\mathbb{H}^{1}\right)^{*}$ is bounded if $N \leq 3$. Thus, by the Korn and Poincaré's inequalities, we get

$$
b_{1}\left(u_{\epsilon}, \phi_{\epsilon}, \mu_{\epsilon}-\left\langle\mu_{\epsilon}\right\rangle\right) \leq\left\|u_{\epsilon}\right\|\left\|\bar{B}_{1}\left(\mu_{\epsilon}-\left\langle\mu_{\epsilon}\right\rangle, \phi_{\epsilon}\right)\right\|_{\left(\mathbb{H}^{1}\right)^{*}}
$$




$$
\leq \delta a_{0}\left(u_{\epsilon}, u_{\epsilon}\right)+\frac{C}{\delta}\left\|\nabla \mu_{\epsilon}\right\|_{2}^{2}\left\|\phi_{\epsilon}\right\|_{H^{1}}^{2}
$$

for any $\delta>0$. Concerning the term in (6.26), by trace theory we observe that

$$
\begin{aligned}
\left|\left(\mathcal{L}\left(\psi_{\epsilon}\right) \nabla_{\tau} \psi_{\epsilon}, u_{\epsilon \tau}\right)_{\Gamma}\right| & \leq\left\|\mathcal{L}\left(\psi_{\epsilon}\right) \nabla_{\tau} \psi_{\epsilon}\right\|_{\left(\mathbb{H}^{1 / 2}(\Gamma)\right)^{*}}\left\|u_{\epsilon}\right\| \\
& \leq \delta a_{0}\left(u_{\epsilon}, u_{\epsilon}\right)+\frac{C}{\delta}\left\|\mathcal{L}\left(\psi_{\epsilon}\right)\right\|_{2, \Gamma}^{2}\left\|\psi_{\epsilon}\right\|_{H^{1+s}(\Gamma)}^{2},
\end{aligned}
$$

for any $\delta>0$ and $C>0$ independent of time and $\epsilon>0$, provided that each term $\mathcal{L}\left(\psi_{\epsilon}\right) \partial_{\tau_{i}} \psi_{\epsilon}$ $(1 \leq i \leq N-1)$ belongs to $\left(H^{1 / 2}(\Gamma)\right)^{*}$ as a product of functions in $L^{2}(\Gamma)$ and $H^{s}(\Gamma)$, respectively. By Lemma 4.8, this is always the case if and only $s \geq 0$ satisfies $s>N / 2-1$. Collect now estimates (6.27)-(6.28) and insert them into the right-hand side of (6.26). Choosing a sufficiently small $\delta<1 / 2$, then taking into account the uniform in-time (and in $\epsilon>0$ ) bounds implied by (6.19), which also hold for $\left(u_{\epsilon}, \phi_{\epsilon}, \psi_{\epsilon}\right)$, and assumption (3.13), we readily infer the validity of (6.25) with

$$
\Theta_{\epsilon}:=C \delta^{-1}\left(\left\|\nabla \mu_{\epsilon}\right\|_{2}^{2}\left\|\phi_{\epsilon}\right\|_{H^{1}}^{2}+\left\|\mathcal{L}\left(\psi_{\epsilon}\right)\right\|_{2, \Gamma}^{2}\left\|\psi_{\epsilon}\right\|_{H^{1+s}(\Gamma)}^{2}\right) \in L^{1}(0, \infty) .
$$

The proof is finished.

\section{The Singular Potential CASE}

Proof of Theorem 3.5. The existence follows essentially from the statement of Theorem 3.2 and Remark 5.3. Indeed, we can construct smooth versions of $g_{\epsilon}, f_{1 \epsilon} \in C^{2}(\mathbb{R})$, obeying (5.1)-(5.2), and take a smooth monotone sequence $f_{0 \epsilon} \in C^{2}(\mathbb{R})$, approximating the singular part of the potential on compact subintervals of $(-1,1)$, still satisfying $(5.1)-(5.2)$, as well as $f_{0 \epsilon}(0)=0$ (see Step 1 of the proof of Theorem 3.2). In particular, we also have

$$
\left|f_{0 \epsilon}(s)\right| \leq\left|f_{0}(s)\right|,\left|F_{0 \epsilon}(s)\right| \leq\left|F_{0}(s)\right| \text {, for any } s \in(-1,1),
$$

and

$$
\lim _{\epsilon \rightarrow 0^{+}} f_{0 \epsilon}(s)=f_{0}(s), \lim _{\epsilon \rightarrow 0^{+}} F_{0 \epsilon}(s)=F_{0}(s), s \in(-1,1) .
$$

Hence the new "approximations" $f_{\epsilon}, g_{\epsilon}$ satisfy the conditions of Theorem 3.2. In addition, we may add an "artificial" viscosity term $\sigma \partial_{t} \phi$ in the equation for the chemical potential $\mu$, see (2.9). Let now $u_{\epsilon, \sigma}(0)=u_{0}, \phi_{\epsilon, \sigma}(0)=\phi_{0}, \psi_{\epsilon, \sigma}(0)=\psi_{0}$ with $\left(u_{0}, \phi_{0}, \psi_{0}\right) \in \mathbb{H} \times V_{\gamma}^{1}$ such that $F_{0}\left(\phi_{0}\right) \in L^{1}(\Omega)$ and $F_{0}\left(\psi_{0}\right) \in L^{1}(\Gamma)$. In this case, the existence of a sequence of solutions $\left(u_{\epsilon, \sigma}, \phi_{\epsilon, \sigma}, \psi_{\epsilon, \sigma}\right)$ in the sense of Definition 2.1 to the corresponding approximate problem follows from Remark 5.3; also, this sequence satisfies the (strong) energy inequality (5.88). Consequently, the estimates (5.56)-(5.57) are once again satisfied uniformly in $(\sigma, \epsilon) \in(0,1]^{2}$, as well as

$$
F_{\epsilon}\left(\phi_{\epsilon, \sigma}\right)=F_{0 \epsilon}\left(\phi_{\epsilon, \sigma}\right)+F_{1 \epsilon}\left(\phi_{\epsilon, \sigma}\right) \in L^{\infty}\left(0, T ; L^{1}(\Omega)\right)
$$

uniformly in $(\epsilon, \sigma)$, on account of the fact that $G_{\epsilon}(s) \geq-C_{G}$, uniformly for all $s \in \mathbb{R}$ (see (3.1)), $F_{1}(s)$ has at most quadratic growth as $|s| \rightarrow \infty$ and $\bar{F}_{0 \epsilon}(s)=\int_{0}^{s} f_{0 \epsilon}(\zeta) d \zeta$ is a convex potential on $\mathbb{R}$, satisfying (7.1)-(7.2).

We require additional uniform estimates with respect to $(\epsilon, \sigma)$. In what follows, we will exploit the following identity

$$
\begin{aligned}
& \left(\mathcal{L}\left(\psi_{\epsilon, \sigma}\right), \varphi\right)_{\Gamma}+\left(\mu_{\epsilon, \sigma}, \varphi\right) \\
& =\left(\nabla \phi_{\epsilon, \sigma}, \nabla \varphi\right)+\gamma\left(\nabla_{\tau} \psi_{\epsilon, \sigma}, \nabla_{\tau} \varphi\right)_{\Gamma}+\left(g\left(\psi_{\epsilon, \sigma}\right)+\zeta \psi_{\epsilon, \sigma}, \varphi\right)_{\Gamma} \\
& +\left(f_{0 \epsilon}\left(\phi_{\epsilon, \sigma}\right), \varphi\right)+\left(f_{1 \epsilon}\left(\phi_{\epsilon, \sigma}\right), \varphi\right)+\sigma\left(\partial_{t} \phi_{\epsilon, \sigma}, \varphi\right),
\end{aligned}
$$

which holds for all $\left(\varphi, \varphi_{\mid \Gamma}\right) \in V_{\gamma}^{1}$ (see (5.6)-(5.7)).

Step 1 (The $L_{t}^{2} H_{x}^{1}$-bound for $\left.\mu_{\epsilon, \sigma}\right)$. Let $\left\langle\phi_{0}\right\rangle=m \in(-1,1)$, where $\left(\phi_{0}, \psi_{0}\right) \in V_{\gamma}^{1}$, and recall that $\left\langle\phi_{\epsilon, \sigma}(t)\right\rangle=\left\langle\phi_{0}\right\rangle$, for all $t \geq 0$. Testing equation (7.3) with $\varphi=|\Omega|^{-1}$, we deduce

$$
\left\langle\mu_{\epsilon, \sigma}\right\rangle=\frac{|\Gamma|}{|\Omega|}\left\langle\widetilde{g}\left(\psi_{\epsilon, \sigma}\right)\right\rangle_{\Gamma}+\left\langle f_{\epsilon}\left(\phi_{\epsilon, \sigma}\right)\right\rangle-\frac{|\Gamma|}{|\Omega|}\left\langle\mathcal{L}\left(\psi_{\epsilon, \sigma}\right)\right\rangle_{\Gamma},
$$

where we have set $\langle\cdot\rangle_{\Gamma}=|\Gamma|^{-1} \int_{\Gamma}(\cdot) d S$ and recall that $f_{\epsilon}=f_{0 \epsilon}+f_{1 \epsilon}, \widetilde{g}(s)=g(s)+\zeta s$. Since $\nabla \mu_{\epsilon, \sigma} \in L^{2}\left(0, T ; L^{2}(\Omega)^{N}\right)$, uniformly by $(5.56)$, in order to prove that $\mu_{\epsilon, \sigma} \in L^{2}\left(0, T ; H^{1}(\Omega)\right)$, it suffices to show that $f_{0 \epsilon} \in L^{2}\left(0, T ; L^{1}(\Omega)\right)$, uniformly in $(\epsilon, \sigma)$ (indeed, the later bound is already 
valid for the smooth part $f_{1 \epsilon}$ of the potential $f_{\epsilon}$, owing to $(5.56)$ and $\left.f_{1}^{\prime} \in L^{\infty}(\mathbb{R})\right)$. We proceed as follows. We test the equation (5.4) with $\varphi=B_{N}^{-1}\left(\partial_{t} \phi_{\epsilon, \sigma}\right) \in H^{1}(\Omega)$ to deduce the identity

$$
\begin{aligned}
& \left\|\partial_{t} \phi_{\epsilon, \sigma}\right\|_{\left(H^{1}\right)^{*}}^{2}+\left(B_{N}^{1 / 2}\left(\mu_{\epsilon, \sigma}-\left\langle\mu_{\epsilon, \sigma}\right\rangle\right), B_{N}^{-1 / 2}\left(\partial_{t} \phi_{\epsilon, \sigma}\right)\right) \\
& =-\left(B_{1}\left(u_{\epsilon, \sigma}, \phi_{\epsilon, \sigma}\right), B_{N}^{-1} \partial_{t} \phi_{\epsilon, \sigma}\right) .
\end{aligned}
$$

Then a standard energy estimate, owing to Hölder and Young inequalities, allows one to derive

$$
\left\|\partial_{t} \phi_{\epsilon, \sigma}\right\|_{\left(H^{1}\right)^{*}}^{2} \leq \eta\left\|\partial_{t} \phi_{\epsilon, \sigma}\right\|_{\left(H^{1}\right)^{*}}^{2}+C_{\eta}\left(\left\|\nabla \mu_{\epsilon, \sigma}\right\|_{2}^{2}+\left\|B_{1}\left(u_{\epsilon, \sigma}, \phi_{\epsilon, \sigma}\right)\right\|_{H^{-1}}^{2}\right)
$$

for every $\eta>0$. By virtue of the uniform estimate for $\left(u_{\epsilon, \sigma}, \phi_{\epsilon, \sigma}\right)$, from (5.56) it is easy to show that $B_{1}$ is a bounded form from $\mathbb{H}^{1} \times L^{2}(\Omega)$ into $\left(H^{1}(\Omega)\right)^{*}$; henceforth, it follows that

$$
\partial_{t} \phi_{\epsilon, \sigma} \in L^{2}\left(0, T ;\left(H^{1}(\Omega)\right)^{*}\right) \text { uniformly in }(\epsilon, \sigma),
$$

exactly as in (5.63). Next, we can test (7.3) by $\bar{\phi}_{\epsilon, \sigma}=\phi_{\epsilon, \sigma}-m$ to obtain

$$
\begin{aligned}
& \left(\mathcal{L}\left(\psi_{\epsilon, \sigma}\right), \psi_{\epsilon, \sigma}-m\right)_{\Gamma}+\left(\mu_{\epsilon, \sigma}-\left\langle\mu_{\epsilon, \sigma}\right\rangle, \bar{\phi}_{\epsilon, \sigma}\right) \\
& =\left\|\left(\bar{\phi}_{\epsilon, \sigma}, \bar{\psi}_{\epsilon, \sigma}\right)\right\|_{V_{\gamma}^{1}}^{2}+\zeta m\left(1, \bar{\psi}_{\epsilon, \sigma}\right)_{\Gamma}+\left(f_{0 \epsilon}\left(\phi_{\epsilon, \sigma}\right), \bar{\phi}_{\epsilon, \sigma}\right) \\
& +\left(f_{1 \epsilon}\left(\phi_{\epsilon, \sigma}\right), \bar{\phi}_{\epsilon, \sigma}\right)+\left(g\left(\psi_{\epsilon, \sigma}\right), \bar{\psi}_{\epsilon, \sigma}\right)_{\Gamma}+\sigma\left(\partial_{t} \phi_{\epsilon, \sigma}, \bar{\phi}_{\epsilon, \sigma}\right) .
\end{aligned}
$$

Owing now to the inequalities

$$
\left\{\begin{array}{l}
f_{0 \epsilon}(s)(s-m) \geq \alpha\left|F_{0 \epsilon}(s)\right|-C_{f}, \\
f_{0 \epsilon}(s)(s-m) \geq \alpha\left|f_{0 \epsilon}(s)\right|-C_{f}
\end{array}\right.
$$

which hold for all $s \in \mathbb{R}$, for some positive constants $\alpha, C_{f}>0$ independent of $(\epsilon, \sigma) \in(0,1]^{2}$ (see, e.g., $[34,35,53])$, and the uniform estimates (5.56), (7.5), we easily arrive at the uniform bound $f_{0 \epsilon} \in L^{1}\left(0, T ; L^{1}(\Omega)\right)$. We note that, on the basis of $(7.4)$, we also find that $\left\langle\mu_{\epsilon, \sigma}\right\rangle \in L^{1}(0, T)$ uniformly. Furthermore, on account of assumptions (3.1)-(3.2) for $f_{1}$ and $g$, we deduce from (7.6) a bound of the form

$$
\begin{aligned}
& \left\|\left(\bar{\phi}_{\epsilon, \sigma}, \bar{\psi}_{\epsilon, \sigma}\right)\right\|_{V_{\gamma}^{1}}^{2}+\alpha\left\|f_{0 \epsilon}\left(\phi_{\epsilon, \sigma}\right)\right\|_{1} \\
& \leq C+\sigma\left\|\partial_{t} \phi_{\epsilon, \sigma}\right\|_{\left(H^{1}\right)^{*}}\left\|\phi_{\epsilon, \sigma}\right\|_{H^{1}(\Omega)}+C\left\|\nabla \mu_{\epsilon, \sigma}\right\|_{2}\left\|\bar{\phi}_{\epsilon, \sigma}\right\|_{2} \\
& +C\left\|\mathcal{L}\left(\psi_{\epsilon, \sigma}\right)\right\|_{2, \Gamma}\left\|\bar{\psi}_{\epsilon, \sigma}\right\|_{2, \Gamma},
\end{aligned}
$$

for some constant $C>0$ indepedent of $(\epsilon, \sigma)$. Squaring both sides of this inequality and integrating over $(0, T)$, we immediately deduce that

$$
f_{0 \epsilon}\left(\phi_{\epsilon, \sigma}\right) \in L^{2}\left(0, T ; L^{1}(\Omega)\right) \text { uniformly in }(\epsilon, \sigma) .
$$

This allows to improve the regularity of $\mu_{\epsilon, \sigma}$ to $\left\langle\mu_{\epsilon, \sigma}\right\rangle \in L^{2}(0, T)$, exploiting (7.4) and estimates (5.56) once again. Finally, by the Poincaré inequality we conclude that

$$
\mu_{\epsilon, \sigma} \in L^{2}\left(0, T ; H^{1}(\Omega)\right) \text { uniformly in }(\epsilon, \sigma),
$$

exactly as in the regular potential case (see (5.58)).

Step 2 (The $L_{t}^{2} L_{x}^{2}$-bound for $\left.f_{0 \epsilon}\right)$. This time we take $\varphi=f_{0 \epsilon}\left(\phi_{\epsilon, \sigma}\right)$ in $(7.3)$, which is clearly an admissible test function on account of the regularity of $f_{0 \epsilon}$ and $\phi_{\epsilon, \sigma}$. This multiplication yields

$$
\begin{aligned}
& \left(\mathcal{L}\left(\psi_{\epsilon, \sigma}\right), f_{0 \epsilon}\left(\psi_{\epsilon, \sigma}\right)\right)_{\Gamma}+\left(\mu_{\epsilon, \sigma}, f_{0 \epsilon}\left(\phi_{\epsilon, \sigma}\right)\right) \\
& =\left(f_{0 \epsilon}^{\prime}\left(\phi_{\epsilon, \sigma}\right) \nabla \phi_{\epsilon, \sigma}, \nabla \phi_{\epsilon, \sigma}\right)+\gamma\left(f_{0 \epsilon}^{\prime}\left(\psi_{\epsilon, \sigma}\right) \nabla_{\tau} \psi_{\epsilon, \sigma}, \nabla_{\tau} \psi_{\epsilon, \sigma}\right)_{\Gamma} \\
& +\left\|f_{0 \epsilon}\left(\phi_{\epsilon, \sigma}\right)\right\|_{2}^{2}+\left(f_{1 \epsilon}\left(\phi_{\epsilon, \sigma}\right), f_{0 \epsilon}\left(\phi_{\epsilon, \sigma}\right)\right)+\left(\widetilde{g}\left(\psi_{\epsilon, \sigma}\right), f_{0 \epsilon}\left(\psi_{\epsilon, \sigma}\right)\right)_{\Gamma} \\
& +\sigma\left(\partial_{t} \phi_{\epsilon, \sigma}, f_{0 \epsilon}\left(\phi_{\epsilon, \sigma}\right)\right) .
\end{aligned}
$$

Furthermore, we shall test with $\eta=f_{0}\left(\psi_{\epsilon, \sigma}\right) \in L^{2}(\Gamma)$ in the identity

$$
\left(\partial_{t} \psi_{\epsilon, \sigma}, \eta\right)_{\Gamma}+\left(B_{\Gamma}\left(u_{\epsilon, \sigma}, \psi_{\epsilon, \sigma}\right), \eta\right)+\left(\mathcal{L}_{\epsilon}\left(\psi_{\epsilon, \sigma}\right), \eta\right)_{\Gamma}=0
$$

in order to get

$$
\left(\mathcal{L}\left(\psi_{\epsilon, \sigma}\right), f_{0 \epsilon}\left(\psi_{\epsilon, \sigma}\right)\right)_{\Gamma}=-\frac{d}{d t}\left(F_{0 \epsilon}\left(\psi_{\epsilon, \sigma}\right), 1\right)_{\Gamma}-\int_{\Gamma}\left(u_{\tau}\right)_{\epsilon, \sigma} \cdot \nabla_{\tau} \psi_{\epsilon, \sigma} f_{0}\left(\psi_{\epsilon, \sigma}\right) d S .
$$


Since $f_{0 \epsilon}^{\prime}(s) \geq 0$ (and so $\left.f_{0 \epsilon}(s) s \geq 0\right)$, from (7.10)-(7.11) we deduce

$$
\begin{aligned}
& \frac{d}{d t}\left(F_{0 \epsilon}\left(\psi_{\epsilon, \sigma}\right), 1\right)_{\Gamma}+\left\|f_{0 \epsilon}\left(\phi_{\epsilon, \sigma}\right)\right\|_{2}^{2}+\left(f_{0 \epsilon}^{\prime}\left(\phi_{\epsilon, \sigma}\right) \nabla \phi_{\epsilon, \sigma}, \nabla \phi_{\epsilon, \sigma}\right) \\
& +\gamma\left(f_{0 \epsilon}^{\prime}\left(\psi_{\epsilon, \sigma}\right) \nabla_{\tau} \psi_{\epsilon, \sigma}, \nabla_{\tau} \psi_{\epsilon, \sigma}\right)_{\Gamma} \\
& +\left(g\left(\psi_{\epsilon, \sigma}\right), f_{0 \epsilon}\left(\psi_{\epsilon, \sigma}\right)\right)_{\Gamma} \\
& =\left(\mu_{\epsilon, \sigma}, f_{0 \epsilon}\left(\phi_{\epsilon, \sigma}\right)\right)-\left(f_{1 \epsilon}\left(\phi_{\epsilon, \sigma}\right), f_{0 \epsilon}\left(\phi_{\epsilon, \sigma}\right)\right)-\sigma\left(\partial_{t} \phi_{\epsilon, \sigma}, f_{0 \epsilon}\left(\phi_{\epsilon, \sigma}\right)\right) \\
& -\int_{\Gamma}\left(u_{\tau}\right)_{\epsilon, \sigma} \cdot \nabla_{\tau} \psi_{\epsilon, \sigma} f_{0}\left(\psi_{\epsilon, \sigma}\right) d S .
\end{aligned}
$$

The first three terms on the right-hand side of (7.12) can be estimated by Young's inequality, in terms of

$$
\eta\left\|f_{0 \epsilon}\left(\phi_{\epsilon, \sigma}\right)\right\|_{2}^{2}+C_{\eta}\left(\sigma\left\|\partial_{t} \phi_{\epsilon, \sigma}\right\|_{2}^{2}+\left\|\mu_{\epsilon, \sigma}\right\|_{2}^{2}+\left\|f_{1 \epsilon}\left(\phi_{\epsilon, \sigma}\right)\right\|_{2}^{2}\right)
$$

for any $\eta>0$. For the last term, for every $\delta>0$ we deduce

$$
\begin{aligned}
& \left|\int_{\Gamma}\left(u_{\tau}\right)_{\epsilon, \sigma} \cdot \nabla_{\tau} \psi_{\epsilon, \sigma} f_{0}\left(\psi_{\epsilon, \sigma}\right) d S\right| \\
& \leq C_{\delta, \gamma}\left\|\left(u_{\tau}\right)_{\epsilon, \sigma}\right\|_{2, \Gamma}^{2}+\delta \gamma \int_{\Gamma}\left|\nabla_{\tau} \psi_{\epsilon, \sigma}\right|^{2}\left(f_{0}\left(\psi_{\epsilon, \sigma}\right)\right)^{2} d S .
\end{aligned}
$$

We further deduce from assumptions (3.16)-(3.17) that

$$
f_{0 \epsilon}(s) g_{\epsilon}(s) \geq-C_{M}, \text { for all } s \in \mathbb{R} \backslash[-M, M]
$$

as well as

$$
f_{0 \epsilon}^{\prime}(s)-\delta\left(f_{0 \epsilon}(s)\right)^{2} \geq-C_{\delta, M}, \text { for all } s \in \mathbb{R} \backslash[-M, M]
$$

with constants $C_{\delta, M}, C_{M}>0$ independent of $\epsilon \in\left(0, \epsilon_{0}\right]$, for some $\epsilon_{0}>0$ (These inequalities can be easily proven from (3.16)-(3.17) for the approximating families; we omit the details). Combining these estimates and choosing a sufficiently small $\eta \ll 1$, we obtain

$$
\begin{aligned}
\frac{d}{d t}\left(F_{0 \epsilon}\left(\psi_{\epsilon, \sigma}\right), 1\right)_{\Gamma}+ & \left\|f_{0 \epsilon}\left(\phi_{\epsilon, \sigma}\right)\right\|_{2}^{2}+\left(f_{0 \epsilon}^{\prime}\left(\phi_{\epsilon, \sigma}\right) \nabla \phi_{\epsilon, \sigma}, \nabla \phi_{\epsilon, \sigma}\right) \\
& \leq C_{\eta, M}\left(1+\left\|f_{1 \epsilon}\left(\phi_{\epsilon, \sigma}\right)\right\|_{2}^{2}+\left\|\mu_{\epsilon, \sigma}\right\|_{2}^{2}+\sigma\left\|\partial_{t} \phi_{\epsilon, \sigma}\right\|_{2}^{2}\right) \\
& +\gamma C_{\delta, M}\left\|\nabla_{\tau} \psi_{\epsilon, \sigma}\right\|_{2, \Gamma}^{2}+C_{\delta, \gamma}\left\|\left(u_{\tau}\right)_{\epsilon, \sigma}\right\|_{2, \Gamma}^{2} .
\end{aligned}
$$

We also note here that $\gamma>0$ is important for the estimate in (7.13) owing to

$$
\begin{aligned}
& \gamma\left(f_{0 \epsilon}^{\prime}\left(\psi_{\epsilon, \sigma}\right) \nabla_{\tau} \psi_{\epsilon, \sigma}, \nabla_{\tau} \psi_{\epsilon, \sigma}\right)_{\Gamma}-\delta \gamma \int_{\Gamma}\left|\nabla_{\tau} \psi_{\epsilon, \sigma}\right|^{2}\left(f_{0}\left(\psi_{\epsilon, \sigma}\right)\right)^{2} d S \\
& \geq-\gamma C_{\delta, M}\left\|\nabla_{\tau} \psi_{\epsilon, \sigma}\right\|_{2, \Gamma}^{2} .
\end{aligned}
$$

Since $\sigma \in(0,1]$, the uniform bounds of (5.56)-(5.57) together with (7.9) yield that

$$
\begin{gathered}
F_{0 \epsilon}\left(\psi_{\epsilon, \sigma}\right) \in L^{\infty}\left(0, T ; L^{1}(\Gamma)\right) \text { uniformly in }(\epsilon, \sigma), \\
f_{0 \epsilon}\left(\phi_{\epsilon, \sigma}\right) \in L^{2}\left(0, T ; L^{2}(\Omega)\right) \text { uniformly in }(\epsilon, \sigma),
\end{gathered}
$$

provided that $F_{0}\left(\psi_{0}\right) \in L^{1}(\Gamma)$ (indeed, recall (7.1)-(7.2)). This proves our claim.

Step 3 (Final argument). With the bound (7.16) at our disposal, we can now argue verbatim by elliptic regularity as in (5.59) to deduce the uniform bound $\left(\phi_{\epsilon, \sigma}, \psi_{\epsilon, \sigma}\right) \in L^{2}\left(0, T ; V_{\gamma}^{2}\right)$. This bound is also sufficient to pass to the limit strongly in the sequence $\left(u_{\epsilon, \sigma}, \phi_{\epsilon, \sigma}, \psi_{\epsilon, \sigma}\right)$ exactly as in the proof of Theorem 3.2 (see Step 3, (5.74)-(5.76)). The only (minor) difference is now in (5.77), where for the sequence $f_{\epsilon}=f_{0 \epsilon}+f_{1 \epsilon}$ we have instead

$$
\left\{\begin{array}{l}
f_{1 \epsilon}\left(\phi_{\epsilon, \sigma}\right) \rightarrow f_{1}(\phi) \text { strongly in } L^{2}\left(0, T ; L^{2}(\Omega)\right), \\
f_{0 \epsilon}\left(\phi_{\epsilon, \sigma}\right) \rightarrow \xi^{*} \text { strongly in } L^{2}\left(0, T ; L^{2}(\Omega)\right),
\end{array}\right.
$$

as $(\epsilon, \sigma) \rightarrow(0,0)$. The identification $\xi^{*}=f_{0}(\phi)$ a.e. in $\Omega \times(0, T)$ can be easily proven by a standard monotonicity argument, exploiting the identity (7.3) and the convergence properties (5.75), (5.76), and the last three consequences of (5.77). Finally, every limit point $(u, \phi, \psi)$ of the sequence of solutions $\left(u_{\epsilon, \sigma}, \phi_{\epsilon, \sigma}, \psi_{\epsilon, \sigma}\right)$ satisfies the energy inequality (3.6), by application of Fatou's lemma exactly as in (5.86) (indeed, both $F_{0}$ and $F_{0 \epsilon}$ are convex functions on $(-1,1)$ and $\mathbb{R}$, respectively). We also emphasize that any energy weak solution $(u, \phi, \psi)$, obtained from the 
approximation scheme that we exploited in the previous sections, also satisfies the stronger energy inequality (3.8), owing to the proof of Proposition 6.3 and the fact that the convex potential $F_{0}$ satisfies (7.1)-(7.2). The proof of this fact requires only minor inessential modifications. This concludes the proof of Theorem 3.5.

As in Lemma 6.1 in the regular potential case, we can prove that every energy solution given by Definition 3.1 has a non-empty $\omega$-limit set.

Lemma 7.1. Le the assumptions of Theorem 3.5 be satisfied and suppose that $h \equiv 0$. Let $(u, \phi, \psi)$ be an energy solution in the sense of Definition 3.1. Then the $\omega$-limit set of $(u, \phi, \psi)$ is nonempty and the same conclusion of Lemma 6.1 holds as well. In addition, each solution $\left(\phi_{*}, \psi_{*}\right)$ of the elliptic boundary value problem (6.3) belongs to $V_{\gamma}^{2} \cap\left\{\phi_{*}:\left\langle\phi_{*}\right\rangle=\left\langle\phi_{0}\right\rangle \in(-1,1)\right\}$, with $f_{0}\left(\phi_{*}\right) \in L^{2}(\Omega)$. Finally, there exists a constant $\varsigma \in(0,1)$, depending only on $f, g, \bar{\Omega}$ and $\left\langle\phi_{0}\right\rangle=m \in(-1,1)$, such that

$$
-1+\varsigma \leq \phi_{*}(x) \leq 1-\varsigma, \text { for all } x \in \bar{\Omega} .
$$

Proof. The first part of the lemma, which states that the $\omega$-limit set of $(u, \phi, \psi)$ is nonempty, follows in the same fashion as in the proof of Lemma 6.1 without any modifications. The existence of at least one strong solution to problem (6.3), i.e., $d_{*}$ is constant, and

$$
-\Delta \phi_{*}+f\left(\phi_{*}\right)=d_{*}, \text { in } \Omega, \quad-\gamma A_{\tau} \psi_{*}+\partial_{n} \phi_{*}+\zeta \psi_{*}+g\left(\psi_{*}\right)=0, \text { on } \Gamma,
$$

follows immediately from the balance condition (3.16) between the functions $f_{0}, g$ and the proof of Theorem 3.5 (see, in particular, Steps 1-3). Moreover, we also obtain the estimate

$$
\left\|\left(\phi_{*}, \psi_{*}\right)\right\|_{V_{\gamma}^{2}}+\left\|f_{0}\left(\phi_{*}\right)\right\|_{2} \leq C_{m},
$$

for some constant $C_{m}>0$. By the embedding $H^{2}(\Omega) \hookrightarrow C^{0, \kappa}(\bar{\Omega})$ if $\mathrm{N} \leq 3$, it clearly holds $\phi_{*} \in C^{0, \kappa}(\bar{\Omega})$. It is also worth observing that the previous estimate implies that the constant in (7.18) can be controlled explicitly since

$$
|\Omega| d_{*}=\int_{\Omega}\left(f_{0}\left(\phi_{*}\right)+f_{1}\left(\phi_{*}\right)\right) d x+\int_{\Gamma}\left(\zeta \psi_{*}+g\left(\psi_{*}\right)\right) d x .
$$

In particular, on account of (7.19) one also has $\left|d_{*}\right| \leq C_{m}^{\prime}$, for some $C_{m}^{\prime}>0$ that depends on $C_{m}$. Moreover, we have $-1<\phi_{*}<1$ almost everywhere in $\Omega$, owing to $f_{0}\left(\phi_{*}\right) \in L^{2}(\Omega)$ and $(3.15)$.

It remains to show the strict separation property $(7.17)$. First we note that due to assumption (3.15) we can find a sufficiently small $\varsigma \in(0,1)$, depending only on $f, m$ and the measure of $\bar{\Omega}$ such that

$$
f_{0}(s)+f_{1}(s)-d_{*} \geq 0 \text { on }[1-\varsigma, 1) \text {, and } f_{0}(s)+f_{1}(s)-d_{*} \leq 0 \text { on }(-1,-1+\varsigma] .
$$

On the other hand, due to the fact that $g \in C([-1,1])$ and from $(3.16)$ it also holds

$$
g(s) \geq 0 \text { on }[1-\varsigma, 1) \text { and } g(s) \leq 0 \text { on }(-1,-1+\varsigma]
$$

see Remark 3.4. Next, define $z=\left(\phi_{*}-1+\varsigma\right)_{+}$and observe that $\left(z, z_{\mid \Gamma}\right) \in V_{\gamma}^{1}$ with $\nabla \phi_{*} \equiv \nabla z$ on $\Omega \cap\{z>0\}, \nabla_{\tau} \psi_{*} \equiv \nabla_{\tau} z$ on $\Gamma \cap\{z>0\}$ and $\nabla z=\nabla_{\tau} z \equiv 0$ on the sets $\{x \in \bar{\Omega}: z=0\}$. To this end, multiply the first and second equations of (7.18) by $\phi_{*}$, then integrate the corresponding relations over $\Omega$ and $\Gamma$, respectively. We deduce that

$$
\|\nabla z\|_{2}^{2}+\gamma\left\|\nabla_{\tau} z\right\|_{2, \Gamma}^{2}+\zeta \int_{\Gamma} \psi_{*} z d S+\int_{\Omega}\left(f\left(\phi_{*}\right)-d_{*}\right) z d x+\int_{\Gamma} g\left(\psi_{*}\right) z d S=0 .
$$

It follows from (7.20)-(7.21) and the definition of $z$ that

$$
\int_{\Omega}\left(f\left(\phi_{*}\right)-d_{*}\right) z d x+\int_{\Gamma} g\left(\psi_{*}\right) z d S \geq 0 .
$$

It is also easy to see that

$$
\int_{\Gamma} \psi_{*} z d S \geq \int_{\Gamma} z^{2} d S
$$

Thus, it follows from (7.22) that $z \equiv 0$ in the $V_{\gamma}^{1}$-norm, completing the claim. In order to prove the uniform separation property from the point $s=-1$ of the singular potential $f_{0}(s)$, one defines a new function $\tilde{z}=\left(-1+\varsigma-\phi_{*}\right)$ and argues in a similar fashion, owing to the same conclusions (7.20)-(7.21)). Lemma 7.1 is proved. 


\section{Appendix: Derivation of the MOdel}

In this section, we briefly recall the physical derivation of the model (1.1)-(1.10) introduced in $[58,59]$. To this end, we first consider the energy functional associated with the fluid motion

$$
\mathcal{F}_{N S}(u)=\int_{\Omega} \nu|D(u)|^{2} d x+\int_{\Gamma} \frac{\beta}{2}\left|u_{\tau}\right|^{2} d S
$$

Here $d S$ denotes the surface measure, while the integral over $\Gamma$ accounts for fluid wall slip. Concerning the free energy functional stabilizing the interface separating the two fluids, we postulate

$$
\mathcal{F}_{\text {tot }}(\phi)=\int_{\Omega}\left(\frac{\varepsilon}{2}|\nabla \phi|^{2}+\varepsilon^{-1} F(\phi)\right) d x+\int_{\Gamma}\left(\frac{\gamma}{2}\left|\nabla_{\tau} \phi\right|^{2}+\frac{\zeta}{2}|\phi|^{2}+G(\phi)\right) d S .
$$

Note that only the case $\gamma=0$ was considered in [59] (and any of its references). Here a boundary contribution has been added to $\mathcal{F}(\phi)$ (cf. (1.4)) to account for possible fluid-solid interactions taking place at the boundary. Based on the definitions of the functionals above, a hydrodynamic model for the contact line motion can be derived as follows. Let us observe now that the incompressible Navier-Stokes equations (1.1)-(1.2) is nothing but the momentum equation for the (average) velocity field $u$. The last term $\mu \nabla \phi$ in (1.1) accounts for stress forces at the fluidfluid interface [61], while equation (1.3) is a reformulation of mass conservation (see, for instance, [59]). In order to derive the boundary conditions (1.6)-(1.8), we also need to introduce a further dissipative functional

$$
\mathcal{F}_{C H}(\phi)=\int_{\Omega} \frac{\varrho_{0}}{2}|\nabla \mu|^{2} d x+\int_{\Gamma} \frac{1}{2 l_{0}}\left|\partial_{t}^{\tau} \phi\right|^{2} d S,
$$

in order to construct the total rate of energy dissipation, namely,

$$
\mathcal{F}_{\text {dis }}(u, \phi)=\mathcal{F}_{N S}(u)+\mathcal{F}_{C H}(\phi) \text {. }
$$

Observe that $\partial_{t}^{\tau} \phi($ see $(8.3))$ denotes the material time derivative of $\phi$ at the solid surface $\Gamma$, i.e.,

$$
\partial_{t}^{\tau} \phi=\partial_{t} \phi+u_{\tau} \cdot \nabla_{\tau} \phi
$$

Note that the right-hand side of equation (8.4) consists of four quadratic terms corresponding to the four physically distinct sources of dissipation: the shear viscosity in the bulk, the fluid wall slipping, the bulk diffusion and the wall relaxation.

The total free energy $\mathcal{F}_{\text {tot }}(\phi)$ rate change can be written as

$$
\frac{\partial}{\partial t} \mathcal{F}_{t o t}(\phi(t))=\int_{\Omega} \mu(t) \partial_{t} \phi(t) d x+\int_{\Gamma} \mathcal{L}(\phi(t)) \partial_{t} \phi(t) d S
$$

where $\mu$ is the chemical potential (1.6) and $\mathcal{L}$ is defined in (1.9). Using the Cahn-Hilliard equation (1.3) and the fact that $\partial_{n} \mu=0$ on $\Gamma \times(0, \infty)$, we obtain from $(8.5)$

$$
\frac{\partial}{\partial t} \mathcal{F}_{t o t}(\phi)=\int_{\Omega}\left(\varrho_{0}|\nabla \mu|^{2}-\mu u \cdot \nabla \phi\right) d x+\int_{\Gamma} \mathcal{L}(\phi)\left(\partial_{t}^{\tau} \phi-u_{\tau} \cdot \nabla_{\tau} \phi\right) d S
$$

On the other hand, the first law of thermodynamics requires

$$
\frac{\partial}{\partial t} \mathcal{F}_{t o t}(\phi)=-T \frac{\partial E}{\partial t}+\frac{\partial W}{\partial t}
$$

where $E$ and $W$ denote the entropy and work, respectively. Here $T$ denotes temperature which is assumed to be uniform in the fluid. Note that the entropy part $-T \partial S / \partial t$ must arise from the bulk diffusion and the wall relaxation, while the work rate $\partial W / \partial t$ is due to the work done by the flow at the fluid-fluid interface. More precisely, we have

$$
-T \frac{\partial E}{\partial t}=\int_{\Omega} \varrho_{0}|\nabla \mu|^{2} d x+\int_{\Gamma} \mathcal{L}(\phi) \partial_{t}^{\tau} \phi d S
$$

and

$$
\frac{\partial W}{\partial t}=-\int_{\Omega} u \cdot(\mu \nabla \phi) d x-\int_{\Gamma} u_{\tau} \cdot\left(\mathcal{L}(\phi) \nabla_{\tau} \phi\right) d S .
$$

We can see from (8.7) that $\mu \nabla \phi$ and $\mathcal{L}(\phi) \nabla_{\tau} \phi$ are forces (stresses) exerted by the interface on the flow. In order to derive the correct boundary conditions for two-phase flows, one may employ Onsager's variational principle of energy dissipation for incompressible single-phase flows 
(cf. [56, 57]; see also [59, (3.1)-(3.5)]). Therefore, a hydrodynamic model for the contact-line motion for two-phase flows can be derived by minimizing

$$
\mathcal{F}_{\text {dis }}(u, \phi)+\partial_{t} \mathcal{F}_{\text {tot }}(\phi)
$$

with respect to the rates $\left\{u, \nabla \mu, \partial_{t}^{\tau} \phi\right\}$ subject to the incompressibility condition (1.2) and (1.6)(1.9) (see, for instance, $[59,(3.28)-(3.32)]$ ). It is worth mentioning that an important point of this derivation is that the uncompensated Young stress at the boundary $\mathcal{L}(\phi) \nabla_{\tau} \phi$ must accompany, according to (8.7), the capillary force density in the bulk (i.e., the term $\mu \nabla \phi$ ), both being interfacial forces. Therefore, the term $\mathcal{L}(\phi) \nabla_{\tau} \phi$ is simply an indicator of the fluid-fluid interfacial tension at the solid boundary $\Gamma$.

Acknowledgement 8.1. The second author is a member of the Gruppo Nazionale per l'Analisi Matematica, la Probabilità e le loro Applicazioni (GNAMPA) of the Istituto Nazionale di Alta Matematica (INdAM). We also wish to acknowledge the reviewer whose comments and important remarks have improved the initial version of the article. We thank Hao Wu for pointing out a gap in a previous proof of Theorem 3.3.

\section{REFERENCES}

[1] H. Abels, On a diffuse interface model for two-phase flows of viscous, incompressible fluids with matched densities, Arch. Ration. Mech. Anal. 194 (2009), 463-506.

[2] H. Abels, Strong well-posedness of a diffuse interface model for a viscous, quasi-incompressible two-phase flow. SIAM J. Math. Anal. 44 (2012), 316-340.

[3] H. Abels, E. Feireisl, On a diffuse interface model for a two-phase flow of compressible viscous fluids, Indiana Univ. Math. J. 57 (2008), 659-698.

[4] H. Abels, M. Wilke, Convergence to equilibrium for the Cahn-Hilliard equation with a logarithmic free energy, Nonlinear Anal. 67 (2007), 3176-3193.

[5] D.M. Anderson, G.B. McFadden, A.A. Wheeler, Diffuse-interface methods in fluid mechanics, Annual review of fluid mechanics, Vol. 30, Annual Reviews, Palo Alto, CA, 139-165, 1998.

[6] V.E. Badalassi, H.D. Ceniceros, S. Banerjee, Computation of multiphase systems with phase field models, J. Comput. Phys. 190 (2003), 371-397.

[7] K. Bao, Y. Shi, S. Sun, X.-P. Wang, A finite element method for the numerical solution of the coupled Cahn-Hilliard and Navier-Stokes system for moving contact line problems, J. Comput. Phys. 231 (2012), 8083-8099.

[8] A. Berti, V. Berti, D. Grandi, Well-posedness of an isothermal diffusive model for binary mixtures of incompressible fluids, Nonlinearity 24 (2011), 3143-3164.

[9] F. Boyer, Mathematical study of multi-phase flow under shear through order parameter formulation, Asymp. Anal. 20 (1999), 175-212.

[10] F. Boyer, Nonhomogenous Cahn-Hilliard fluids, Ann. Inst. H. Poincaré Anal. Non Linéaire 18 (2001) $225-259$.

[11] F. Boyer, A theoretical and numerical model for the study of incompressible model flows, Computers \& Fluids 31 (2002), 41-68.

[12] F. Boyer, P. Fabrie, Persistency of 2D perturbations of one-dimensional solutions for a Cahn-Hilliard flow model under high shear, Asympt. Anal. 33 (2003), 107-151.

[13] T. Blesgen, A generalization of the Navier-Stokes equation to two-phase flows, J. Physics D (Applied Physics) 32 (1999), 1119-1123.

[14] S. Bosia, S. Gatti, Pullback exponential attractor for a Cahn-Hilliard-Navier-Stokes system in 2D, Dyn. Partial Differ. Equ. 11 (2014), 1-38.

[15] S. Bosia, M. Grasselli, A. Miranville, On the longtime behavior of a $2 D$ hydrodynamic model for chemically reacting binary fluid mixtures, Math. Methods Appl. Sci. 37 (2014), 726-743.

[16] C. Cao, C.G. Gal, Global solutions for the 2D NS-CH model for a two-phase flow of viscous, incompressible fluids with mixed partial viscosity and mobility, Nonlinearity 25 (2012), 3211-3234.

[17] C. Cavaterra, C.G. Gal, M. Grasselli, A. Miranville, Phase-field systems with nonlinear coupling and dynamic boundary conditions, Nonlinear Anal. 72 (2010), 2375-2399.

[18] R. Chella, J. Viñals, Mixing of a two-phase fluid by a cavity flow, Phys. Rev. E 53 (1996), 3832-3840.

[19] V.V. Chepyzhov, M.I. Vishik, Attractors for equations of mathematical physics, American Mathematical Society Colloquium Publications 49, American Mathematical Society, Providence, RI, 2002.

[20] L. Cherfils, A. Miranville, S. Zelik, The Cahn-Hilliard equation with logarithmic potentials, Milan J. Math. 79 (2011), 561-596.

[21] E.B. Dussan V, The moving contact line: the slip boundary condition, J. Fluid Mech. 77 (1976), 665-684.

[22] E.B. Dussan V, S.H. Davis, On the motion of a fluid-fluid interface along a solid surface, J. Fluid Mech. 65 (1974), 71-95.

[23] X. Feng, Fully discrete finite element approximation of the Navier-Stokes-Cahn-Hilliard diffuse interface model for two-phase flows, SIAM J. Numer. Anal. 44 (2006), 1049-1072.

[24] X. Feng, Y. He, C. Liu, Analysis of finite element approximations of a phase field model for two-phase fluids, Math. Comp. 76 (2007), 539-571.

[25] E. Feireisl, F. Simondon, Convergence for semilinear degenerate parabolic equations in several space dimensions, J. Dynam. Differ. Equ. 12 (2000), 647-673. 
[26] H.P. Fischer, P. Maass, W. Dieterich, Novel surface modes in spinodal decomposition, Phys. Rev. Letters 79 (1997), 893-896.

[27] H.P. Fischer, P. Maass, W. Dieterich, Diverging time and length scales of spinodal decomposition modes in thin flows, Europhys. Letters 42 (1998), 49-54.

[28] C.G. Gal, M. Grasselli, Asymptotic behavior of a Cahn-Hilliard-Navier-Stokes system in 2D, Ann. Inst. H. Poincaré Anal. Non Linéaire 27 (2010), 401-436.

[29] C.G. Gal, M. Grasselli, Instability of two-phase flows: a lower bound on the dimension of the global attractor of the Cahn-Hilliard-Navier-Stokes system, Phys. D 240 (2011), 629-635.

[30] C.G. Gal, M. Grasselli, Trajectory and global attractors for binary mixtures fluid flows in 3D, Chin. Ann. Math. Ser. B 31 (2010), 655-678.

[31] C.G. Gal, T. Medjo, On a regularized family of models for homogeneous incompressible two-phase flows, J. Nonlinear Sci. 24 (2014), 1033-1103.

[32] C.G. Gal, M. Meyries, Nonlinear elliptic problems with dynamical boundary conditions of reactive and reactive-diffusive type, Proc. Lond. Math. Soc. 108 (2014), 1351-1380.

[33] M. Gao, X.-P.Wang, A gradient stable scheme for a phase field model for the moving contact line problem, J. Comput. Phys. 231 (2012), 1372-1386.

[34] G. Gilardi, A. Miranville, G. Schimperna, On the Cahn-Hilliard equation with irregular potentials and dynamic boundary conditions, Commun. Pure Appl. Anal. 8 (2009), 881-912.

[35] M. Grasselli, A. Miranville, G. Schimperna, The Caginalp phase-field system with coupled dynamic boundary conditions and singular potentials, Discrete Contin. Dyn. Syst. 28 (2010), 67-98.

[36] M.E. Gurtin, D. Polignone, J. Viñals, Two-phase binary fluids and immiscible fluids described by an order parameter, Math. Models Meth. Appl. Sci. 6 (1996), 8-15.

[37] M. Heida, J. Málek, K.R. Rajagopal, On the development and generalizations of Cahn-Hilliard equations within a thermodynamic framework, Z. Angew. Math. Phys. 63 (2012), 145-169.

[38] M. Heida, On the derivation of thermodynamically consistent boundary conditions for the Cahn-HilliardNavier-Stokes system, Internat. J. Engrg. Sci. 62 (2013), 126-156.

[39] M. Hintermüller, D. Wegner, Optimal Control of a Semidiscrete Cahn-Hilliard-Navier-Stokes System, SIAM J. Control Optim. 52 (2014), 747-772.

[40] P.C. Hohenberg, B.I. Halperin, Theory of dynamic critical phenomena, Rev. Mod. Phys. 49 (1977), $435-479$.

[41] D. Jacqmin, Calculation of two-phase Navier-Stokes flows using phase-field modelling, J. Comp. Phys. 155 (1999), 96-127.

[42] D. Jasnow, J. Viñals, Coarse-grained description of thermo-capillary flow, Phys. Fluids 8 (1996), 660-669.

[43] D. Kay, V. Styles, R. Welford, Finite element approximation of a Cahn-Hilliard-Navier-Stokes system, Interfaces Free Bound. 10 (2008), 15-43.

[44] R. Kenzler, F. Eurich, P. Maass, B. Rinn, J. Schropp, E. Bohl, W. Dieterich, Phase separation in confined geometries: solving the Cahn-Hilliard equation with generic boundary conditions, Comput. Phys. Commun. 133 (2001) 139-157.

[45] J. Kim, K. Kang, J. Lowengrub, Conservative multigrid methods for Cahn-Hilliard fluids, J. Comput. Phys. 193 (2004), 511-543.

[46] J.S. Kim, Phase-field models for multi-component fluid flows, Commun. Comput. Phys. 12 (2012), 613-661.

[47] A.G. Lamorgese, D. Molin, R. Mauri, Phase field approach to multiphase flow modeling, Milan J. Math. 79 (2011), 597-642.

[48] J.-L. Lions, Quelques méthodes de résolution des problèmes aux limites non linéaires, Paris: Dunod, 1969.

[49] C. Liu, J. Shen, A phase field model for the mixture of two incompressible fluids and its approximation by a Fourier-spectral method, Phys. D 179 (2003), 211-228.

[50] J. Lowengrub, L. Truskinovsky, Quasi-incompressible Cahn-Hilliard fluids and topological transitions, R. Soc. Lond. Proc. Ser. A Math. Phys. Eng. Sci. 454 (1998), 2617-2654.

[51] D. Maxwell, Initial Data for Black Holes and Rough Spacetimes, PhD thesis, University of Washington, 2004.

[52] J.C. Maxwell, On stresses in rarified gases arising from inequalities of temperature, Philos. Trans. R. Soc. (1879), 704-712.

[53] A. Miranville and S. Zelik, Exponential attractors for the Cahn-Hilliard equation with dynamic boundary conditions, Math. Models Appl. Sci. 28 (2005), 709-735.

[54] H. K. Moffatt, Viscous and resistive eddies near a sharp corner, J. Fluid Mech. 18 (1964), 1-18.

[55] C.L. Navier, Sur les lois de l'équilibre et du mouvement des corps élastiques, Mem. Acad. R. Sci. Inst. France 6 (1827), 369.

[56] L. Onsager, Reciprocal relations in irreversible processes, I. Phys. Rev. 37 (1931), 405-426.

[57] L. Onsager, Reciprocal relations in irreversible processes. II. Phys. Rev. 38 (1931), 2265-2279.

[58] T.-Z. Qian, X. Wang, P. Sheng, Molecular scale contact line hydrodynamics of immiscible flows, Phys. Rev. E 68 (2003), 016306.

[59] T.-Z. Qian, X. Wang, P. Sheng, A variational approach to moving contact line hydrodynamics, J. Fluid Mech. 564 (2006), 333-360.

[60] P. Rybka and K.-H. Hoffmann, Convergence of solutions to Cahn-Hilliard equation, Commun. Partial Differ. Equ. 24 (1999), 1055-1077.

[61] R. Ruiz, D.R. Nelson, Turbulence in binary fluid mixtures, Phys. Rev. A 23 (1981), 3224-3246.

[62] J. Shen, X. Yang, Energy Stable Schemes for Cahn-Hilliard Phase-Field Model of Two-Phase Incompressible Flows, Chin. Ann. Math. Ser. B 31 (2010), 743-758.

[63] J. Shen, X. Yang, H. Yu, Efficient energy stable numerical schemes for a phase field moving contact line model, J. Comput. Phys. 284 (2015), 617-630. 
[64] J. Simon, Compact sets in the space $L^{p}(0, T ; B)$, Ann. Mat. Pura Appl. (4) 146 (1987), 65-96.

[65] E.D. Siggia, Late stages of spinodal decomposition in binary mixtures, Phys. Rev. A 20 (1979), 595-605.

[66] J. Sprekels, H. Wu, A note on parabolic equation with nonlinear dynamical boundary condition, Nonlinear Anal. 72 (2010), 3028-3048.

[67] V.N. Starovoitov, The dynamics of a two-component fluid in the presence of capillary forces, Math. Notes 62 (1997), 244-254.

[68] Z. Tan, K.M. Lim, B.C. Khoo, An adaptive mesh redistribution method for the incompressible mixture flows using phase-field model, J. Comput. Phys. 225 (2007), 1137-1158.

[69] M.E. Taylor, Partial differential equations I, Basic theory, second edition, Applied Mathematical Sciences 115, Springer, New York, 2011.

[70] M.E. Taylor, Partial differential equations III, Nonlinear equations, second edition, Applied Mathematical Sciences 117, Springer, New York, 2011.

[71] R. Temam, Infinite-dimensional dynamical systems in mechanics and physics, Applied Mathematical Sciences, 68, Springer-Verlag, New York, 1997.

[72] Y. Shi, K. Bao, X.-P. Wang, 3D adaptive finite element method for a phase field model for the moving contact line problems, Inverse Probl. Imaging 7 (2013), 947-959.

[73] X.-P. Wang and Y.-G. Wang, The sharp interface limit of a phase field model for moving contact line problem, Methods Appl. Anal. 14 (2007), 285-292.

[74] $\mathrm{H}$. Wu and S. Zheng, Convergence to equilibrium for the Cahn-Hilliard equation with dynamic boundary conditions J. Differential Equations 204 (2004), 511-531.

[75] P. Yue, C. Zhou, J.J. Feng, Sharp-interface limit of the Cahn-Hilliard model for moving contact lines, J. Fluid Mech. 645 (2010), 279-294.

[76] L. Zhao, H. Wu, H. Huang, Convergence to equilibrium for a phase-field model for the mixture of two viscous incompressible fluids, Commun. Math. Sci. 7 (2009), 939-962.

[77] S. Zheng, Nonlinear Evolution Equations, Pitman Ser. Monogr. Survey in Pure and Appl. Math. 133, Chapman \& Hall/CRC, Boca Raton, FL, 2004.

[78] Y. Zhou, J. Fan, The vanishing viscosity limit for a 2D Cahn-Hilliard-Navier-Stokes system with a slip boundary condition, Nonlinear Anal. Real World Appl. 14 (2013), 1130-1134.

Department of Mathematics \& Statistics, Florida International University, Miami, FL 33199, USA

E-mail address: cgal@fiu.edu

Dipartimento di Matematica, Politecnico di Milano, 20133 Milano, Italy

E-mail address: maurizio.grasselli@polimi.it

Laboratoire de Mathématiques et Applications, Université de Poitiers, Boulevard Marie et Pierre Curie - Téléport 2, 86962 Chasseneuil Futuroscope Cedex, France

E-mail address: alain.miranville@math.univ-poitiers.fr 